\title{
Evaluation of Empirical Atmospheric Diffusion Data
}

Prepared by T. W. Horst, J. C. Doran, P. W. Nickola

Battelle Pacific Northwest Laboratories

Prepared for

U. S. Nuclear Regulatory

Commission 


\section{NOTICE}

This report was prepared as an account of work sponsored by an agency of the United States Government. Neither the United States Government nor any agency thereof, or any of their employees, makes any warranty, expressed or implied, or assumes any legal liability or responsibility for any third party's use, or the results of such use, of any information, apparatus product or process disclosed in this report, or represents that its use by such third party would not infringe privately owned rights.

and 
DISCLAIMER

This report was prepared as an account of work sponsored by an agency of the United States Government. Neither the United States Government nor any agency Thereof, nor any of their employees, makes any warranty, express or implied, or assumes any legal liability or responsibility for the accuracy, completeness, or usefulness of any information, apparatus, product, or process disclosed, or represents that its use would not infringe privately owned rights. Reference herein to any specific commercial product, process, or service by trade name, trademark, manufacturer, or otherwise does not necessarily constitute or imply its endorsement, recommendation, or favoring by the United States Government or any agency thereof. The views and opinions of authors expressed herein do not necessarily state or reflect those of the United States Government or any agency thereof. 


\section{DISCLAIMER}

Portions of this document may be illegible in electronic image products. Images are produced from the best available original document. 
NUREG/CR-0798

PNL-2599

$\mathrm{R} 6$, RB

EVALUATION OF EMPIRICAL

ATMOSPHERIC DIFFUSION DATA

T. W. Horst J. C. Doran

P. W. Nickola

First Draft: February 1978

Final Draft: April 1979

Date Published: Octo ber 1979

Atmospheric Sciences Department

Battelle Pacific Northwest Laboratories

Richland, Washington 99352

\footnotetext{
Prepared for

Division of Reactor Safety Research

U.S. Nuclear Regulatory Commission

Washington, D.C. 20555

Under Contract No. EY-76-C-06-1830

NRC FIN No. B2086
} 


\section{ABSTRACT}

A study has been made of atmospheric diffusion over level, homogeneous terrain of contaminants released from non-buoyant point sources up to $100 \mathrm{~m}$ in height. Current theories of diffusion are compared to empirical diffusion data, and specific dispersion estimation techniques are recommended which can be implemented with the on-site meteorological instrumentation required by the Nuclear Regulatory Commission. A comparison of both the recommended diffusion model and the NRC diffusion model with the empirical data demonstrates that the predictions of the recommended model have both smaller scatter and less bias, particularly for groundlevel sources.

For ground-level sources, the Gaussian plume model is an adequate descriptor of the ground-level distribution of contamination. The lateral spread of the plume $\sigma_{y}$ is best estimated from $S=\sigma_{y} / x \sigma_{\theta}$ as a function of downwind distance $x$. The dependence of $S$ on sampling time, averaging time, wind speed, atmospheric stability and surface roughness is discussed, and values of $S$ for sampling times of $30 \mathrm{~min}$ and $60 \mathrm{~min}$ are recommended out to $x=10 \mathrm{~km}$. The vertical spread $\sigma_{z}$ is predicted well by Lagrangian similarity theory if the contaminant is assumed to have a vertical eddy diffusivity equal to that of heat. The effective transport wind speed is found at the height $0.5 \sigma_{z}$ and hence is a function of downwind distance.

For elevated releases, $\sigma_{y}$ at ground level can also be estimated from the recommended function $S$, provided $\sigma_{\theta}$ is measured at ground level. The vertical distribution is Gaussian only very close to the source, $\sigma_{z} \approx 0.7 h$ where $h$ is the source height. Under neutral stability conditions, the associated values of $\sigma_{z}$ are similar to those predicted for groundlevel sources, but for strongly stable conditions they are greater than those for ground-level sources. Based on empirical data for neutral to stable atmospheric conditions, the ratios of ground-level concentrations due to releases from $2 \mathrm{~m}, 26 \mathrm{~m}, 56 \mathrm{~m}$ and $111 \mathrm{~m}$ are displayed as a function of downwind distance. 
-

- 
Page

LIST OF FIGURES

viii

LIST OF TABLES

xiii

CHAPTER

1. INTRODUCTION

1.1 Background

1.2 Data Base

1.3 Theoretical Approach

2. SUMMARY

2.1 Lateral Dispersion

2.2 Vertical Dispersion

2.3 The Net Effect of Increased Source Height on Ground-Level Concentrations

2.4 Comparison of Recommended Model to NRC Model

2.5 Limitations of Available Data and Models

3. DATA BASE

3.1 Prairie Grass

3.2 National Reactor Test Station (NRTS)

3.3 Green Glow and 30-Series

3.4 Hanford 67-Series

\subsubsection{Lateral Dispersion Analysis}

\subsubsection{Vertical Dispersion Analysis}

3.5 Ocean Breeze and Dry Gulch

3.6 Mountain Iron

3.7 Jülich

3.8 Karlsruhe

4. LATERAL DISPERSION 23

4.1 Introduction 23

4.2 Ground-Level Sources 24

4.2.1 Basic Concepts $\quad 24$ 
4.2.2 Sampling Time and Wind Speed Effects 26

4.2.3 Sample Spectra 31

4.3 Effects of Release Height and Terrain Roughness 38

4.3.1 Effects of Release Height 38

4.3.2 Effects of Terrain Roughness 41

4.3.3 Elevated Releases in Rough Terrain 45

4.4 Conclusions 45

5. VERTICAL DISPERSION 48

5.1 Introduction 48

5.2 Ground-Level Sources 49

5.2.1 Background 49

5.2.2 Prediction of the Ground-Level Concentration 50

5.2.3 Comparison with Prairie Grass Observations 55

5.2.4 Comparison with NRTS and Hanford Observations 64

5.2.5 Discussion and Application 72

5.3 Elevated Sources: Hanford 67-Series 74

5.3.1 Concentration Profiles from Tower Data 74

5.3.2 $\sigma_{z}$ from Crosswind-Integrated Concentrations $\quad 79$

5.3.3 Comparison with Ground-Level Source Behavior 80

5.4 Elevated Sources: Other Data 85

5.5 Conclusions 88

6. THE NET EFFECT OF INCREASED SOURCE HEIGHT ON GROUND- 90

LEVEL CONCENTRATIONS

6.1 Introduction 90

6.2 Experimental Approach 90

6.3 Data and Discussion 95

6.4 Conclusions 106

7. COMPARISON OF RECOMMENDED MODEL TO NRC MODEL 107

7.1 Recommended Diffusion Moder 107

7.2 NRC Diffusion Mode1 109 
7.3 Comparison of Model Predictions

109

7.3.1 Lateral Diffusion

109

7.3.2 Vertical Diffusion

113

7.4 Conclusions

123

REFERENCES

125

APPENDIXES

A. Computed Vertical Diffusion Data for Prairie Grass 
Figure

Page

4.1 Variation of $S=\sigma_{y} / x \sigma_{\theta}$ with distance as determined from several field programs.

4.2 Variation of $n F(n)$ (solid line) with frequency. Broken 1 ines show effects of high and low pass filters. (cf. text)

4.3 Variation of $S=\sigma_{y} / x \sigma_{\theta}$ with distance for two wind speed 32 classes. Data are taken from Project Prairie Grass.

4.4 Sample spectra for stable and unstable conditions. spectra are not normalized.

4.5 Variation of $S=\sigma_{y} / x \sigma_{\theta}$ with distance. Values are computed from sample spectra for two values of sampling time $\tau$.

4.6 Variation of $S=\sigma_{y} / x \sigma_{\theta}$ with distance for stable (solid line) and unstable (dashed line) conditions.

4.7 Variation of $S=\sigma_{y} / x \sigma_{\theta}$ with distance for three release 39 heights, Hanford 67-Series. $\sigma_{\theta}$ was measured at $1.5 \mathrm{~m}$ elevation.

4.8 Variation of $S=\sigma_{y} / X_{\theta}$ with distance for three release heights, Hanford 67-Series. $\sigma_{\theta}$ was measured at release heights.

4.9 Variation of $S=\sigma_{y} / x_{\theta}$ with distance for field programs over terrain with different roughnesses.

4.10 Comparison of curve for $S=\sigma_{y} / x \sigma_{\theta}$ recommended for flat 44 homogeneous terrain with Mountain Iron data.

4.11 Variation of $S=\sigma_{y} / x \sigma_{\theta}$ with distance for three field programs with release heights of $\sim 50 \mathrm{~m}$, but different roughnesses. $\sigma_{\theta}$ was measured at release height. 
5.1 Predicted CWIC at ground-level as a function of downwind distance for various stability conditions.

5.2 Predicted and measured CWIC $50 \mathrm{~m}$ downwind of the source as a function of atmospheric stability, Project Prairie Grass.

5.3 Predicted and measured CWIC $100 \mathrm{~m}$ downwind of the source as a function of atmospheric stability, Project Prairie Grass.

5.4 Predicted and measured CWIC $200 \mathrm{~m}$ downwind of the source as a function of atmospheric stability, Project Prairie Grass.

5.5 Predicted and measured CWIC $400 \mathrm{~m}$ downwind of the source as a function of atmospheric stability, Project Prairie Grass.

5.6 Predicted and measured CWIC $800 \mathrm{~m}$ downwind of the source as a function of atmospheric stability, Project Prairie Grass.

5.7 Predicted versus measured CWIC for Project Prairie Grass.

5.8 Predicted and measured CWIC $200 \mathrm{~m}$ downwind of the source as a function of atmospheric stability, NRTS.

5.9 Predicted and measured CWIC $800 \mathrm{~m}$ downwind of the source as a function of atmospheric stability, NRTS.

5.10 Predicted and measured CWIC $3200 \mathrm{~m}$ downwind of the source as a function of atmospheric stability, NRTS.

$5.11 \sigma_{z} 200 \mathrm{~m}$ downwind of the source as a function of a tmospheric stability, Green Glow-30 Series. 
Figure

5.12 Predicted and measured ratio of CWIC at $800 \mathrm{~m}$ to that at $200 \mathrm{~m}$ as a function of atmospheric stability, Green Glow-30 Series.

5.13 Predicted and measured ratio of CWIC at $3.2 \mathrm{~km}$ to that at $200 \mathrm{~m}$ as a function of atmospheric stability, Green Glow-30 series.

5.14 Predicted and measured ratio of CWIC at $12.8 \mathrm{~km}$ to that 71 at $200 \mathrm{~m}$ as a function of atmospheric stability, Greeri Glow-30 Series.

5.15 Comparison of measured concentration profile and best fit 76 Gaussian at $200 \mathrm{~m}$ downwind for $26 \mathrm{~m}$ release, Test $\mathrm{V} 6$.

5.16 Comparison of measured concentration profile and best fit Gaussian at $200 \mathrm{~m}$ downwind for $26 \mathrm{~m}$ release, Test V2.

5.17 Comparison of measured concentration profile and best 78 fit Gaussian at $800 \mathrm{~m}$ downwind for $26 \mathrm{~m}$ release, Test V6.

5.18 Comparison of $\sigma_{z}$ at $200 \mathrm{~m}$ downwind for $26 \mathrm{~m}$ releases with Lagrangian similarity predictions for groundlevel releases.

5.19 Comparison of $\sigma_{z}$ at $400 \mathrm{~m}$ downwind for $26 \mathrm{~m}$ releases with Lagrangian similarity predictions for ground-level releases.

5.20 Comparison of $\sigma_{z}$ at $400 \mathrm{~m}$ downwind for $56 \mathrm{~m}$ releases with 83 Lagrangian similarity prediction for ground-level releases.

6.1 The observed ratio of fluorescein to zinc sulfide 92 tracers following release from a common source.

6.2 Normalized CWIC values observed during field test 94 U78. $\mathrm{h}$ indicates tracer release height.

6.3 Normalized CWIC values observed during field test U79. $h$ indicates tracer release height. 
6.4 Ratio of normalized CWIC observed at $26 \mathrm{~m}$ to that 98 observed at $2 \mathrm{~m}$. Bold curves are observed values; lighter curves are modeled.

6.5 Ratio of normalized CWIC observed at $56 \mathrm{~m}$ to that observed at $26 \mathrm{~m}$. Bold curves are observed values; lighter curves are modeled.

6.6 Ratio of normalized CWIC observed at $111 \mathrm{~m}$ to that observed at $56 \mathrm{~m}$. Bold curves are observed values; 1 ighter curves are modeled.

6.7 Ratio of normalized CWIC at $56 \mathrm{~m}$ to that at $2 \mathrm{~m}$ deduced from observed ratios.

6.8 Ratio of normalized CWIC at $111 \mathrm{~m}$ to that at $2 \mathrm{~m}$ deduced 104 from observed ratios.

6.9 Ratio of normalized CWIC at $111 \mathrm{~m}$ to that at $26 \mathrm{~m}$ deduced 105 from observed ratios.

7.1 Predicted versus measured $\sigma_{y}$ for ground-level releases, $\sigma_{y}=S \times \sigma_{\theta}$.

7.2 Predicted versus measured $\sigma_{y}$ for ground-level releases, NRC $\Delta T / \Delta Z$ model.

7.3 Predicted versus measured $\sigma_{y}$ for ground-level releases, NRC $\sigma_{\theta}$ model.

7.4 Predicted versus measured $\sigma_{y}$ for $26 \mathrm{~m}$ releases, $\sigma_{y}=$ $S \times \sigma_{\theta}$ (ground level).

7.5 Predicted versus measured $\sigma_{y}$ for $26 \mathrm{~m}$ releases, NRC $\sigma_{\theta}$ model (ground-level $\sigma_{\theta}$ ).

7.6 Predicted versus measured $\sigma_{y}$ for $26 \mathrm{~m}$ releases, NRC $\sigma_{\theta}$ model (release-height $\sigma_{\theta}$ ). 
7.7 Predicted versus measured CWIC for Prairie Grass, NRC $\Delta T / \Delta \mathbf{Z}$ model.

7.8 Predicted versus measured CWIC for Prairie Grass, NRC $\sigma_{\theta}$ model.

7.9 Predicted versus measured CWIC for NRTS, Lagrangian similarity model.

7.10 Predicted versus measured CWIC for NRTS, NRC $\sigma_{\theta}$ model. 


\section{LIST OF TABLES}

Table

$\underline{\text { Page }}$

3.1 Hanford 67-Series tests analyzed for lateral

16 dispersion characteristics

$4.1 \quad \sigma_{y} / x_{\theta}$ for ground-level releases 27

4.2 Values of $S$ from Hanford 67-Series 41

4.3 Recommended values of $S=\sigma_{y} / x \sigma_{\theta}$ for $\quad 47$ $t=5$ seconds

5.1 Distribution of the fractional error 62

$5.2 \sigma_{z}$ values at $x=200 \mathrm{~m}$ for $26 \mathrm{~m}$ releases 75

$5.3 \quad \sigma_{z}$ at $400 \mathrm{~m}$ from crosswind-integrated 84 concentrations

5.4 Coefficients and exponents of power laws 87 for $\sigma_{y}$ and $\sigma_{z}$

6.1 Source height, wind speed and stability 97 for dual releases

7.1 NRC Classification of Atmospheric Stability 110

A Computed Vertical Diffusion Data for Prairie Grass 131 
-

- 


\section{ACKNOWLEDGMENTS}

This research was done by the Atmospheric Sciences Department of Battelle, Pacific Northwest Laboratories, for the Division of Reactor Safety Research, U.S. Nuclear Regulatory Commission. NRC technical contract monitor was R. F. Abbey, Jr. of the Site Safety Research Branch.

The authors would like to thank H. E. Cramer, C. E. Elderkin, D. A. Haugen, C. L. Simpson, M. G. Verholek and K. J. Vogt for their help in the collection and interpretation of the diffusion data.

J. A. Bates and P. J. Liddell assisted in the data analysis. 


\section{INTRODUCTION}

\subsection{BACKGROUND}

The atmospheric dispersion of pollutants over relatively level, homogeneous terrain is most commonly predicted by means of the Gaussian plume model, with the parameters $\sigma_{y}$ and $\sigma_{z}$ quantifying the lateral and vertical dimensions of the contaminant plume. The dispersion parameters are estimated from standard sets of curves of $\sigma_{y}$ and $\sigma_{z}$ versus downwind distance $x$, which are based largely on empirical diffusion studies (Gifford, 1976). The most widely used of these sets is probably the socalled Pasquill-Gifford-Turner curves, which present separate curves for each of six atmospheric stability categories defined by observed values of the mean wind speed, solar insolation during daytime hours, and cloudiness at night.

In addition to atmospheric stability and downwind distance, the dispersion parameters also depend on such quantities as the surface roughness, source height, sampling or release time, etc. The Pasquill curves correspond to only one combination of these variables, however, and direct observations of dispersion for all combinations would be prohibitively expensive. Thus theoretical descriptions and models of the diffusion process are required to rationally apply the available data to different circumstances.

A number of diffusion field studies have been carried out since the publication of the Pasquill curves in 1961. These are listed in the following section and described in detail in Chapter 3. Concurrentiy, major advances have been made in the understanding of the turbulent structure of the planetary boundary layer (Haugen, 1972) and new descriptions of atmospheric diffusion have been proposed (Pasquill, 1974; Haugen, 1975). Combination of these three elements can improve the standard methods of predicting atmospheric diffusion.

It has been the goal of this study to make a comprehensive contribution to this improvement. The available empirical diffusion data have been surveyed and those sets have been selected which contain sufficient highquality diffusion and micrometeorological data to allow a test of state-of- 
the-art diffusion theories. Comparisons have been made between theoretical predictions and data, and further development of the models has been made. Finally, specific dispersion estimation techniques have been recommended which can be implemented with the on-site meteorological instrumentation required by the Nuclear Regulatory Commission.

In order to best satisfy the needs of the NRC this study has been limited to non-buoyant, continuous point sources of contamination up to $100 \mathrm{~m}$ in height and to.downwind transport distances of 10 to $15 \mathrm{~km}$. Fortunately the most comprehensive field studies of diffusion have been made under these conditions. In consideration of the available data and state-of-the-art theory, this study has also been limited primarily to diffusion over relatively level, homogeneous terrain.

\subsection{DATA BASE}

In addition to the above criteria for source characteristics and terrain, field studies were also sought with diffusion data complete enough to allow good estimates of $\sigma_{y}$ and/or $\sigma_{z}$ to be made at a number of downwind distances greater than $100 \mathrm{~m}$. It was also desirable, but not mandatory, that the field tests cover a broad range of atmospheric stabilities. Finally, good supporting micrometeorological measurements, such as $\partial u / \partial z, \partial T / \partial z, \sigma_{\theta}$ and $\sigma_{\phi}$, must have been made.

A surprisingly large number of diffusion field studies have been reported. Unfortunately, all but a small fraction of them do not meet the above criteria. For example, Project Sandstorm (Taylor, 1965) sampled materials from high temperature rocket exhausts. The extensive TVA data (Gartrell et al 1964; Carpenter et al, 1968; Thomas et al, 1970) are for $\mathrm{SO}_{2}$ from buoyant stack releases. The Dallas Tower Studies (MacCready et a1, 1961) and many others sponsored by Dugway Proving Grounds (Shearer and Minott, 1976) used aerial line releases which were initially mixed by entrainment in the wake of the dispersal aircraft or incorporated instantaneous, rather than continuous, releases of tracer. The Victoria Trials (Miller, 1966) and the Shoreline Diffusion Program (Smith and Niemann, 1969) were in inhomogeneous terrain near the seacoast. Many very special purpose experiments have been run 
which generated only small amounts of data, e.g., the University of Michigan ragweed studies (Harrington, 1965). The possibility of a series of singular meteorological/diffusion events restricts use of light wind experiments such as those reported by Van der Hoven (1976). Other studies use data from an experiment or a series of experiments, but do not present or reference publications in which the original data are readily available. Singer and Smith's (1966) results-oriented paper serves as an example of this.

The acceptable diffusion experiments are not a great deal more numerous than those referenced in Meteorology and Atomic Energy-1968 (Slade, 1968). They include Project Prairie Grass (Barad, 1958), the National Reactor Testing Station elevated (Islitzer, 1961) and ground-level (Islitzer and Dumbauld, 1963) releases, Project Greenglow (Barad and Fuquay, 1962) and the Hanford "30" Series (Fucuay, et al, 1964). New data for release heights of $50 \mathrm{~m}$ and $100 \mathrm{~m}$ are available from Jülich (Vogt, 1977a,b) and Karlsruhe (Thomas et a1, 1976a,b) and for releases at 2, 26, 56 and $111 \mathrm{~m}$ from Hanford (Nickola, 1977). In addition these recent sets of elevated release data are for quite different values of the surface roughness. (Since one of the largest new sets of diffusion data appears to be the data collected at Hanford (Nickola, 1977), the NRC has jointly sponsored their publication with the Department of Energy.)

A more complete description of the diffusion data sets used in this study has been compiled in Chapter 3 .

\subsection{THEORETICAL APPROACH}

Atmospheric diffusion is an extremely complex phenomenon, involving as it does both the vagaries of geophysical processes and the ordered randomness of turbulent flows. Existing theoretical descriptions are inevitably idealizations or simplifications which focus on one or another mechanism as the dominant feature of the dispersion process and assume that other, often contradictory, aspects can be ignored. Hence, proper application of the theory requires the isolation of diffusion regimes which satisfy the various assumptions and the selection of the appropriate theoretical description. A single, generally applicable theoretical model does not seem feasible at this time. 
Since vertical gradients of atmospheric variables are generaliy much greater than horizontal gradients, diffusion may be usefully divided into a vertical component and a horizontal or lateral component normal to the mean wind direction. (Diffusion in the downwind direction can in most cases be ignored for a continuous source.) Pasquill (1974) states that Taylor's statistical theory is best used for lateral dispersion, because of the theory's assumption of steady, homogeneous turbulence as the dominant dispersive mechanism. This theory relates the crosswind spread of material from a continuous point source directly to the turbulent fluctuations of the wind, and it is employed in Chapter 4 for discussing the lateral dispersion from both ground-level and elevated sources. Except in the immediate vicinity of an elevated source, statistical theory cannot be applied to vertical dispersion due to the dependence on height of the turbulent properties of the lowest kilometer of the atmosphere.

On the other hand, gradient-transfer theory (or K-theory) cannot be applied to lateral dispersion because the size of the eddies effecting lateral dispersion exceeds the scale of the dispersion itself. K-theory can be used only for vertical dispersion from a ground-level source or at distances far enough downwind from an elevated source that it behaves like a ground-level source. (For practical purposes the latter condition requires that the vertical scale of the dispersion be greater than the source height.) In those cases the presence of the lower boundary 1 imits the effective eddies to those which are equal to or smaller than the vertical spread of the contaminant. Similarity theory can also be employed in this regime and, since it is more general and simpler to use than K-theory, it will be used in Section 5.2 to predict vertical diffusion from a surface source.

Taylor's statistical theory implicitly includes the effects of surface roughness, atmospheric stability and wind speed, as well as the longer-period atmospheric fluctuations which may be peculiar to each site. These same variables are explicit input parameters to the similarity theory description of vertical diffusion. Thus the verification of these theories contributes to the project goal of extending predictive capabilities to a large variety of conditions. 
The remaining topic, vertical dispersion from an elevated source when the vertical scale of dispersion is less than or comparable to source height, is treated in Sections 5.3 and 5.4 and also in Chapter 6 . There the observed values of $\sigma_{z}$ and of the ground-level tracer concentration are discussed and compared, with little reference to an organizing theory. Statistical theory could have been used close to the source, but supporting measurements of the vertical component of turbulence were not made for the Hanford-67 data and are not commonly available when diffusion estimates are required. Computer calculations using second-order closure calculations can also be used to simulate this regime, but this method is quite complex and, rather than being a method to organize empirical data, is itself equivalent to an observational program. 


\section{SUMMARY}

\subsection{LATERAL DISPERSION}

The crosswind distribution of an atmospheric contaminant at ground level is found to be best estimated by $S=\sigma_{y} / x \sigma_{\theta}$ as a function of $x . S$ is close to unity near the source of contamination and decreases slowly with increasing distance to a value of 0.5 or less at $10 \mathrm{~km}$.

Examination of a number of sets of diffusion data shows that the relationship between $S$ and $x$ recommended by Pasquill (1976) is an approximate average for a broad range of conditions, but that a more precise formulation is possible which takes into account the effects of such quantities as sampling time, wind speed and atmospheric stability. Variations in $\mathrm{S}$ for different field programs are shown to be attributable to variations in sampling time $\tau$ and averaging time $t$; at a given downwind distance larger values of $S$ are found to be associated with longer $\tau$ and $t$. This behavior, as well as the dependence of $S$ on wind speed and atmospheric stability, is explained in terms of Taylor's statistical theory of diffusion and the Hay-Pasquill relationship between Eulerian and Lagrangian turbulence.

Values of $S$ as a function of $x$ are recommended in Table 4.3 for sampling times of $30 \mathrm{~min}$ and $60 \mathrm{~min}$ and an averaging time for $\sigma_{\theta}$ of $5 \mathrm{sec}$. The values recommended by Pasquill appear to be appropriate for shorter $\tau$.

Table 4.3 is most suitable for flat, homogeneous terrain and groundlevel releases. It will probably also provide reasonable estimates for ground-level releases in moderately rough terrain. For elevated releases in smooth terrain, $\sigma_{\theta}$ at the release height does not appear to be a reliable predictor of $\sigma_{y}$ at ground level, at least for stable conditions. The ground-level value of $\sigma_{\theta}$ is also not appropriate at small distances downwind of elevated sources, but becomes a good predictor farther dowriwind. The obvious extension of these recommendations for elevated releases in rough terrain cannot be tested due to a lack of experimental data which include measurements of $\sigma_{\theta}$ at the surface. 


\subsection{VERTICAL DISPERSION}

The vertical dispersion has generally been irferred from the crosswindintegrated ground-level concentration (CWIC). Since direct measurements of the vertical distribution of a diffusing contaminant are comparatively scarce, they are used mainly to supplement the CWIC data.

The CWIC due to a ground-level source is predicted from Lagrangian similarity theory and compared to several sets of diffusion data. The data clearly show that the eddy diffusitivity of a passive contaminant corresponds more closely to that of heat than that of momentum, and they also support the assumption that the effect of atmospheric stability on the vertical distribution is to alter the scale of the diffusion without strongly affecting the form of the distribution. The standard Gaussian formula (Eq. 5.16) is recommended for predicting the CWIC from ground-level sources, with $\sigma_{z}$ computed from Lagrangian similarity theory, Eq. 5.5, and u specified at the height $0.5 \sigma z$.

The best agreement between Lagrangian similarity theory and data is found for the Prairie Grass data $\left(z_{0}=0.6 \mathrm{~cm}\right)$ to a dimensionless downwind distance of $x / z_{0}=1 \cdot 3 \cdot 10^{5} .94 \%$ of the predictions are between $1 / 2$ and 2 times the measured values, and the r.m.s. fractional error is equal to 0.33. Data from NRTS and Hanford over rougher terrain $\left(z_{0}=1.5 \mathrm{~cm}\right.$ and $3 \mathrm{~cm}$ ) also support the theory to similar dimensionless distances, but they contain considerably more scatter. There is a tendency for all CWIC data from ground-level sources to fall below the predictions at the larger downwind distances. It is not known whether this is due to deposition of the tracer or limitations of the theory.

Al though data for elevated sources are still relatively sparse, some general observations are made on the basis of the Hanford 67-Series. Close to the source, the vertical distribution is fairly well described by the Gaussian formula with an image source to account for the lower boundary. As $\sigma_{z}$ increases and becomes comparable with the release height $h$, however, this formula provides an increasingly poor description of the plume shape. Thus the use of CWIC and a Gaussian formula to estimate $\sigma_{z}$ is useful only for $\sigma_{z} \approx 0.7 \mathrm{~h}$. 
For near-neutral and slightly stable conditions, the Lagrangian similarity prediction of $\sigma_{z}$ for ground-level sources appears to also be adequate for elevated sources. Under more stable conditions, $\sigma_{z}$ for elevated sources is observed to significantly exceed that predicted for ground-level sources. At $400 \mathrm{~m}$ downwind of the source and under neutral to moderately stable conditions, $\sigma_{z}$ for $56 \mathrm{~m}$ releases is approximately 1.5 times larger than for $26 \mathrm{~m}$ releases.

The Jülich measurements provide data for elevated sources in rougher terrain which are useful for estimating both lateral and vertical dispersion. However, only ground-level concentrations have been measured, and the method of data analysis makes comparison or extrapolation of these results difficult. They are recommended for use only under similar conditions of release height and terrain and only for the computation of ground-level concentrations.

\subsection{THE NET EFFECT OF INCREASED SOURCE HEIGHT ON GROUND-LEVEL CONCENTRATIONS}

The net reduction of the CWIC which results from an increase in source height is displayed in Figs. 6.4-6.9. These figures are based on the Hanford 67-Series data for the simultaneous release of tracers from two elevations. To a downwind distance of $10 \mathrm{~km}$, the reduction is less than that predicted on the basis of NRC Regulatory Guide 1.23 and the conventional Gaussian plume model.

The benefits of greater source height increase with $h$ and decrease with $x$. Conceptualiy, the net reduction at ground level is due to two factors: (1) an increase in the vertical diffusion between source height and the surface, and (2) an increase in the initial dilution due to the higher wind speeds at higher source heights. For an increase of source height from $56 \mathrm{~m}$ to $111 \mathrm{~m}$, the reduction at all distances is observed to exceed the wind speed benefit; for an increase from $26 \mathrm{~m}$ to $56 \mathrm{~m}$, the reduction at distances relatively far from the source is approximately equal to the source-height wind speed ratio; and for an increase from $2 \mathrm{~m}$ to $26 \mathrm{~m}$, there was no reduction beyond about $4 \mathrm{~km}$ downwind of the source. These results are consistent with the intuitive notion that beyond a certain downwind distance, which increases with $h$, source height has little effect on the diffusion. 


\subsection{COMPARISON OF RECOMMENDED MODEL TO NRC MODEL}

A comparison of both the recommended diffusion model and the present NRC diffusion model to data from diffusion experiments demonstrates that improved predictions are possible with the recommended model, particularly for ground-level releases. In most cases the recommended diffusion model reduces both the scatter and the bias in the predicted values of $\sigma_{y}$ and CWIC.

The differences between measured values of $\sigma_{y}$ and CWIC and those predicted by the NRC models appear, in many cases, to be caused by the use of $\sigma_{\theta}$ or $\Delta T / \Delta z$ to determine a stability class. The NRC $\sigma_{\theta}$ and $\Delta T / \Delta z$ stability classes are poorly correlated with $\mathrm{l} / \mathrm{L}$, as well as with each other. The prediction of $\sigma_{y}$ is improved in the recommended model by basing its prediction directly on $\sigma_{\theta}$, without reference to a stability class, and by accounting for differences in sampling and averaging time among the sets of diffusion data. The prediction of ground-lever CWIC is improved by using $1 / L$, and hence both the wind and temperature gradients, to specify the atmospheric stability, by accounting for the effect of surface roughness on $\sigma_{z}$, and by specifying the advecting wind at a height of $0.53 \sigma_{z}$.

\subsection{LIMITATIONS GF AVAILABLE DATA AND MODELS}

The available empirical diffusion data demonstrate that the lateral dispersion over homogeneous terrain can be adequately predicted from local measurements of $\sigma_{\theta}$, and data and statistical theory provide good estimates of the relationship between $\sigma_{y}$ and $x \sigma_{\theta}$. However, there is a serious problem when direct observations of $\sigma_{\theta}$ are lacking. Although estimates of wind speed and atmospheric stability can be based on the generally available snyoptic observations, a sound basis does not presently exist for the prediction of $\sigma_{\theta}$. Thus a remaining research task is the estimation of $\sigma_{\theta}$ from less sophisticated measurements. If this research also deals with the spectral distribution of $\sigma_{\theta}$, additional knowledge may also be gained about the relationship between $\sigma_{y}$ and $x \sigma_{\theta}$. 
Due to a lack of direct observations of the vertical contaminant distribution, vertical dispersion is generally inferred from the CWIC at ground level. The CWIC observations indicate that the vertical dispersion from ground-level sources is predicted quite well by Lagrangian similarity theory. The agreement between data and theory deteriorates with increasing downwind distance, however, and this may be due either to shortcomings in the theoretical description of the diffusion process or may be simply due to loss of the tracer by deposition. The existing observations of vertical dispersion from elevated sources may also be influenced by deposition. This is serious because the relative influences of dispersion and deposition are presentiy not known for the observed CWIC. The potential losses due to deposition need to be estimated for the available diffusion data and, if necessary, corrections need to be applied.

The Hanford 67-Series data indicate that $\sigma_{z}$ for elevated sources is similar to that for ground-level sources under near-neutral conditions and is greater for stable conditions. However, the data are quite limited in number and additional observations are especially needed for elevated sources under unstable conditions. Similarly, data are lacking for dispersion over terrain with a roughness length greater than a few $\mathrm{cm}$. The Jülich data fill part of the gap but are themselves limited by the low spatial density of the dispersion measurements.

Finally, gaps are also apparent in the available theoretical descriptions of diffusion, and these cases require further study. The most obvious case is diffusion from elevated sources out to a distance where $\sigma_{z}$ is comparable to $h$. This case is most complicated under stable conditions, when the vertical structure of the atmosphere is the least homogeneous. Observations suggest that the Gaussian plume model may not always be an adequate description of the vertical distribution in this regime. Similarly for vertical dispersion from ground-level sources, the stable case appears to be the least well modeled. 


\section{DATA BASE}

A description of the data sets used in this study is given below. Many of these have been used in previous analyses, but some are relatively new or may be unknown to researchers in this field. Specific comments are appended which are pertinent to the data analyses found in the following chapters.

\subsection{PRAIRIE GRASS}

Reference: Barad (1958)

Dates: July and August, 1956

Terrain: Flat grass stubble, $5-6 \mathrm{~cm}$ high; $z_{0}=0.6 \mathrm{~cm}$

Sampling Distances: $50,100,200, .400$, and $800 \mathrm{~m}$

Sampling Heights: $1.5 \mathrm{~m} ; 0.5 \mathrm{~m}$ to $17.5 \mathrm{~m}$ on 6 towers on $100 \mathrm{~m}$ arc Number of runs: 70

Tracer: $\mathrm{SO}_{2}$

Source Height: $46 \mathrm{~cm} ; 1.5 \mathrm{~m}$ for runs $63-68$

Release Time: 10 minutes

Stability: 34 lapse, 36 inversion

Meteorological Data: Wind speed, temperature and humidity profiles from $25 \mathrm{~cm}$ to $16 \mathrm{~m}$; rawindsonde; some turbulence measurements

Diffusion Data: Tabulation of individual exposures

The 46 tests used in the horizontal dispersion analysis were those originally chosen by Cramer (1957) in his study of the relationship between $\sigma_{y}$ and $\sigma_{\theta}$. They were characterized by the exceptional quality and completeness of the meteorological and $\mathrm{SO}_{2}$ data associated with them. A number of other tests were rejected for a variety of reasons, such as missing or incomplete wind records. Wind speed and direction data for this portion of the study were measured at an elevation of 2 meters.

For the analysis of the vertical dispersion, the wind speed and temperature profiles between $50 \mathrm{~cm}$ and $8 \mathrm{~m}$ were used to estimate $L$ and $u_{*}$ for each diffusion experiment. Second order polynomials in $\ln z$ were first fit 
to the profiles by a least-squares technique, and the gradient Richardson number was calculated at several heights from the fitted profiles and converted to $z / L$ using

$$
z / L=\phi_{m}^{2} R i / \phi_{h}
$$

and empirical formulas for $\phi_{h}$ and $\phi_{m}$. The friction velocity was then calculated by a least-squares fit of an empirical wind profile function to the measured wind profile, using the value of $L$ previously calculated for a height of $2 \mathrm{~m}$ and a roughness length $z_{\circ}=0.6 \mathrm{~cm}$.

\subsection{NATIONAL REACTOR TEST STATION (NRTS)}

References: Elevated Releases (E): Islitzer (1961)

Ground-Level Releases (GL): Islitzer and Dumbauld (1963)

Terrain: Flat with uniformly-spaced, $50 \mathrm{~cm}$ high sagebrush; $z_{0}=1.5 \mathrm{~cm}$ Sampling Distances: 100, 200, 400, 800, 1600 and $3200 \mathrm{~m}$

Sampling Heights: $\sim 7.5 \mathrm{~m} ; 25 \mathrm{~cm}$ to $30 \mathrm{~m}$ on towers on $400 \mathrm{~m}$ arc (GL)

Number of Runs: 16(E) and 33(GL)

Tracer: Uranine

Source Height: $150 \mathrm{ft}(E)$ and $1 \mathrm{~m}(\mathrm{GL})$

Release Time: $30 \mathrm{~min}(\mathrm{E})$ and $60 \mathrm{~min}$ (GL)

Stability: Unstable (E); 25 lapse and 8 inversion (GL)

Meteorological Data: $u, \sigma_{\theta}, \sigma_{\phi}$ and a vertical temperature difference Diffusion Data: $\sigma_{y}, \sigma_{z}$ and normalized CWIC

The elevated release $\sigma_{y}$ results were not tabulated separately for individual tests; instead, a regression line relating $\sigma_{y}$ (measured at ground level) to $x$ and $\sigma_{\theta}$ (measured at 140 feet) was given. Mean wind values at 140 feet were also given. In the treatment of the observed distribution, deviations from "a fairly smooth curve" were "smoothed out", while missing edges of plumes not encompassed by the sampling arcs were estimated by symmetry. The effects of these procedures on the estimates of the $\sigma_{y}$ values are not known.

The GL releases consist of 14 short-range (SR) tests which measured 
tracer to only $800 \mathrm{~m}$ downwind and 19 long-range (LR) tests which measured to $3200 \mathrm{~m}$. $\sigma_{\theta}$ and $\sigma_{\phi}$ were consistently measured at a height of $4 \mathrm{~m}$, but $u$ was measured at both $4 \mathrm{~m}(\mathrm{SR})$ and $8 \mathrm{~m}(\mathrm{LR})$ and $\Delta T$ was measured between $16 \mathrm{~m}$ and $4 \mathrm{~m}(\mathrm{SR})$ and between $8 \mathrm{~m}$ and $1 \mathrm{~m}$ (LR). The gradient Richardson number, and subsequently $\mathrm{L}$, was estimated from the wind and temperature data. Since these data were not available at several heights, empirical formulas for $\phi_{h}$ and $\phi_{m}$ were used to adjust finite-difference gradients, e. g. $\Delta T / \Delta \ln z$, for diabatic effects. The friction velocity was then calculated from the measured wind speed, using the preceeding estimate of $L$ and $z_{0}=1.5 \mathrm{~cm}$.

\subsection{GREEN GLOW AND 30-SERIES}

References: Barad and Fuquay (1962); Fuquay, et a7 (1964)

Dates: June-August, 1959 (GG) and 1960-1962 (30-S)

Terrain: Flat with $1-2 \mathrm{~m}$ sagebrush; $z_{0}=3 \mathrm{~cm}$

Sampling Distance: $200,800,1600,3200$ and $12,800 \mathrm{~m} ; 25,600 \mathrm{~m}$ (GG only) Sampling Heights: $1.5 \mathrm{~m}$; towers to $27 \mathrm{~m}$ at $200 \mathrm{~m}, 42 \mathrm{~m}$ at $800 \mathrm{~m}$, and to $62 \mathrm{~m}$ at $1600 \mathrm{~m}$ and $3200 \mathrm{~m}$.

Number of Runs: 66 (46 selected as best data)

Tracer: Zinc sulfide

Source Height: $\sim 2 \mathrm{~m}$

Release Time: generally $30 \mathrm{~min}$ or $60 \mathrm{~min}$

Stability: 10 unstable, 36 stable selected

Meteorological Data: Wind and temperature profiles to $400 \mathrm{ft}$

Diffusion Data: Tabulation of individual exposures, $\sigma_{y}$, crosswind-peak exposure

46 tests were selected by Fuquay et al (1964), primarily on the basis that the exposure at the edges of the sampling grid was less than one-tenth of the peak exposure and the lateral distribution was not bimodal or multimodal. For Green Glow, wind speed and temperature profiles from $80 \mathrm{~cm}(\mathrm{u})$ and $60 \mathrm{~cm}(T)$ to $12.2 \mathrm{~m}$ were used to compute $L$ (at a height of $3 \mathrm{~m}$ ) and $u_{*}$ in a manner similar to that used for the Prairie Grass data. For the 30Series, a gradient Richardson number was estimated from wind and temperature 
measurements at $7 \mathrm{ft}$ and $50 \mathrm{ft}$ in a manner similar to that used for the NRTS data, and then converted to $z / L$. The friction velocity was calculated from the $7 \mathrm{ft}$ wind speed, using $z_{0}=3 \mathrm{~cm}$.

\subsection{HANFORD 67-SERIES}

Reference: Nickola (1977)

Dates: 1967-1973

Terrain: Flat with $1-2 \mathrm{~m}$ sagebrush; $\mathrm{z}_{\mathrm{o}}=3 \mathrm{~cm}$

Sampling Distances: $200 \mathrm{~m}$ to $12.8 \mathrm{~km}$

Sampling Heights: $1.5 \mathrm{~m}$; towers to $27 \mathrm{~m}$ and $33 \mathrm{~m}$ at $200 \mathrm{~m}, 42 \mathrm{~m}$ at $800 \mathrm{~m}$, and to $62 \mathrm{~m}$ at $1600 \mathrm{~m}$ and $3200 \mathrm{~m}$

Number of Runs: 103 tracer releases during 54 runs

Tracers: Zinc sulfide, fluorescein (uranine), rhodamine B, krypton-85

Source Heights: $2 \mathrm{~m}, 26 \mathrm{~m}, 56 \mathrm{~m}$, and $111 \mathrm{~m}$

Release Time: $10 \mathrm{~min}-30 \mathrm{~min}$

Stability: neutral to stable

Meteorological Data: Wind speed and temperature profiles to $400 \mathrm{ft}$

Diffusion Data: Tabulation of individual exposures and statistical summaries $\left(\sigma_{y}\right.$, peak exposure, etc. $)$

\subsubsection{Lateral Dispersion Analysis}

For each arc, $\sigma_{y}$ was taken about the local center of mass (c.m.), whose angular position often varied from one arc to another. The $\sigma_{y}$ values finaliy used in this study were derived from the above values, but taken with respect to a common center line determined by averaging the c.m. values for each arc in a given test. Corrections of this nature were usually only a few percent. If large systematic trends in the c.m. were evident, data for the run were not used. Also, if the c.m. of one or two arcs showed significant deviations from the c.m. of the remaining arcs, the "odd" arcs were not included in the analysis. Distributions which showed evidence of two or more distinct, major concentration peaks were also discarded.

This selection of the data was carried out because it was felt that an analysis of $\sigma_{y} / x \sigma_{\theta}$ should be restricted, as much as possible, to cases where 
the meteorology was relative uncomplicated. Even with these precautions, the results exhibited a number of complex relationships, as will be seen in Chapter 4.

In a number of cases the full crosswind extent of the diffusion pattern was not embraced by the diffusion grid, and the distribution was therefore severely truncated. Since this truncation could lead to errors in the determination of $\sigma_{y}$, such cases were rejected for further analysis.

As noted in the data volume, not all of the sampling arcs were concentric about the release points. The effects of this on the values of $\sigma_{y}$ attributed to particular downwind distances are minor, as can be verified by reference to the original data.

Finally, for the Hanford 67-Series and all other tests used in the horizontal analysis, arcs beyond a certain downwind distance were not included in the study. This distance, which varied for each test, was determined by the product of the mean wind velocity $u$ at the release height and the duration of the tracer release or sampling time, $\tau$. The restriction, $x \leq u \tau$, essentially ensures that the characteristics of a plume are analyzed, rather than those of a puff. Table 3.1 lists the arcs analyzed in Chapter 4.

$\sigma_{\theta}$ was determined at a number of elevations and by two different types of instruments. Aerovanes were located on a $400 \mathrm{ft}$ meteorological tower and Beckman-Whitley vanes were installed on a portable mast. The latter type of vane was felt to be more responsive and $\sigma_{\theta}$ was taken from them whenever possible. "Ground-level" readings were made at $1.5 \mathrm{~m}$ (Beckman-Whitley) or $2.1 \mathrm{~m}$ (Aerovane). For a $56 \mathrm{~m}$ release height, $\sigma_{\theta}$ was measured at the $200 \mathrm{ft}$ $(61 \mathrm{~m})$ level of the $400 \mathrm{ft}$ tower. For releases at an elevation of $26 \mathrm{~m}, \sigma_{\theta}$ at "release height" was determined at the $100 \mathrm{ft}(30.5 \mathrm{~m})$ level of the tower. Although some data were available at a level of $24.4 \mathrm{~m}$, the $30.5 \mathrm{~m}$ data were used since the latter were available for all of the runs. In 36 tests when data were taken at both levels, the ratio $\sigma_{\theta}(30.5 \mathrm{~m}) / \sigma_{\theta}(24.4 \mathrm{~m})$ had a mean value of 1.02 and a standard deviation of 0.20 ; hence, the data sets appear to be compatible. 
Table 3.1. Hanford 67-Series Tests Analyzed for Lateral Dispersion Characteristics

Test

Ground Level Releases Arcs Analyzed (m)

C1
C2
C4
C5
D1 (ZnS)
(F1)
U57
U58
U59
U61
U62
U63
U64
U67
U70

\begin{tabular}{|c|c|c|c|c|c|c|c|c}
\hline 200 & 400 & 800 & 1200 & 1600 & 2200 & 3200 & 5000 & 7000 \\
$x$ & & $x$ & & & & & & \\
$x$ & & $x$ & & & & & & \\
$x$ & & $x$ & & & & & & \\
$x$ & & $x$ & & & & & & \\
$x$ & & $x$ & & $x$ & & & & \\
& $x$ & $x$ & $x$ & $x$ & & & & \\
& $x$ & $x$ & $x$ & $x$ & & & & \\
& $x$ & & $x$ & & $x$ & & \\
& & $x$ & $x$ & $x$ & $x$ & $x$ & & \\
& $x$ & $x$ & $x$ & $x$ & $x$ & $x$ & $x$ & $x$ \\
& $x$ & $x$ & $x$ & $x$ & & $x$ & & \\
& $x$ & $x$ & $x$ & $x$ & $x$ & $x$ & & \\
& $x$ & $x$ & $x$ & $x$ & $x$ & $x$ &
\end{tabular}

56 Meter Releases

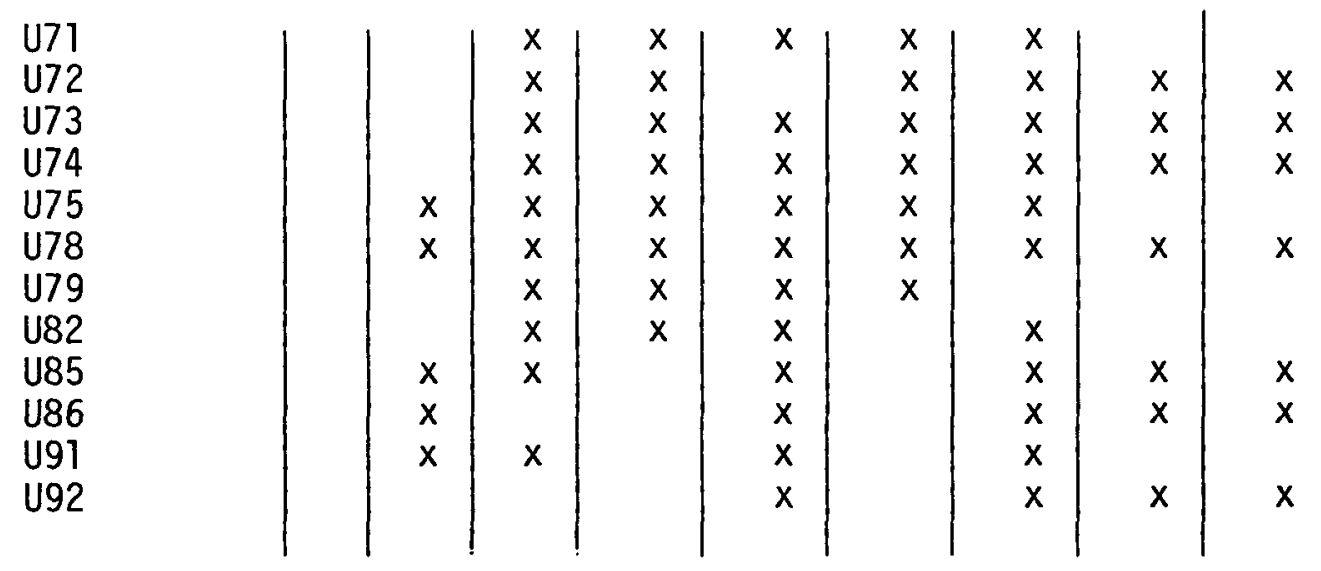


Table 3.1. Continued

$\underline{\text { Test }}$

Arcs Analyzed

U58

U59

U61

U62

U63

U64

U69

U70

U72

U73

U74

U75

U76

U77

U78

U79

U81

U82

U83

V1 (ZnS)

(R)

V2 (R)

V4(ZnS)

V4 (R)

V5 (ZnS)

(R)

V6 (ZnS)

(R)

V8 ( $\mathrm{ZnS})$

\begin{tabular}{|c|c|c|c|c|c|c|c|c|c|}
\hline 200 & 400 & 800 & 1200 & 1600 & 2200 & 3200 & 5000 & 7000 & 1280 \\
\hline & $x$ & $x$ & $x$ & $x$ & & $x$ & & & \\
\hline & & $x$ & $x$ & $x$ & & $x$ & & & \\
\hline & & & $x$ & $x$ & $x$ & $x$ & & & $x$ \\
\hline & & $x$ & $x$ & $x$ & $x$ & $x$ & $x$ & $x$ & \\
\hline & $x$ & $x$ & $x$ & $x$ & & & $x$ & $x$ & \\
\hline & & $x$ & $x$ & $x$ & & & & & \\
\hline & $x$ & $x$ & $x$ & $x$ & & $x$ & $x$ & $x$ & \\
\hline & $x$ & $x$ & $x$ & $x$ & $x$ & $x$ & $x$ & $x$ & $x$ \\
\hline & $x$ & $x$ & $x$ & $x$ & $x$ & $x$ & $x$ & $x$ & \\
\hline & & $x$ & $x$ & $x$ & $x$ & $x$ & $x$ & $x$ & \\
\hline & $x$ & $x$ & $x$ & $x$ & & & $x$ & $x$ & \\
\hline & $x$ & & $x$ & $x$ & $x$ & $x$ & & & \\
\hline & $x$ & $x$ & $x$ & $x$ & $x$ & $x$ & & & \\
\hline & $x$ & $x$ & $x$ & $x$ & & & & & \\
\hline & $x$ & $x$ & $x$ & $x$ & $x$ & $x$ & $x$ & $x$ & $x$ \\
\hline & $x$ & $x$ & $x$ & $x$ & & $x$ & $x$ & $x$ & \\
\hline & & $x$ & . & $x$ & $x$ & 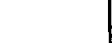 & $x$ & $x$ & \\
\hline & $x$ & $x$ & $x$ & $x$ & $x$ & $x$ & $x$ & $x$ & \\
\hline & $x$ & $x$ & $x$ & $x$ & $x$ & $x$ & $x$ & $x$ & \\
\hline$x$ & $x$ & $x$ & $x$ & $x$ & $x$ & $x$ & & & \\
\hline$x$ & $x$ & $x$ & $x$ & & & & & & \\
\hline$x$ & & $x$ & & $x$ & $x$ & $x$ & & & \\
\hline & & $x$ & & $x$ & & & & & \\
\hline & & $x$ & & $x$ & & & & & \\
\hline$x$ & $x$ & $x$ & $x$ & & & & & & \\
\hline$x$ & $x$ & $x$ & $x$ & $x$ & & & & & \\
\hline$x$ & $x$ & & $x$ & $x$ & & $x$ & & & \\
\hline$x$ & & & $x$ & $x$ & & $x$ & & & \\
\hline$x$ & $x$ & & $x$ & & & & & & \\
\hline
\end{tabular}




\subsubsection{Vertical Dispersion Analysis}

For tests $V 1$ to $V 8$, vertical concentration profiles with a peak value of unity were derived from the available tower measurements. For each tower, the measured concentration profile was normalized by the highest concentration observed at that tower. If the peak of the profile was not obviously embraced by the extent of the tower, the data for that tower were not used. In addition, if the peak concentration at a tower was significantly lower than the largest observed concentration for the arc, e. g., by a factor of 10 or more, the tower data with the low values were also discarded. Normalized concentrations for corresponding levels of all the towers on the same arc were then averaged, and the results normalized to the highest observed average.

For comparison of elevated releases with theoretical predictions, values of $L$ were calculated to quantify the atmospheric stability. In tests $V I$ through $\mathrm{V} 8$, temperatures were measured at heights of 0.91 and $6.1 \mathrm{~m}$, and wind speeds were determined at $0.76,1.5$ and $3.0 \mathrm{~m}$. From these values a gradient Richardson number was computed and converted to a value of $L$ using (3.1) and the expressions for $\phi_{h}$ and $\phi_{m}$ given by Businger et al, (1972). For the remaining tests, temperature measurements were taken at $0.91 \mathrm{~m}$ and $15.2 \mathrm{~m}$. In view of this rather large vertical extent, a bulk Richardson number was determined from these two temperatures and the wind speed at $1.5 \mathrm{~m}$, and $\mathrm{L}$ was computed in the manner described in Section 3.2 for NRTS data. 


\subsection{OCEAN BREEZE AND DRY GULCH}

Reference: Haugen and Fuquay (1963)

Dates: $1961-1962$

Terrain: Rolling, $10 \mathrm{ft}$ to $20 \mathrm{ft}$ sand dunes covered with dense palmetto

( $2 \mathrm{ft}$ to $5 \mathrm{ft}$ ) and brushwood (7 ft to $14 \mathrm{ft}$ ) (OB)

Complex, sloping mesa cut by deep ravines. Vegetation is $1 \mathrm{ft}$ to $2 \mathrm{ft}$ grasses, occasional brush ( $5 \mathrm{ft}$ to $6 \mathrm{ft}$ ) and tree 1 ines

$(50 \mathrm{ft}$ to $80 \mathrm{ft})(\mathrm{DG})$

Sampling Distances: $0.75,1.5$ and 3 miles (OB); 2301 and $5665 \mathrm{~m}$ (DG-B course); 853,1500 and $4715 \mathrm{~m}$ (DG-D course)

Sampling Heights: $15 \mathrm{ft}$, some at $5 \mathrm{ft}(O B) ; 1.5 \mathrm{~m}$ (DG)

Number of Runs: $76(\mathrm{OB})$ and 109 (DG)

Tracer: Zinc Sulfide

Source Height: $22 \mathrm{~m}$

Release Time: $30 \mathrm{~min}$

Stability: $3 / 4$ moderately unstable, $1 / 10$ very unstable, $1 / 10$ moderately stable

Meteorological Data: $u, \sigma_{\theta}$ at a height of $12 \mathrm{ft} ; \Delta T$ from ground-level to about $50 \mathrm{ft}$

Diffusion Data: Tabulation of individual exposures

It is important to note that there may have been some significant errors introduced by the instruments used in these tests. Specifically a Belfort Type $M$ vane was used to measure wind directions. Conversations with individuals actually involved with the experiments revealed misgivings about the performance of the vane. There was apparently a tendency for the instrument to flop back and forth over wide angles, a mode of behavior which is shown by motion pictures of the instrumentation. This behavior might have lead to erroneously high values of $\sigma_{\theta}$, and the interpretation of the results in terms of $\sigma_{y} / x \sigma_{\theta}$ must therefore be treated with caution. Further discussion of this point will be found in Section 4.3.2. 


\subsection{MOUNTAIN IRON}

Reference: Hinds and Nickola (1967, 1968)

Dates: September, 1966 to August, 1967

Terrain: Complex -- mountains, canyons, ridges, marine terrace

Sampling Distances: Variable, from $360 \mathrm{~m}$ to $7820 \mathrm{~m}$

Sampling Height: $1.5 \mathrm{~m}$

Number of Runs Analyzed: 11

Tracer: Zinc Sulfide

Source Height: $\sim 2 \mathrm{~m}$

Release Time: $5 \mathrm{~min}$ to $30 \mathrm{~min}$

Stability: generally unstable

Meteorological Data: $u, \sigma_{\theta}$ at $12 \mathrm{ft}$; $\Delta \mathrm{T}$ between $6 \mathrm{ft}$ and $59 \mathrm{ft}$ and between $6 \mathrm{ft}$ and $300 \mathrm{ft}$

As a check on the effects of rough terrain on the ratio of $\sigma_{y} / x \sigma_{\theta}$, selected tests from this series at Vandenberg Air Force Base were studied. These experiments were carried out in an area south of the Dry Gulch tests. The area featured considerably rougher terrain than the Dry Gulch courses, and there were a number of cases in which the air flow was clearly affected by large scale terrain features. In order to study diffusion which did not show such properties, this analysis was therefore confined to those tests with lateral distributions which were approximately Gaussian. These were the "modified cigar" patterns described in Volume II of the report on those experiments. The runs finally studied were tests $3,6,9,11,13,15,19,21,23,25,26,27,50$, and 107. Because of the short tracer release times in many of these, only a relatively small number of measurements actually satisfied the criterion that wind speed multiplied by release time be greater than or equal to the downwind distance. 


\subsection{JÜLICH}

Reference: Vogt et al (1973) and Vogt (1977a, 1977b)

Dates: 1969-1976

Terrain: Woodland and pastures, $z_{0}=40 \mathrm{~cm}$ to $1.8 \mathrm{~m}$

Sampling Distances: $100 \mathrm{~m}$ to $10 \mathrm{~km}$

Sampling Heights: ground-level only at up to 120 locations

Number of Runs: 60

Tracers: $\mathrm{Cu}^{64}$ or $\mathrm{Ho}^{166}$ tagged copper or holmium sulphate aerosol

Source Heights: $50 \mathrm{~m}$ ( 37 runs) and $100 \mathrm{~m}$ ( 29 runs)

Release Time: 60 min

Stability: mostly neutral to unstable

Meteorological Data: Wind profiles to $120 \mathrm{~m} ; \sigma_{\theta}$ and $\sigma_{\phi}$ at $50 \mathrm{~m}$ and $120 \mathrm{~m}$; $\Delta T(120 \mathrm{~m}-20 \mathrm{~m})$; insolation

Diffusion Data: $\sigma_{y}$ and $\sigma_{z}$ for each test expressed as a power function of $x$; individual measurements of $\sigma_{y}$ are available up to test 25

The sampling grid was rather sparse compared to other field programs and a significant effort was devoted to the implementation of data fitting routines to extract values of $\sigma_{y}$ and $\sigma_{z}$. Unfortunately, many of the results are presented in the form of power law parameters which the investigators bel ieve best represent their findings. The original data from which these parameters are derived is not generally available in a form which is compatible with an analysis in terms of $\sigma_{y} / x_{\theta}$.

In some of the earlier tests, $\sigma_{y}$ was estimated from horizontal concentration distributions, and the results were tabulated in Table 19 of Vogt et al (1973). Since only a small number of sampling stations was actually located on a given "arc", some subjective judgment was required in fitting Gaussian curves to these results. 
Fourteen tests, all with $50 \mathrm{~m}$ release heights, were finally chosen for study of the horizontal dispersion. One test (Test 6 ) was rejected because of excessive shifts in the wind direction during the release period, and another (Test 15) was discarded because of extremely large directional wind shear in the vertical.

The results from these and the remaining tests have been analyzed in another fashion by $\operatorname{Vogt}(1977 \mathrm{a}, \mathrm{b})$. Further details are presented in Section 5.4 .

\subsection{KARLSRUHE}

The Karlsruhe experiments (Thomas et al, 1976a,b) involved a series of releases from a height of $100 \mathrm{~m}$. The analysis was based on the assumption of a Gaussian form for the dispersion of material, wherein $\sigma_{y}$ and $\sigma_{z}$ varied as powers of the downwind distance. A non-linear least-squares fit to the data was made to determine $\sigma_{y}$ and $\sigma_{z}$.

For these tests, the actual concentration data is available so that values of $\sigma_{y}$ could presumably also be determined directly by calculation of the second moment of the distribution. However, the number of data points of each "arc" is generally quite small, and the distribution is not well defined. It was not felt that inclusion of these data in the current analysis was justified. 


\section{LATERAL DISPERSION}

\subsection{INTRODUCTION}

Observations indicate that the Gaussian plume model

$$
x(x, y, z)=\frac{Q e^{-y^{2} / 2 \sigma_{y}^{2}}}{2 \pi u \sigma_{y}{ }^{\sigma} z}\left\{\exp \left[-\frac{(z-h)^{2}}{2 \sigma z^{2}}\right]+\exp \left[-\frac{(z+h)^{2}}{2 \sigma z^{2}}\right]\right\}
$$

is a good approximation to diffusion from a continuous source, particularly for horizontal spread and for vertical spread in regions clear of the ground (Pasquil1, 1974). In this chapter the crosswind distribution, characterized by its standard deviation $\sigma_{y}$, is investigated independently of the vertical distribution. This separation of the lateral and vertical dispersion is essential to a fundamental understanding of atmospheric diffusion because each can be effected by different mechanisms.

Values of $\sigma_{y}$ were obtained directly from the ground-level, crosswind distributions of tracer measured during eleven different field programs. (Details of the data anlysis are found in Chapter 3, particulary section 3.4.) Thus only the relative tracer concentrations, and not their absolute values, are meaningful to this analysis. The measured values of $\sigma_{y}$ were studied to determine their dependence on the standard deviation of the horizontal wind direction $\sigma_{\theta}$, downwind distance from the source $x$, wind speed $u$, atmospheric stability, surface roughness and release height. Initial efforts along these 1 ines indicated the extreme complexity which characterizes these relationships, and therefore attempts were made to cast the problem in a simplified form using only a few parameters.

The use of a purely empirical approach, applicable to individual test series, was rejected, since similarities and differences with results from other series are then difficult to explain. For this reason, simple laws relating $\sigma_{y}$ to some power of $x, \sigma_{\theta}$ or some other combination of the two were not used. Analysis of the data in terms of the dimensionless ratio $\sigma_{y} / x \sigma_{\theta}$ appeared most promising. Results of all field studies were therefore cast into this form whenever possible. 


\subsection{GROUND-LEVEL SOURCES}

\subsubsection{Basic Concepts}

The dimensionless ratio

$$
S=\frac{\sigma_{y}}{x \sigma_{\theta}}
$$

has been examined in a number of studies (e.g., Pasquill, 1971; Draxler, 1976), and various empirical relationships for the dependence of $S$ on downwind distance or travel time have been proposed. In general, there is considerable scatter of the experimental data about the "universal" curves which have been suggested, but agreement within about a factor of two or better is usually obtained.

Attempts to derive a theoretical expression for the variation of $S$ with $x$ normally begin with a consideration of statistical analyses of the diffusion process. If $F_{L}(n)$ is the Lagrangian frequency spectrum of the lateral wind component, then one form of Taylor's theorem is (Ogura, 1959; Pasquil1, 1974)

$$
\left[\begin{array}{c}
\sigma_{y}^{2} \\
{ }^{2}
\end{array}\right]_{\tau, T}=\left[\sigma_{v}{ }^{2}\right]_{\infty, 0} T^{2} \int_{0}^{\infty} F_{L}(n)\left(1-\frac{\sin ^{2} \pi n \tau}{(\pi n \tau)^{2}}\right) \frac{\sin ^{2} \pi n T}{(\pi n T)^{2}} d n
$$

where $T$ is the travel time required for the particles to reach the measuring location, $\tau$ is the time over which the dispersion is sampled, and $\sigma_{v}{ }^{2}$ is the variance of the lateral component of the wind velocity. The subscripts on the bracketed $\sigma_{v}{ }^{2}$ refer to an infinitely long sampling time and a vanishingly small averaging time, while the $\tau$ and $T$ subscripts on the bracketed $\sigma_{y}{ }^{2}$ indicate a finite sampling time $\tau$ and an effective averaging time T.

Hay and Pasquill (1959) transformed (4.3) into an Eulerian expression by introducing $\beta$, the ratio of the Lagrangian and Eulerian time scales and writing: 


$$
\left[\sigma_{y}^{2}\right]_{\tau, T}=\left[\sigma_{v}^{2}\right]_{\tau, 0} T^{2} \int_{0}^{\infty} F_{E}(n) \frac{\sin ^{2} \pi n T / \beta}{(\pi n T / \beta)^{2}} d n
$$

where $F_{E}(n)$ is the Eulerian frequency spectrum of the lateral wind component. They argued that (4.4) is valid when $\tau$ is long enough to include the effects of the whole spectrum of turbulence, and that even for smaller $T$ (4.4) is still applicable provided $\tau \geq$ T. If $\sigma_{\theta}$ is sampled over a time $\tau$ but with an averaging time of zero, then $S$ is given more specifically by the ratio $\left[\sigma_{y}\right]_{\tau, T} / x\left[\sigma_{\theta}\right]_{\tau, 0}$. In the limit of large $\tau$, Pasquill (1975) showed that (4.4) leads to an expression for $S$ given by

$$
S=\frac{\left[\sigma_{v}\right]_{\infty}, T / \beta}{\left[\sigma_{v}\right]_{\infty}, 0}
$$

where the relation $\sigma_{v}{ }^{\top} \approx \sigma_{\theta} x$ has been used. For a given turbulent spectrum, (4.5) is a function of $T / t_{L}$ only, where $t_{L}$ is the Lagrangian integral time scale. Alternatively, if the turbulent spectrum is a function of the reduced frequency $f=n z / u$, then one may also show that this ratio is a function of $x$ alone. Such an approach has led Draxler (1976) to propose a set of curves for $S$ as a function of $T$, using representative values of $t_{L}$ to account for different stabilities. Pasquill (1976) has summarized the variation of $S$ with $x$, and suggested values to be used independent of terrain roughness, release height and sampling duration up to one hour.

In the following sections we wish to show that there are, in fact, systematic differences between different sets of experimentally determined values of $S$, as defined in (4.2). Moreover, these differences can be largely explained by a re-examination of the statistical "filtering" which affects $\sigma_{y}$ and $\sigma_{\theta}$ values as determined in various field programs. For example, the restriction $\tau \geq T$ is not sufficient to ensure that $S$ be independent of sampling time. Rather, $S$ will be shown to exhibit a complicated dependence upon $\tau, u, T, x$, and stability. In addition, a spectral model is presented which qualitatively accounts for many of the observed 
characteristics of S. Finally, a revised functional dependence of $S$ on $x$ is suggested.

\subsubsection{Sampling Time and Wind Speed Effects}

In (4.3), $\left[\sigma_{y}{ }^{2}\right]_{\tau, T}$ is the quantity which is generally determined in a dispersion experiment. The variance of wind direction may be measured, along with $\sigma_{y}$, during the duration of the tracer release. This release time is just $\mathrm{\tau}$, as in (4.3) and the wind data are also usually averaged over some period $t$.

If $x \sigma_{\theta} \sim \sigma_{v} T$, then

$x^{2}\left[\sigma_{\theta}^{2}\right]_{\tau, t}=\left[\sigma_{v}{ }^{2}\right]_{\infty, 0} T^{2} \int_{0}^{\infty} F_{E}(n)\left(1-\frac{\sin ^{2} \pi n \tau}{(\pi n \tau)^{2}}\right) \frac{\sin ^{2} \pi n t}{(\pi n t)^{2}} d n$

so that one may define an experimentally determined quantity

$S=\frac{\left[\sigma_{y}\right]_{\tau, T}}{\left.x \mid \sigma_{\theta}\right]_{\tau, t}}=\left\{\frac{\int_{0}^{\infty} F_{L}(n)\left(1-\frac{\sin ^{2} \pi n \tau}{(\pi n \tau)^{2}}\right) \frac{\sin ^{2} \pi n T}{(\pi n T)^{2}} d n}{\int_{0}^{\infty} F_{E}(n)\left(1-\frac{\sin ^{2} \pi n \tau}{(\pi n \tau)^{2}}\right) \frac{\sin ^{2} \pi n t}{(\pi n t)^{2}} d n}\right\}^{1 / 2}$

This is actually the quantity which Draxler (1976) and Pasquill (1975) considered in their analyses of various field programs. It should be noted that the high pass filter functions in the numerator and denominator of (4.7) are the same, since they both contain the same sampling time $\tau$. The low-pass filter functions differ, however, since one contains $t$ and the other $T$. The result is that $S$ must depend on $t, \tau$, and $T$. In Pasquili's study, only two field programs were explicitly treated, each of which had essentially identical sampling times. Draxler incorporated a larger number of tests in his analysis, but did not distinguish between different values of $\tau$, in keeping with the assumptions made in deducing (4.4). 
The results of a number of field studies are shown in Fig. 4.1. The graphs of $S$ as a function of $x$ are seen to order monotonically with sampling time $\tau$. The plotted points for each series of tests are averages measured at the distances indicated. While variations about these averages are generally large enough so that the difference between the means of two series is sometimes less than one standard deviation, the trend is clear. The values suggested by Pasquill (1976) are marked by squares, and are seen to correspond approximately to those associated with sampling times of 10 to 30 minutes and averaging times of 1 to 5 seconds. Table 4.1 gives additional details on the numbers of tests studied and the standard deviations of the averaged values shown in Fig. 4.1

The observed behavior is consistent with that predicted by (4.7), as can be seen from the following qualitative considerations. Fig. 4.2

Table 4.1. $\sigma_{y} / x \sigma_{\theta}$ for Ground-Level Releases

\begin{tabular}{|c|c|c|c|c|c|c|c|c|}
\hline 50 & 100 & 200 & 400 & 800 & 1200 & 1600 & 2200 & 3200 \\
\hline & & 5 & 4 & 15 & 7 & 11 & 4 & 6 \\
\hline & & .76 & .81 & .83 & .58 & .58 & .50 & .53 \\
\hline & & .16 & .14 & .26 & .19 & .20 & .22 & .14 \\
\hline & 23 & 32 & 22 & 27 & & 18 & & 18 \\
\hline & 1.04 & .98 & .92 & .85 & & .77 & & .69 \\
\hline & .16 & .15 & .19 & .16 & & . 17 & & .14 \\
\hline & & 35 & & 34 & & 29 & & 32 \\
\hline & & .94 & & .77 & & .72 & & .67 \\
\hline & & .18 & & .21 & & .24 & & .25 \\
\hline 44 & 46 & 46 & 46 & 46 & & & & \\
\hline .88 & .76 & .68 & .58 & .49 & & & & \\
\hline .14 & .17 & .18 & .20 & .22 & & & & \\
\hline
\end{tabular}




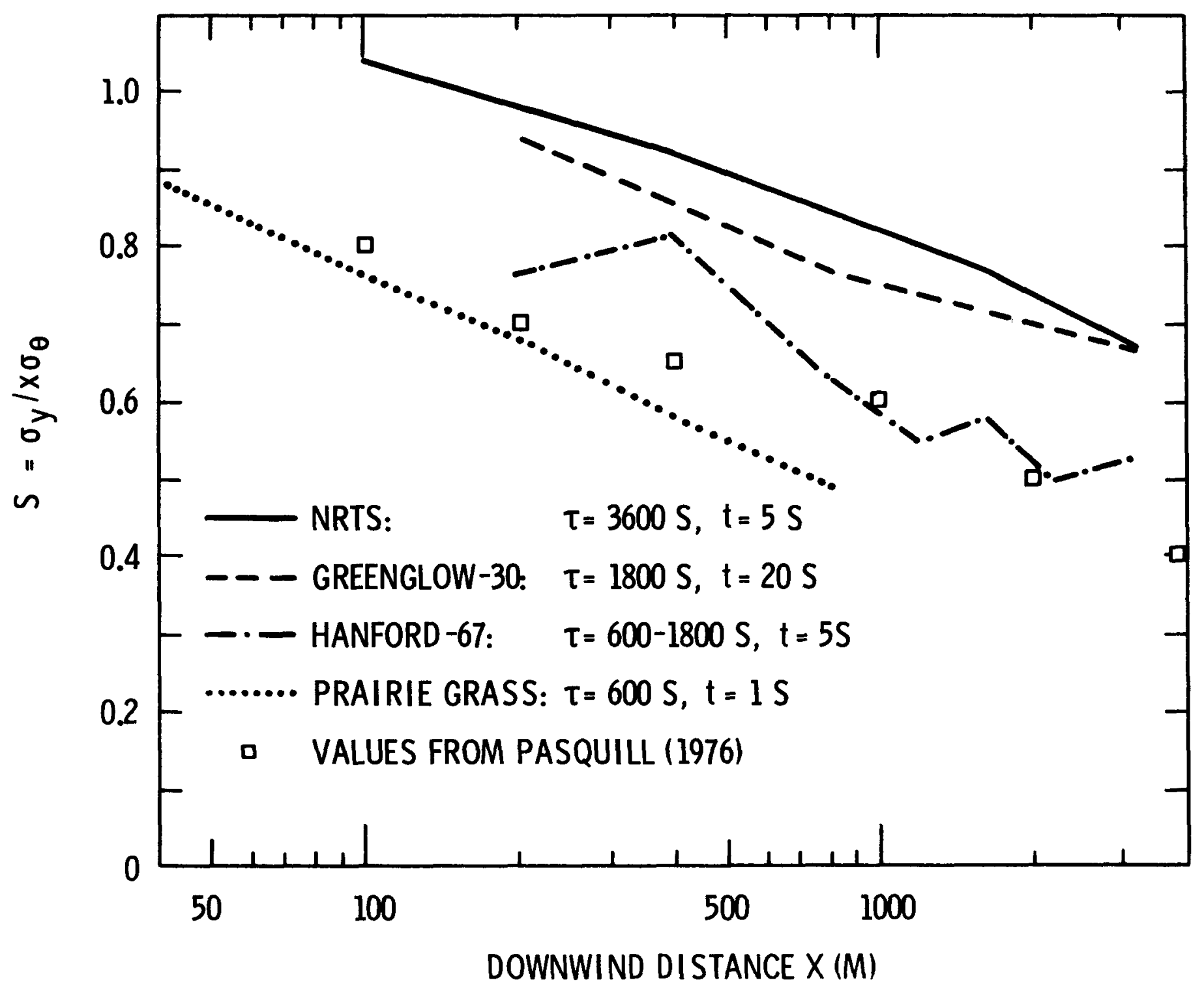

Figure 4.1. Variation of $S=\sigma / x \sigma$ with distance as determined from several field programs. 
กั

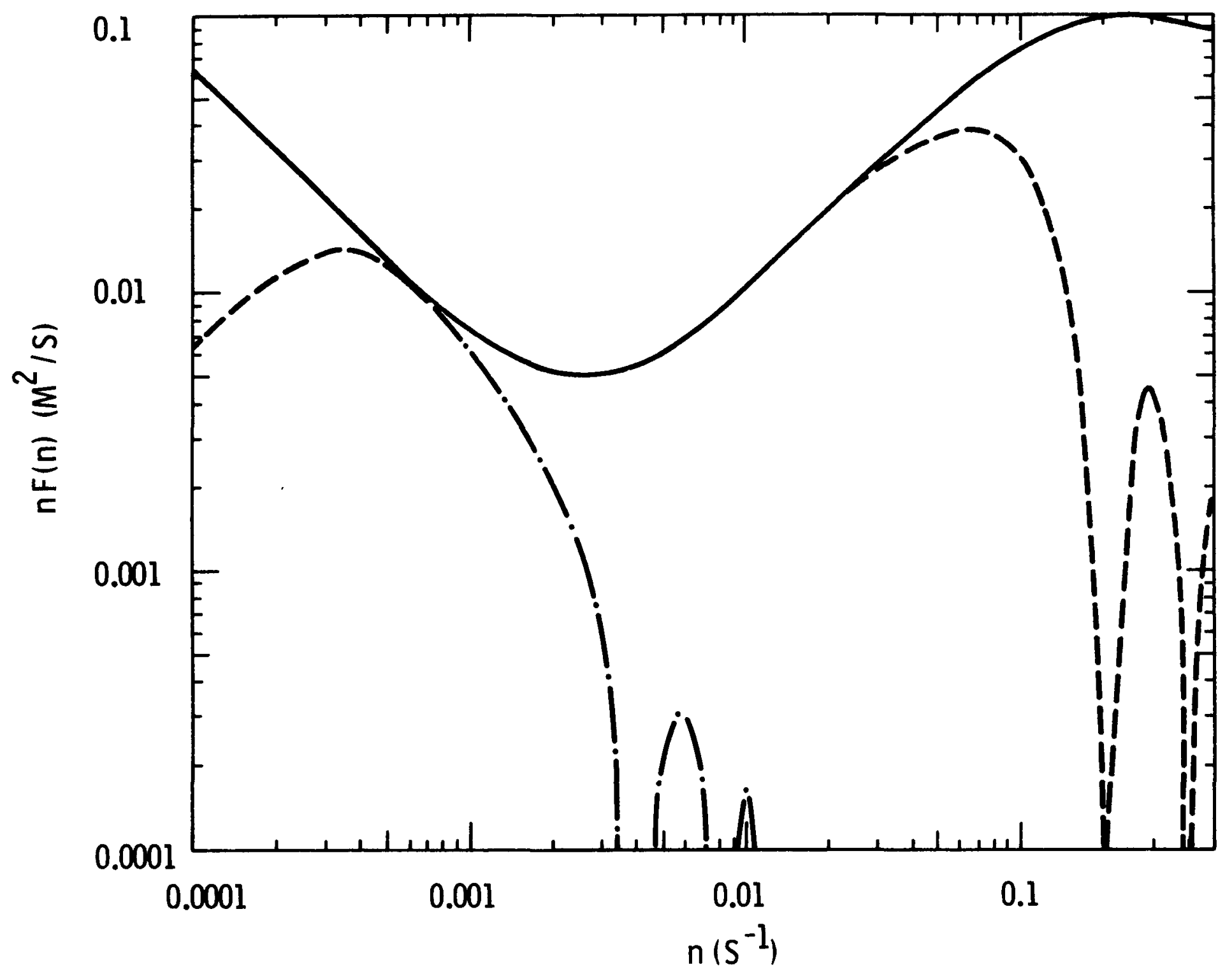

Figure 4.2. Variation of $n F(n)$ (solid line) with frequency. Broken lines show effects of high and low pass filters. 
shows $n F(n)$ (solid line) as a function of $n$, where $F(n)$ is some hypothetical Eulerian or Lagrangian spectrum. The denominator of (4.7) is proportional to the area enclosed by the dashed 1ines; an averaging time of $t=5 \mathrm{sec}$ has been assumed. The numerator is proportional to the area enclosed by the dashed line on the left and the dash-dot line on its right. As $\tau$ increases, the low frequency ends of the filtered spectra move toward the left, increasing the numerator proportionately more than the denominator. If the Lagrangian and Eulerian spectra were identical, this would result in an increase of $S$ with $\tau$, independent of the particular spectrum shape, or averaging time $t$, provided $t$ was shorter than the travel time $T$. The situation is complicated by the fact that these spectra are generally not equal, but a similar dependence of $S$ on $\tau$ can nevertheless be shown to be true over a wide range of assumed spectral behavior. Some examples will be presented later.

Pasquill has noted that ideally $\sigma_{\theta}$ should be determined with $t=0$, whereas in the experiments represented in Fig. 4.1, $t$ varied from 1 to 20 seconds. From (4.7) it is apparent that smaller averaging times will result in smaller values of $S$, other conditions being equal. This probably accounts for part of the large differences between the Prairie Grass results and those obtained from the other field programs. However, the experimental curves do not order monotonically with $t$ as they do with $\tau$, so it is evident that the latter quantity can substantially influence the behavior of $S$ as we11. In the absence of detailed knowledge of the relevant spectra in (4.7), it is impossible to specify which of these contributing factors, $t$ or $\tau$, will have the more important effects. It seems reasonable that both may be significant, and comparisons of results from various field programs should take variations in these quantities into account explicitly. This practice has not been followed in previous analyses.

There is some evidence (e.g., Kaimal et al, 1972) that the Eulerian turbulent velocity spectra are functions of the dimensionless frequency $f=n z / u$. If the same dependence is true for the Lagrangian spectra, then (4.7) may be written in an alternate form, 


$$
S=\left\{\frac{\int_{0}^{\infty} F_{L}(f)\left(1-\frac{\sin ^{2}(\pi f u \tau / z)}{(\pi f u \tau / z)^{2}}\right) \frac{\sin ^{2}(\pi f x / z)}{(\pi f x / z)^{2}} d f}{\int_{0}^{\infty} F_{E}(f)\left(1-\frac{\sin ^{2}(\pi f u \tau / z)}{(\pi f u \tau / z)^{2}}\right) \frac{\sin ^{2}(\pi f u t / z)}{(\pi f u t / z)^{2}} d f}\right\}^{\frac{1}{2}}
$$

where the substitution $T=x / u$ has been made in the numerator. Thus, in addition to $i$ ts dependence on $t$ and $\tau$, $S$ should also vary with the mean wind speed in the same way it varies with $\tau$.

Fig. 4.3 shows the results for 8 tests from Project Prairie Grass with a mean wind speed of $8.0 \mathrm{~m} / \mathrm{s}$ and 6 tests with a mean wind speed of $2.4 \mathrm{~m} / \mathrm{s}$. The wind speed ranges in the two groups were $7.0-9.4 \mathrm{~m} / \mathrm{s}$ and $1.4-2.8 \mathrm{~m} / \mathrm{s}$, respectively. The higher wind speed values are associated with larger values of $S$, in agreement with the behavior predicted by (4.8). Efforts to corroborate this behavior with results from other field programs were inconclusive because of insufficient data or excessive scatter of the data.

\subsubsection{Sample Spectra}

In the discussion thus far, comparisons of measured values of $S$ have been made with the implicit assumption that the spectral behavior in the various cases has been similar. While this may be plausible when comparing ensembles of measurements such as might be collected during an entire field program, it is clear that a more refined characterization of the behavior of $S$ must take these possible differences into account. The spectra depend on the stability and the mean wind speed, but their precise behavior in the frequency regions which contribute most strongly to $S$ is not well defined. For values of $\tau$ and $T$ which are encountered in typical measurements, this corresponds to a range of $f$ from $10^{-4}$ to $10^{-2}$. The situation is particularly unsatisfactory for unstable conditions (Kaimal et al, 1976). Despite these difficulties, it is possible to qualitatively model several features of the behavior of $S$, in addition to the dependence on $t$ and $\tau$ already discussed.

Two sample spectra were constructed, one corresponding to slightly stable conditions and one to slightly unstable conditions. Several assumptions 


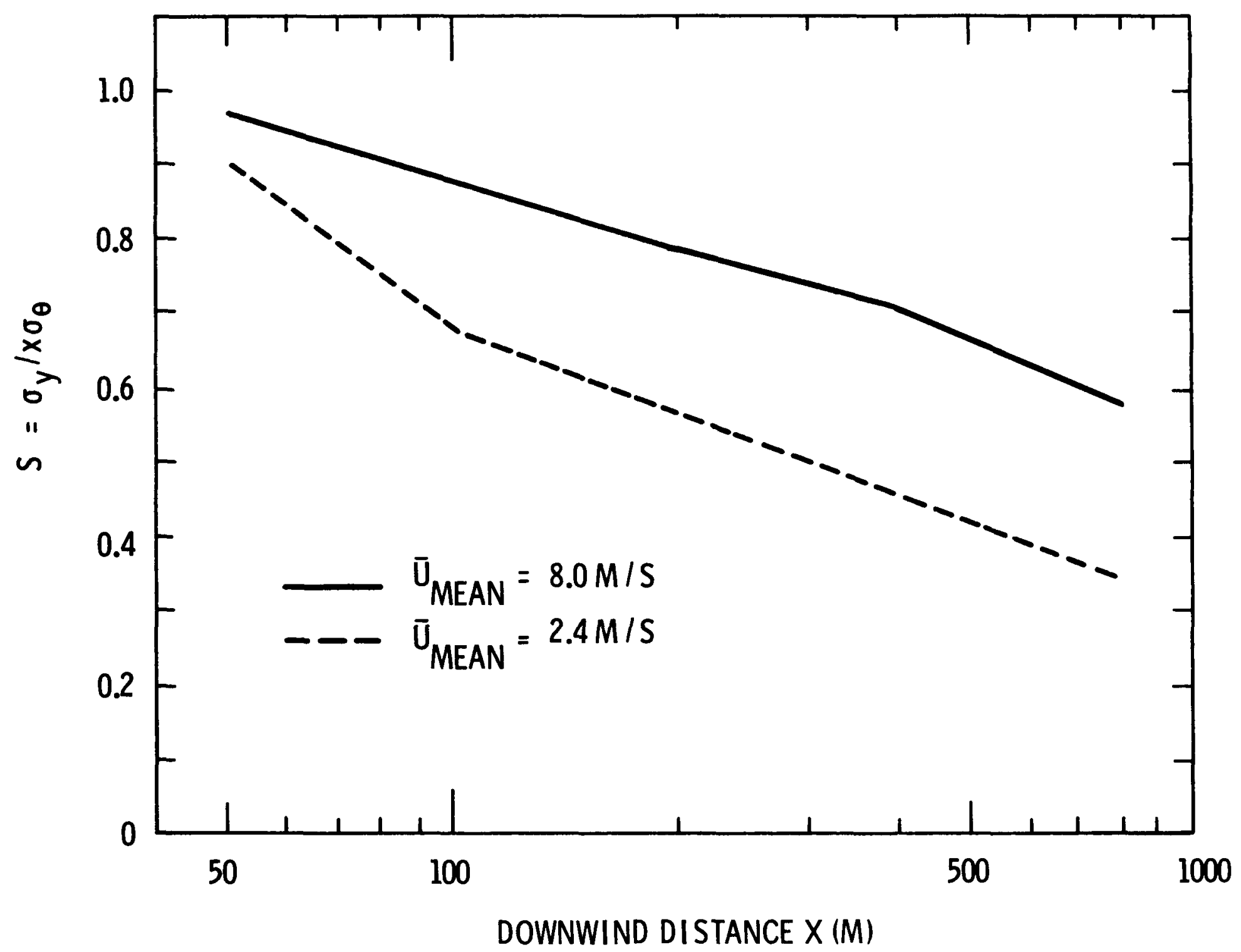

Figure 4.3. Variation of $S=\sigma / x \sigma$ with distance for two wind speed classes. Data are taken from Płoject Prairie Grass. 
were made: a) the stable spectrum can be described by the general expression given by Kaimal et al (1972) for frequencies above $f \sim .005$; b) the spectrum for the unstable case can be approximated by an expression similar to that used for the stable case, except that the frequency at which $\mathrm{nF}_{E}(n)$ attains its maximum value, $f_{\max }$, is shifted downward; c) a spectral gap exists at $f_{\text {min }}$, below which $n F_{E}(n)$ becomes larger as $f$ decreases; d) $n F_{E}(n)$ is a function of the dimensionless frequency $f$, even for the unstable case; and e) the Lagrangian and Eulerian spectra are related in the manner suggested by Hay and Pasquill (1959) over their whole frequency range, viz., $F_{L}(n)=\beta F_{E}(\beta n)$ with $\beta=4$.

The rate at which $\mathrm{nF}_{E}(n)$ decreases with frequency as the spectral minimum is approached from below is not well known. Kaimal et al (1976) suggest a -2 power dependence, while Hess and Clarke (1973) found values closer to -1.5. Panofsky and Van der Hoven (1955) and Smedman-Högström and Högström (1975) show a variety of spectra with various slopes. In several cases, a -1 power law seems to fit the data reasonably well. The actual slope chosen depends upon the frequency range below $f_{\min }$ one wishes to represent, since the curve becomes flatter as the minimum is approached. A value of -1 was adopted for our sample spectra.

As a working hypothesis, we therefore postulate the following forms as plausible approximations. For the stable case,

$$
n F_{E}(n)=\frac{6.25 \times 10^{-6}}{f}+\frac{f}{1+15.2 f^{5 / 3}}
$$

and for the unstable case,

$$
n F_{E}(n)=\frac{3.6 \times 10^{-9}}{f}+\frac{f}{1+10^{4} f^{5 / 3}}
$$

Eq. (4.9) applies to a spectrum with values of $f_{\max }=.25$ and $f_{\min }=.0025$ while (4.10) corresponds to $f_{\max }=.005$ and $f_{\min }=.00006$ (Kaimal et al, 1972; Smedman - Högström and Högström, 1975). These sample spectra are shown in Fig. 4.4. They are not normalized either on an absolute scale 


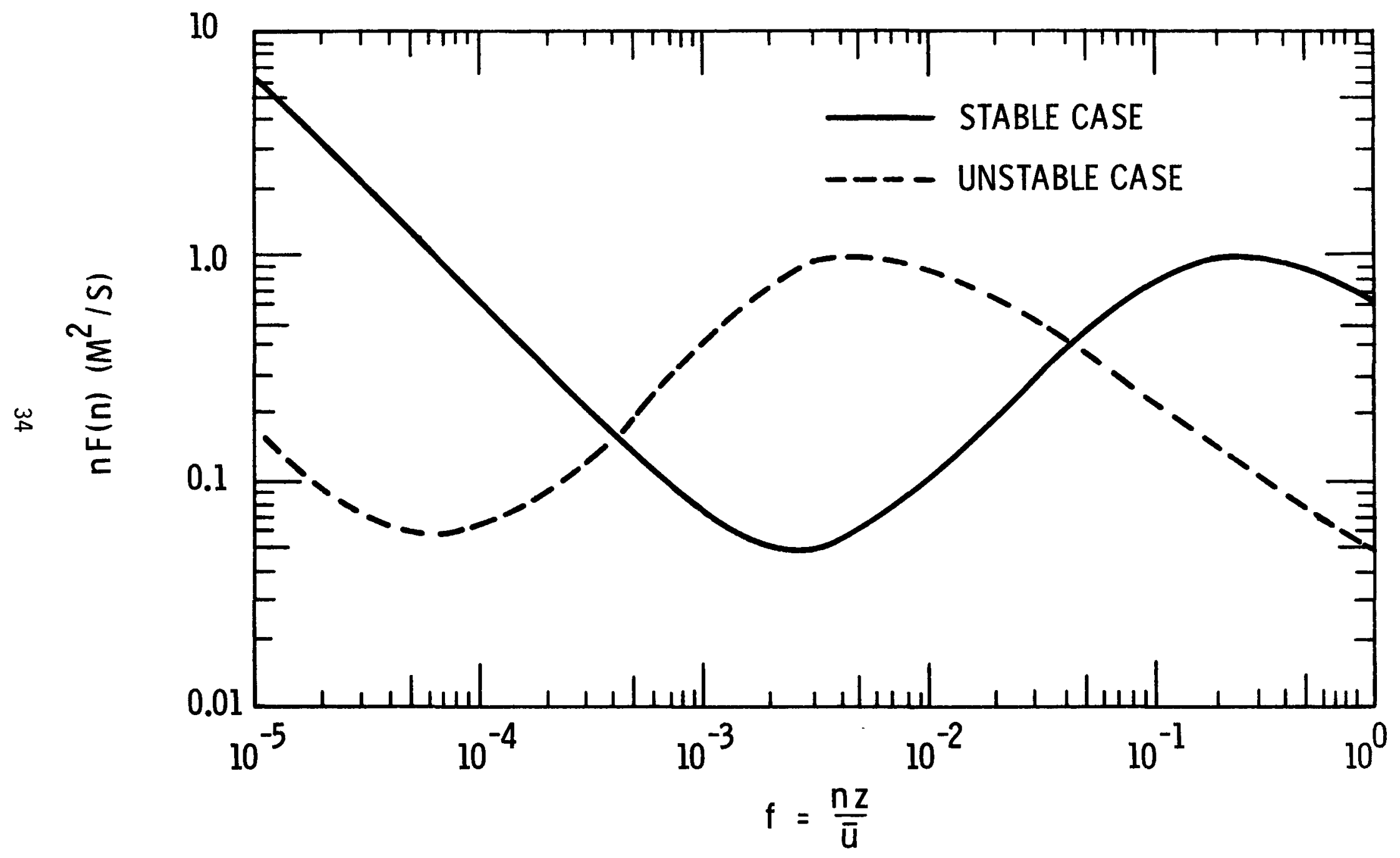

Figure 4.4. Sample spectra for stable and unstable conditions. Spectra not normalized. 
or with respect to each other, but this is immaterial since any normalizing factors would cancel upon application of (4.7) or (4.8).

Fig. 4.5 shows the behavior of $S$ obtained from these spectra, assuming an averaging time $t=5$ seconds and a mean velocity $u=5 \mathrm{~m} / \mathrm{s}$. Two curves are shown in each case, for sampling times $\tau$ of 600 and 1800 seconds. The curves extend only to a distance $x$ such that $T=x / u \leq \tau$.

A number of features are immediately apparent. For both stable and unstable spectra, longer sampling times generaliy imply larger values of $S$. (An exception to this may be noted for the stable spectrum at small downwind distances.) The values of $S$ determined from the unstable spectrum are larger close to the release point, while the stable spectrum produces larger values farther downstream. The crossover point depends upon sampling time; as $\tau$ increases, this point moves toward shorter distances. The exact behavior is, of course, dependent upon the actual form of the spectra, but a possible indication of this crossover phenomenon may be seen in Fig. 4.6. For the Prairie Grass results, the higher values of $S$ are associated with those runs taken during daylight hours, while the lower values of $S$ are associated with the more stable night runs. For the Green Glow-30 series, which has longer sampling times, the relative ordering of the curves with stability is reversed. This would be expected if the longer sampling times resulted in a crossover point at very short downwind distances. While the actual measured values of $S$ are not reproduced by (4.9) and (4.10), their qualitative behavior may be interpreted in terms of the crossover effect.

It is clearly possible to adjust the spectra to produce virtually any desired result, but such an exercise would be pointless. Rather, we have presented two cases which are plausible representations of previously observed spectra to demonstrate the importance of several parameters which have hitherto been neglected in describing the characteristics of plume dispersal. 


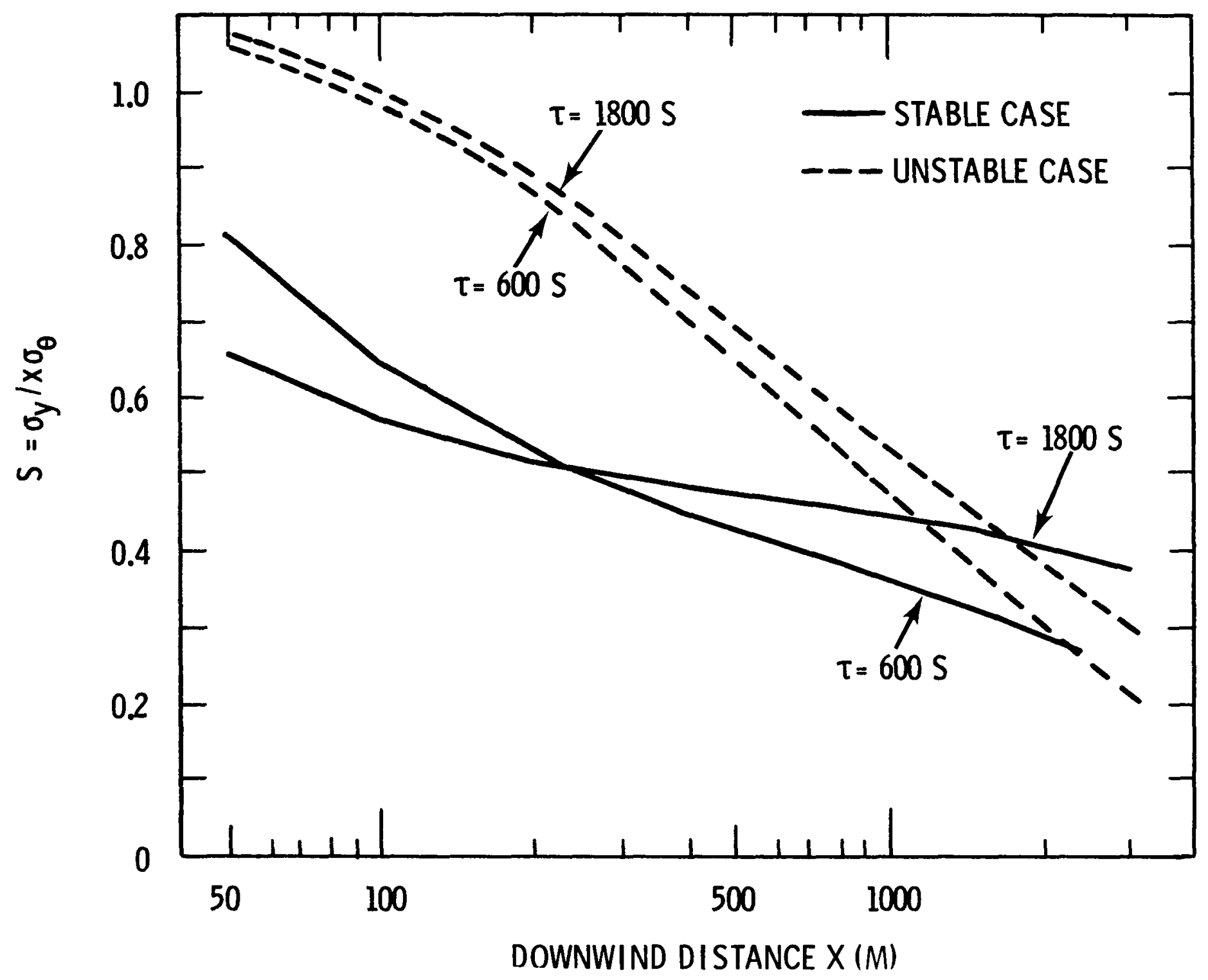

Figure 4.5. Variation of $S=\sigma / x \sigma$ with distance. Values are computed from sample spectra for two values of sampling time $\tau$. 

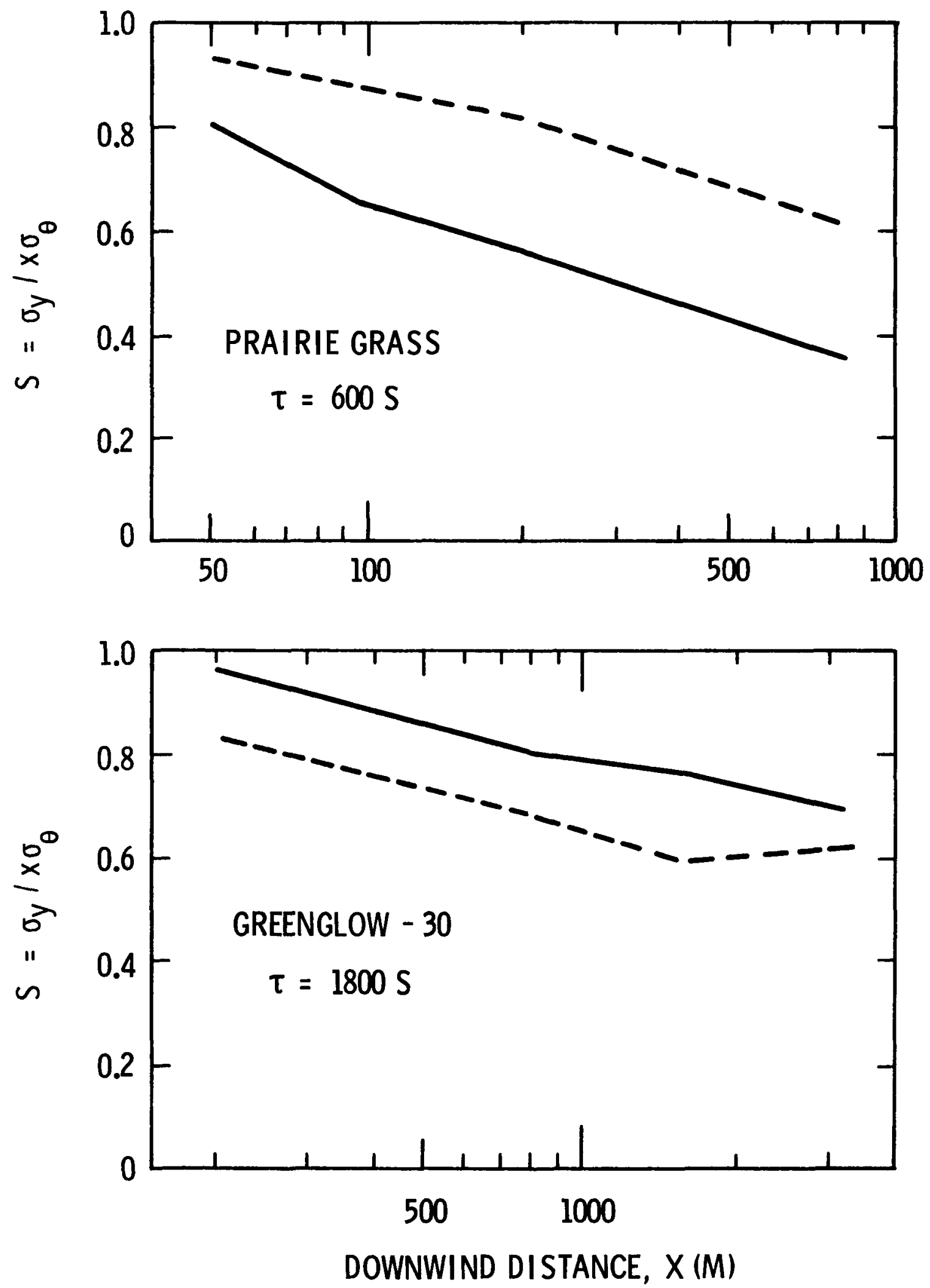

Figure 4.6. Variation of $S=\sigma_{y} / x \sigma_{\theta}$ with distance for stable (solid line) and unstable (dashed line) conditions. 


\subsection{EFFECTS OF RELEASE HEIGHT AND TERRAIN ROUGHNESS}

\subsubsection{Effects of Release Height}

The height from which a substance is released may also affect its subsequent dispersion as it is advected downwind. It has been suggested (Pasquil1, 1976) that, while $S$ is a function of $x$, this functional dependence is approximately independent of release height and surface roughness. If the dispersing plume is confined to a horizontal plane, e.g., near the surface, then $S$ can theoretically be evaluated through an application of Taylor's theorem. Within certain limitations, $\sigma_{\theta}$ is then shown to be a fair predictor of the dispersion. For elevated releases, however, the situation is considerably more complicated. As the plume descends toward the ground, it encounters turbulent fluctuations which vary with height (Kaimal et al, 1972). It is not at all evident, then, at what elevation $\sigma_{\theta}$ should be measured to provide useful predictions of diffusion at ground level.

Fig. 4.7 shows Hanford 67-Series results obtained for three release heights, where $\sigma_{y}$ and $\sigma_{\theta}$ are measured near the ground. As can be seen, the curves for the three release heights coincide only at some distance downstream from the source, and the distance to this point increases with increasing height of the release. Near the origin, the variation of $\sigma_{\theta}$ with height has a clear effect upon the behavior of S. Farther downstream, the lateral dispersion is dominated by the crosswind fluctuations near the ground, and the value of $\sigma_{\theta}$ at the release height is not a governing factor.

This interpretation is borne out by the results shown in Fig. 4.8, where $S$ is again plotted as a function of $x$, but $\sigma_{\theta}$ has been measured at the release height rather than near the ground. No apparent order can be seen in these curves, indicating that the value of $\sigma_{\theta}$ at elevated release points is not a good predictor of the ground-level dispersion. Moreover, the scatter of the data about the mean values is greater than for the analys is described by Fig. 4.7, particularly for the $26 \mathrm{~m}$ releases. Values of $\mathrm{S}$ calculated on the basis of $\sigma_{\theta}$ measured at both ground-level and release heights are summarized in Table 4.2 . 


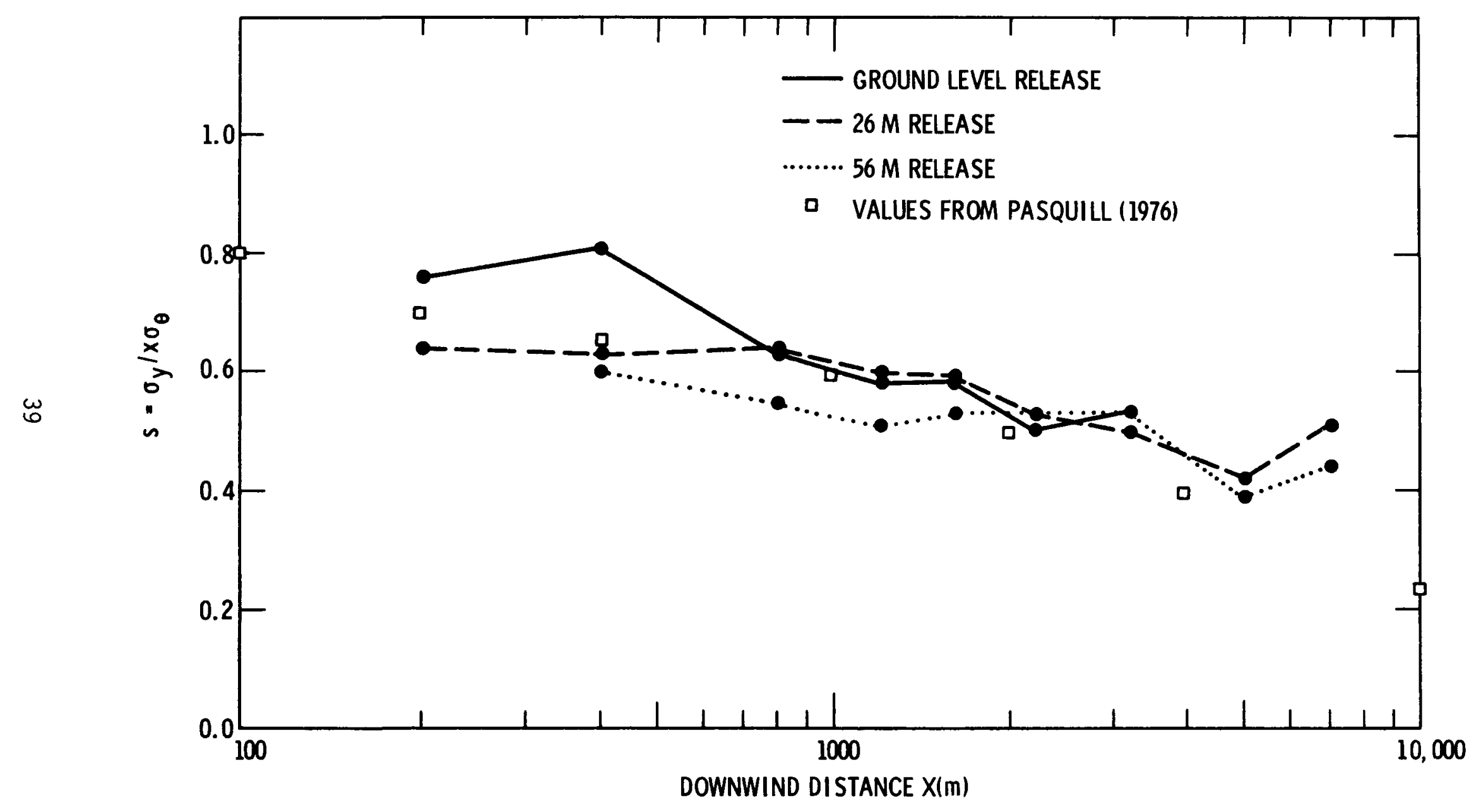

Figure 4.7. Variation of $S=\sigma_{y} / x \sigma_{\theta}$ with distance for three release heights, Hanford 67-Series. $\sigma_{\theta}$ was measured at $1.5 \mathrm{~m}$ elevation. 


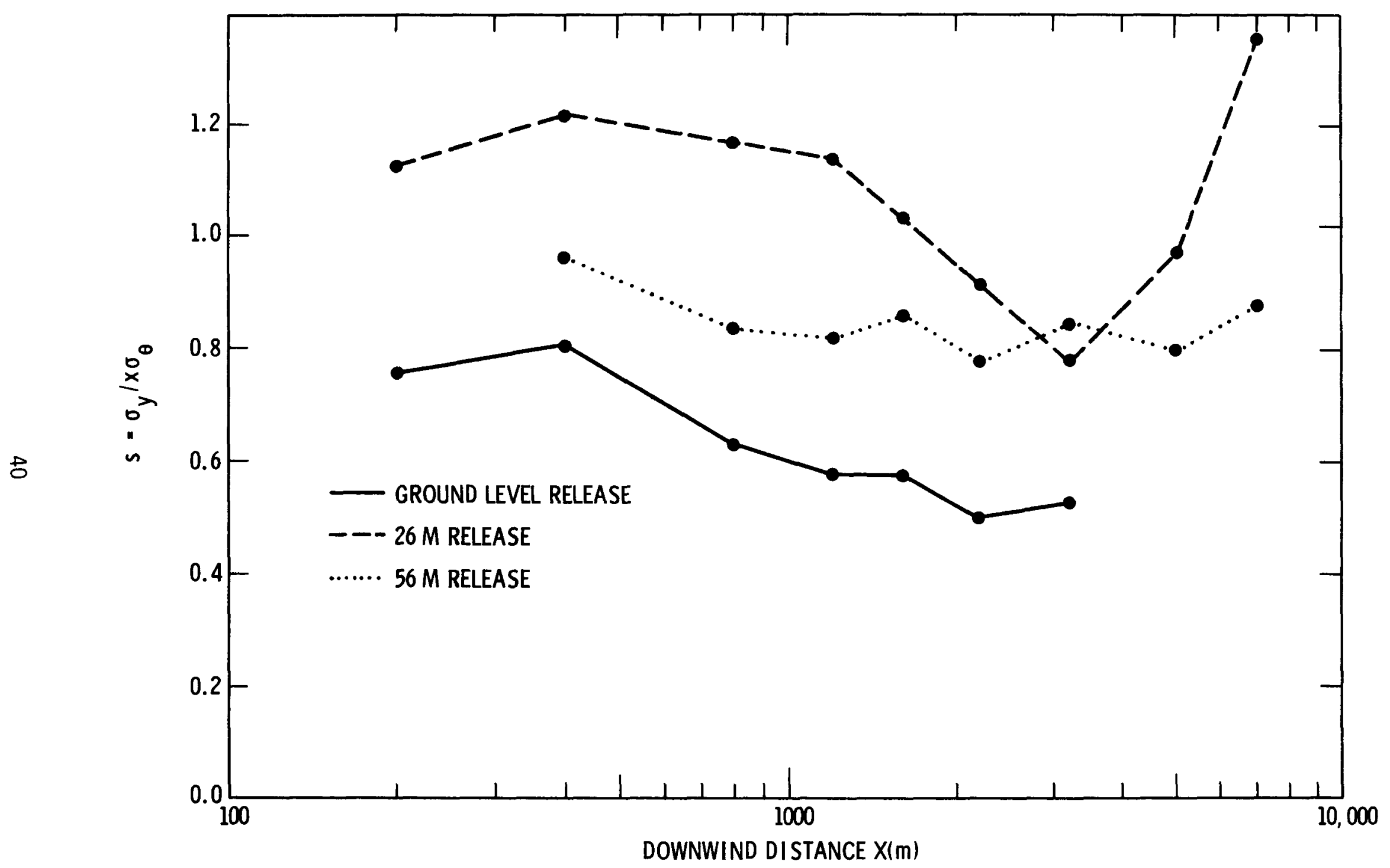

Figure 4.8. Variation of $S=\sigma_{y} / x \sigma_{\theta}$ with distance for three release heights, Hanford 67-Series. $\sigma_{\theta}$ was measured ${ }^{\theta}$ at release heights. 
Table 4.2 Values of S From Hanford 67-Series

Release

Height

Downwind Distance (m)

\begin{tabular}{lllllllll}
\hline 200 & 400 & 300 & 1200 & 1600 & 2200 & 3200 & 5000 & 7000 \\
\hline
\end{tabular}

$2 \mathrm{~m} \quad$ No. tests

$\begin{array}{rrr}5 & 4 & 15 \\ .76 & 81 & .63\end{array}$

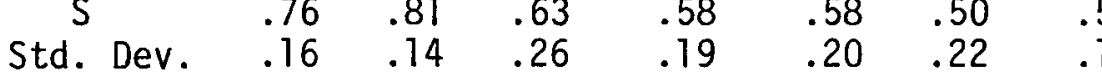

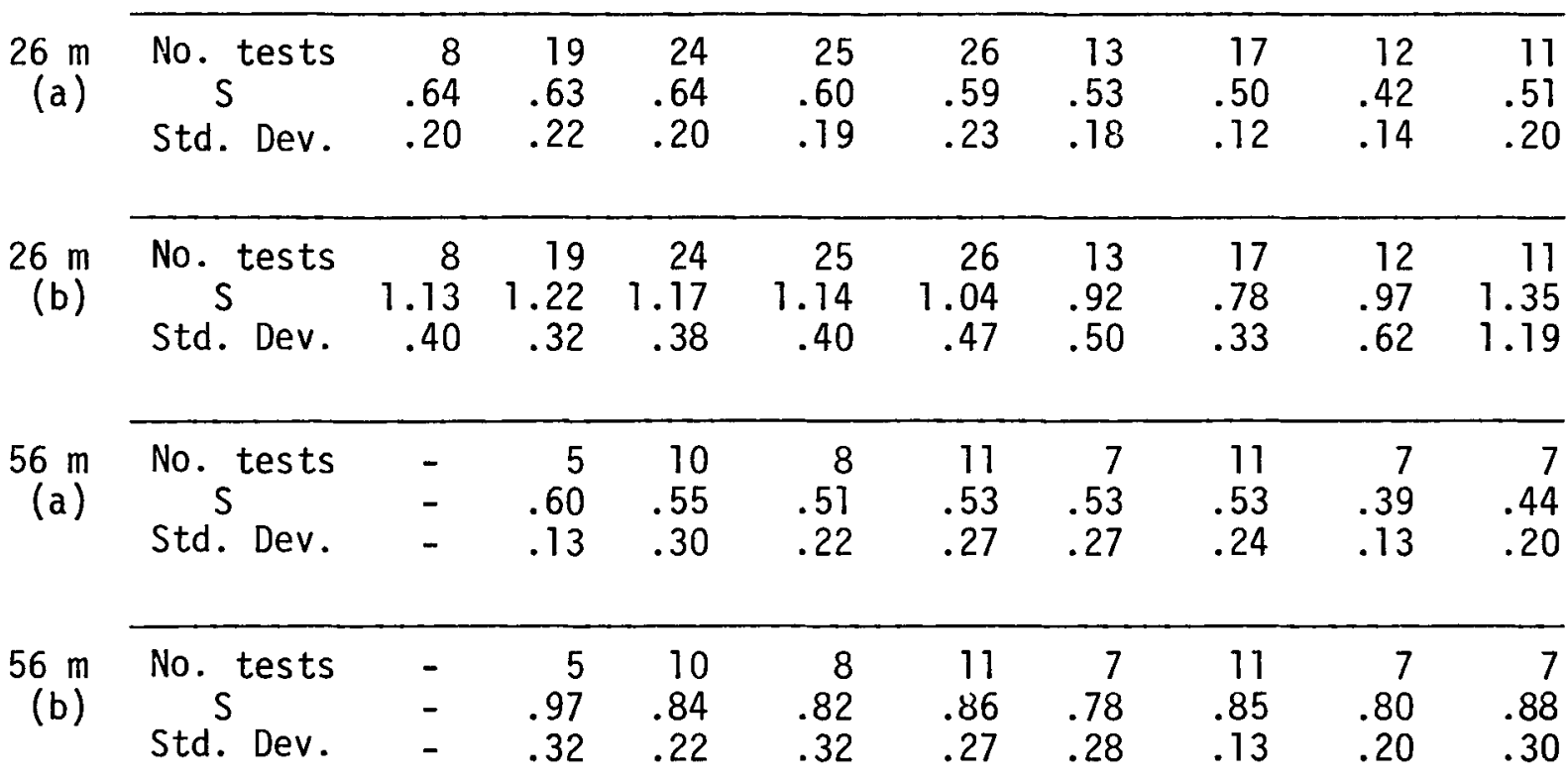

(a) $\sigma_{\theta}$ measured at $1.5 \mathrm{~m}$

(b) $\sigma_{\theta}$ measured near release height

\subsubsection{Effects of Terrain Roughness}

It has also been suggested (Pasqui11, 1974) that differences in terrain roughness would be reflected in differences in $\sigma_{y}$ and $\sigma_{\theta}$, but that the variation of $S$ with $x$ would be largely independent of such surface irregularities. Fig. 4.9 shows a comparison of results obtained for groundlevel releases at Hanford with those obtained from two other field programs carried out over considerably rougher surfaces. The former data are from the Hanford Green Glow-30 Series and Hanford 67-Series, while the latter are 


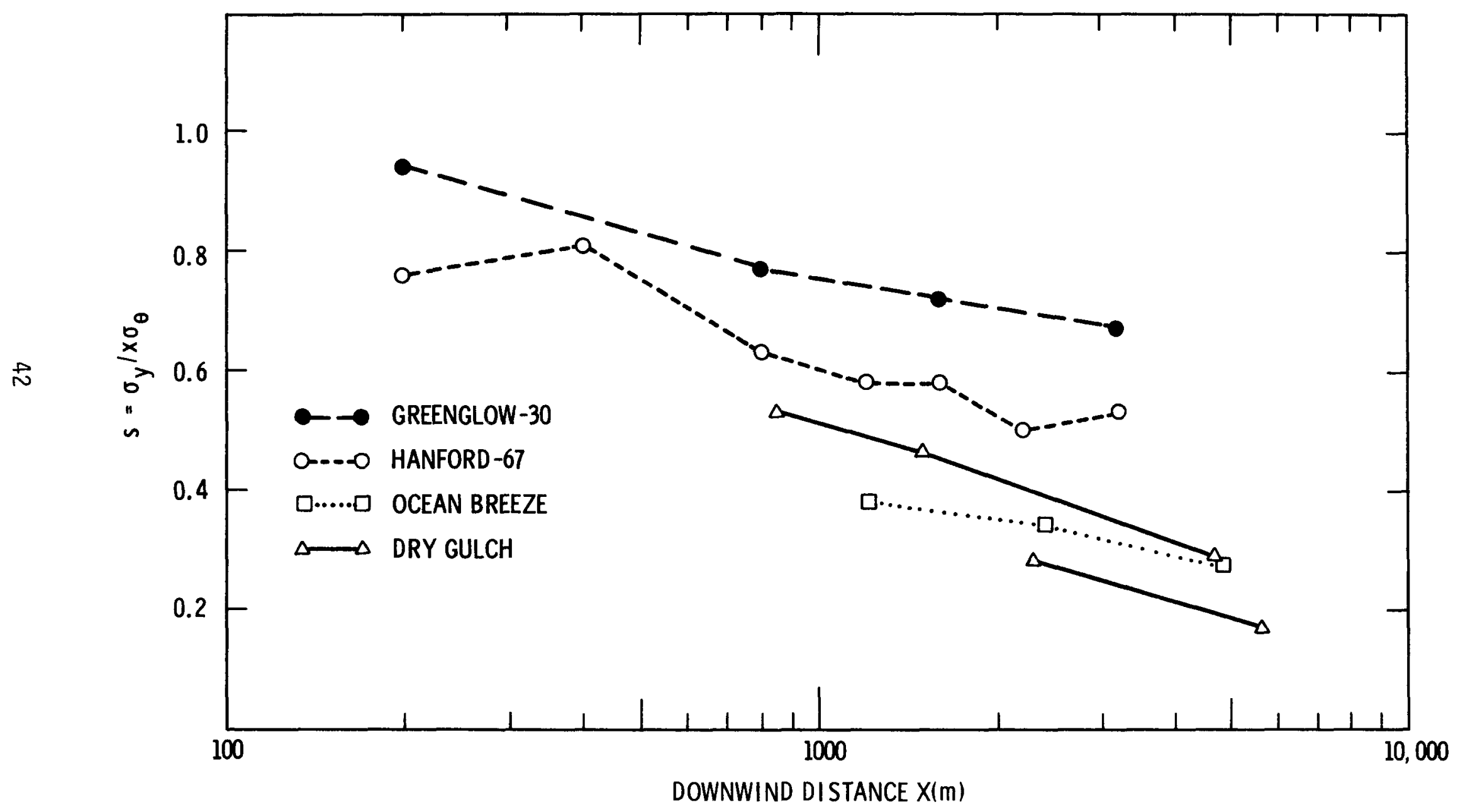

Figure 4.9. Variation of $S=\sigma_{y} / x \sigma_{\theta}$ with distance for field programs over terrain with different roughnesses. 
taken from the Dry Gulch and Ocean Breeze programs. The Ocean Breeze measurements took place in a region which was described as one of rolling terrain and dense vegetation, while the Dry Gulch data were collected in an area of complex terrain marked by rugged hills, ridges and ravines. The Hanford values lie consistently above the others.

The terrain descriptions given in the Dry Gulch and Ocean Breeze reports are misleading, however. While the general regions were not as flat as the Hanford diffusion grid, the particular areas in which the Dry Gulch measurements were taken were, in fact, relatively smooth. In particular, the terrain in the immedicate vicinity of the release points for both the Dry Gulch and Ocean Breeze programs was quite flat and unobstructed. Thus, interpretation of the reduced values of $S$ in terms of enhanced values of $\sigma_{\theta}$ because of terrain irregularities may not be justified. (Small scale terrain features might have affected $\sigma_{\theta}$ values because of contributions from small scale eddies. These eddies, however, would have been relatively ineffective in diffusing the plume, particularly at larger downwind distances.)

As suggested in Section 3.5, there is considerable suspicion about the accuracy of the wind fluctuation measurements in the Ocean Breeze and Dry Gulch programs. As a further check, some tests from the Mountain Iron series were analyzed to see if the rough terrain might be responsible for the observed reductions in $S$. The wind direction instrumentation for these tests was similar to that used in the Hanford studies.

Since the terrain made it impossible to make measurements along regular arcs, as in other programs, $\sigma_{y}$ values were generally determined from estimates of concentration isopleths at varying downwind distances. The results of 14 tests are shown in Fig. 4.10, together with a line representing best estimates of the behavior of $S$ taken from the analysis of the ground-level releases discussed previously. (Specific values of $S$ as a function of $x$ are given in Section 4.4.)

It is clear that there is no systematic tendency for $S$ to exhibit lower values than the "best" curve. This lends additional support to the suspicion that the Dry Gulch and Ocean Breeze results are anomalously low 


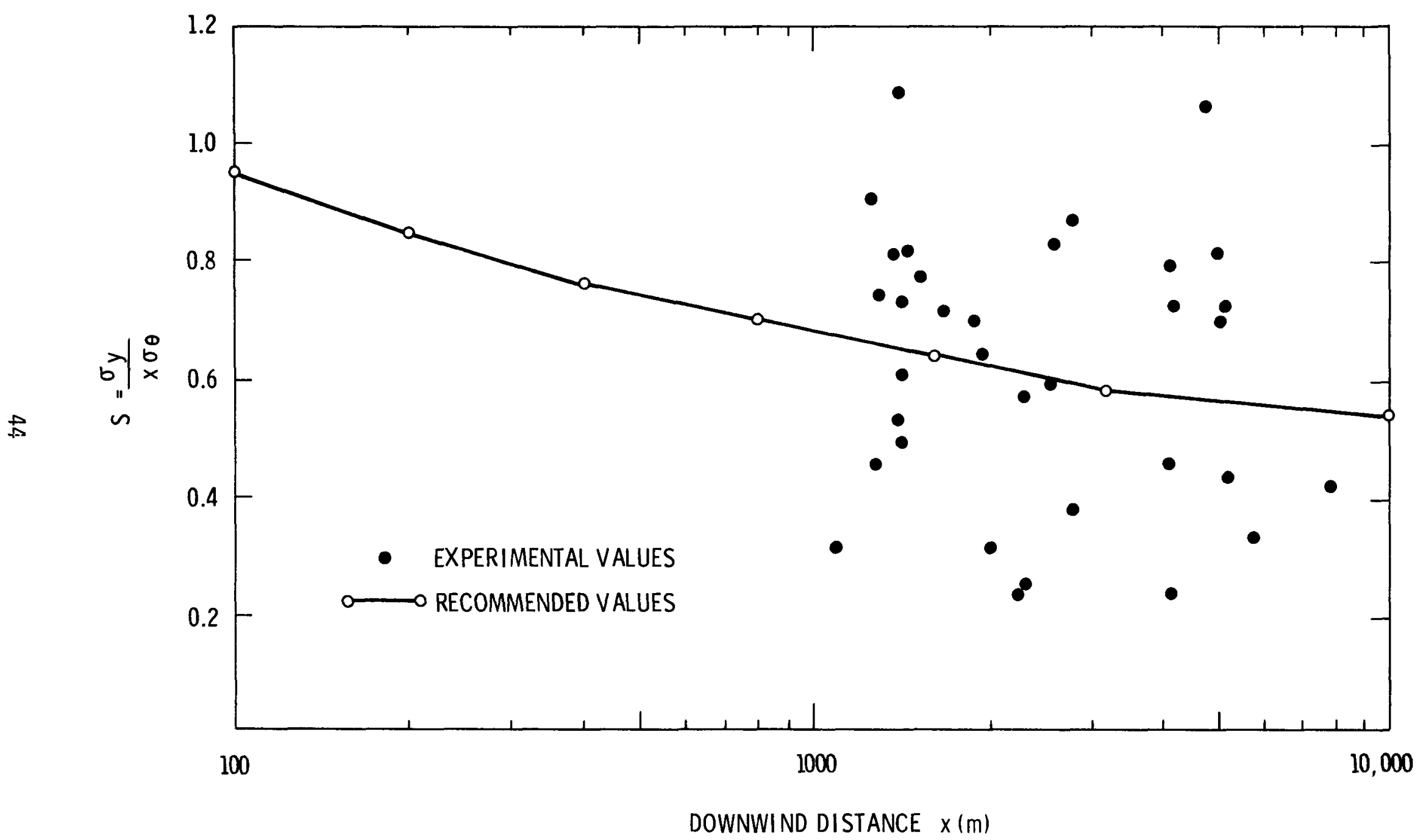
Figure 4.10. Comparison of curve for $S=\sigma / \sigma_{\theta}$ recommended for flat
homogeneous terrain with Mountain Iron datal 
due to an improperly functioning instrument. It also suggests that $\sigma_{\theta}$ continues to be a fair predictor of a plume's behavior, provided that the terrain remains relatively "homogeneous."

\subsubsection{Elevated Releases in Rough Terrain}

The Jülich and Karlsruhe programs involved tracer releases at heights of 50 and $100 \mathrm{~m}$ in regions of quite rough terrain. The Jülich investigators found evidence for increased dispersion which they attributed to roughness effects; additional details are presented in Section 5.4 on vertical dispersion characteristics for elevated sources.

For the Jülich tests, values of $\sigma_{\theta}$ are given at the release height, but not at the ground. Comparisons of $S$ from Hanford 67, NRTS and Juitich data are shown in Fig. 4.11 with $\sigma_{\theta}$ measured at release height. The Jülich data fall well below the other results, but it is difficult to provide a clear interpretation of this. It was suggested earlier that values of $\sigma_{\theta}$ measured at elevated release points (as is the case for the results in Fig. 4.11) do not seem to be very useful as predictors of plume spread. However, in the case of very rough terrain, a measurement of $\sigma_{\theta}$ near the ground may also not be representative of the turbulent eddies which the plume experiences as it travels downwind. In addition, it is curious that the fit to the NRTS data, indicated by the dashed line, is so close to the Hanford results, since $\sigma_{\theta}$ at the release height does not seem to be a reliable predictor of the Hanford data.

\subsection{CONCLUSIONS}

The relationship between $S=\sigma_{y} / x \sigma_{\theta}$ and $x$ as proposed by Pasquill is approximately correct for a wide range of conditions, but a more precise formulation of this dependence must take into account a number of factors previously disregarded. Sampling time, averaging time, wind speed, stability, release height, and perhaps terrain roughness may all have important effects. In particular, a knowledge of $\sigma_{\theta}$ is not sufficient to specify $\sigma_{y}$, although in the absence of more detailed information, it is 


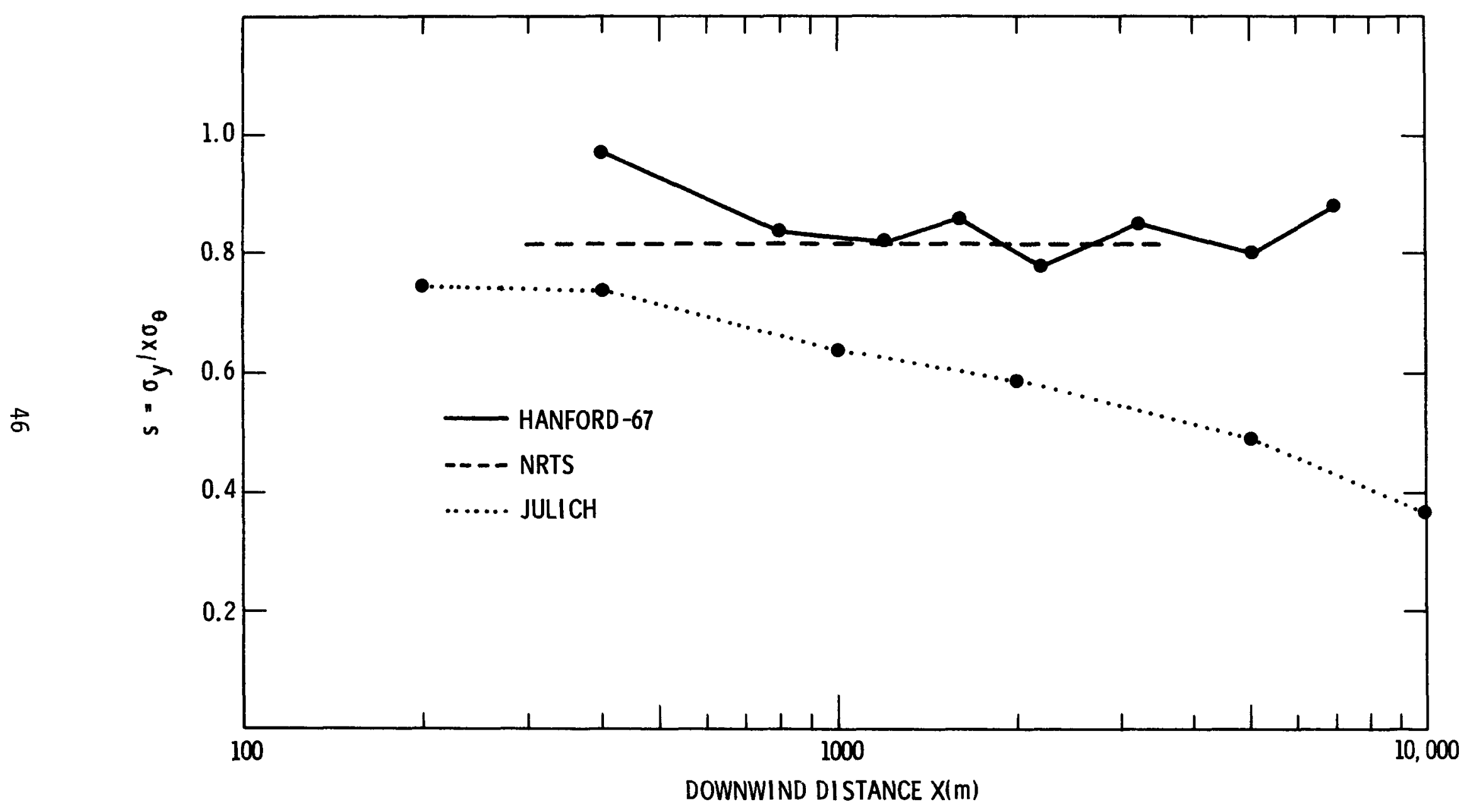

Figure 4.11. Variation of $S=\sigma_{y} / x \sigma_{\theta}$ with distance for three field programs with release heights of $\sim 50 \mathrm{~m}$, but different roughnesses. $\sigma_{\theta}$ was measured at release height. 
useful for a rough estimation of $\sigma_{y}$ for ground-level releases.

Until additional studies are performed, we recommend some modifications in the values of $S$ originally suggested by Pasquill. For an averaging time of 5 seconds for $\sigma_{\theta}$, the suggested variation of $S$ with $x$ is given in Table 4.3 for two sampling times, 1800 and 3600 seconds. These values are applicable only for flat homogeneous terrain and for releases near the ground.

Table 4.3. Recommended Values of $S=\sigma / 1 \times \sigma o$ for $t=5$ seconds.

\begin{tabular}{cccccccc}
\hline$x(\mathrm{~km})$ & .1 & .2 & .4 & .8 & 1.6 & 3.2 & $10^{\mathrm{a}}$ \\
\hline $\mathrm{S}(\tau=1800 \mathrm{~s})$ & .95 & .85 & .76 & .70 & .64 & .58 & .52 \\
$\mathrm{~S}(\tau=3600 \mathrm{~s})$ & 1.04 & .98 & .92 & .85 & .77 & .67 & .54 \\
\hline
\end{tabular}

\section{(a) Extrapolated}

For ground-level releases over moderately rough but homogeneous terrain, the values of Table 4.3 probably provide reasonable estimates as well.

For elevated releases in smooth terrain, $\sigma_{\theta}$ at the release height does not appear to be a reliable parameter for use in diffusion estimates. The surface value of $\sigma_{\theta}$ is not appropriate at small distances from the source but becomes more useful farther downwind.

For elevated releases in rough terrain, the only data available are from the Julich tests. They differ significantly from the results found over smooth terrain, but are not readily interpreted in terms of $\sigma_{\theta}$. An alternate approach is described in Section 5.4 .

A note of caution should be interjected concerning the release height study based on the Hanford 67-Series. The great majority of tests were carried out in neutral to moderately stable conditions; unstable stratifications were rarely encountered. It is possible that some of the effects found, particularly those noted in Fig. 4.8, may be influenced to some extent by this bias toward more stable cases. 


\section{VERTICAL DISPERSION}

\subsection{INTRODUCTION}

In this chapter the vertical dispersion will be studied independently of the latera7. One method is the direct determination of $\sigma_{z}$ from the vertical distribution of tracer, as done for $\sigma_{y}$ in the previous chapter. In the absence of remote sensing techniques, this approach requires a dense array of towers or other suitable detector configurations. For ground-level releases under lapse conditions, and for elevated releases under all conditions, the towers must be of considerable height if a significant fraction of the diffusing plume is to be embraced by the sampling network. Hence data of this type is expensive to obtain and quite scarce.

A second method is to utilize the crosswind-integrated, ground-level concentrations (CWIC) to infer the vertical dispersion. This approach requires the assumption of tracer conservation (or an accounting for tracer losses), assumption of a form for the vertical tracer distribution, and estimation of the vertical profile of the wind. In contrast to the direct analysis of the lateral and vertical distributions, study of the CWIC also requires reliable absolute values of tracer concentration. However, the ground-level concentration, not the vertical spread, is usually the quantity of practical interest. The CWIC is also less susceptible to errors introduced by statistical fluctuations, instrument malfunctions, or analytical uncertainties associated with the use of data from only one or a few crosswind locations.

In the following section, equations are developed from Lagrangian similarity theory for the prediction of the CWIC for ground-level sources. This enables much more extensive verification of the theory than would be possible by comparing measured and predicted values of $\sigma_{z}$. The more limited vertical distribution data are used to supplement the CWIC data where needed.

A similar mix of data is found in Section 5.3 for elevated releases, where vertical tracer distributions are used to verify the more plentiful $\sigma_{z}{ }^{\prime} s$ inferred from the CWIC. No organizing theory is proposed in that 
section, but the observations from the Hanford 67-Series for different release heights are compared to each other and to those predicted for ground-level releases. In Section 5.4, elevated release data from other field series, particularly the jülich data for rougher terrain, are discussed. However, the format of the published results in general precludes an in-depth comparison amonq different sites.

\subsection{GROUND-LEVEL SOURCES}

\subsubsection{Background}

Monin-Obukhov similarity theory proposes that the properties of atmospheric turbulence within the surface flux layer are determined solely by the height $z$, the surface shearing stress $\tau$, the vertical heat flux $H$, and the quantities $\rho, c_{p}$ and $g / \theta$. Equivalently, these may be reduced to $z$, the friction velocity $u_{*}$, and the Obukhov length L. Lagrangian similarity theory extends this proposal to the diffusion of passive contaminants with in the surface flux layer. Chaudhry and Meroney (1973) have shown that Lagrangian similarity theory can predict the vertical spread of a material diffusing into the atmosphere from a ground-level source, if it is assumed that the eddy diffusivity of the material corresponds to that of heat rather than that of momentum. They compared the predicted mean height of the diffusing material $\bar{z}$ with that measured $100 \mathrm{~m}$ downwind of the source during the Prairie Grass diffusion experiments.

The Prairie Grass experiments also measured the horizontal distribution of the diffusing tracer at a height of $1.5 \mathrm{~m}$ and at 5 distances ranging from $50 \mathrm{~m}$ to $800 \mathrm{~m}$ from the source. Hence a comparison of the measured and predicted values of the CWIC can test Chaudhry and Meroney's eddy diffusivity proposal at larger distances from the source. Further, since the surface flux layer has a finite depth ranging from a few meters under stable conditions to perhaps $200 \mathrm{~m}$ under convective conditions, the diffusion will eventually extend above the surface layer. This comparison can therefore also investigate the downwind range of applicability of the surface similarity description of vertical diffusion. 
The vertical spread of the contaminant and the CWIC are predicted by Lagrangian similarity theory to also be a weak function of the surface roughness $z_{0}$. This functional dependence on roughness can be tested with the surface-source diffusion measurements made at NRTS, Idaho Falls, Idaho and with the Green Glow-30 Series data from Hanford, Washington. At these sites $z_{0}$ is $1.5 \mathrm{~cm}$ and $3 \mathrm{~cm}$, respectively, factors of 2.5 and 5 greater than the $0.6 \mathrm{~cm}$ value for the Prairie Grass site.

\subsubsection{Prediction of the ground-1evel concentration}

Lagrangian similarity theory (Pasqui11, 1974) assumes that the mean rate of vertical displacement for particles released from ground level is

$$
\frac{d \bar{z}}{d t}=a u_{\star} \Phi(\bar{z} / L)
$$

where $\Phi$ is a function of atmospheric stability equal to unity for adiabatic conditions. The mean rate of horizontal displacement is assumed equal to

$$
\frac{d \bar{x}}{d t}=u(c \bar{z})
$$

Given the constants $a$ and $c$ and functional forms for $\Phi$ and the wind profile, $\bar{z}$ may then be determined as a function of $\bar{x}, u_{*}$ and $L$.

Assuming that vertical diffusion from a ground-level source can also be described by K-theory, Chaudhry and Meroney (1973) have shown that

$$
\frac{d \bar{z}}{d t} \simeq k u_{\star} / \phi(\bar{z} / L)
$$

where $\phi$ is also unity for adiabatic conditions and describes the dependence on atmospheric stability of the vertical eddy diffusivity of the contaminant, 


$$
K=u_{\star} k z / \phi(z / L)
$$

Combination of (5.2) and (5.3) leads to

$$
\bar{x}=\frac{1}{u_{\star} k} \int_{z_{0}}^{\bar{z}} u(c z)_{\phi}(z / L) d z \text {. }
$$

Chaudhry and Meroney use $\phi_{h}$ in (5.4) and (5.5), following the suggestion of Monin and Yaglom (1965) that the eddy diffusivities of heat and a passive substance are equal. This choice is supported by Crawford (1965) and Dyer and Hicks (1970) who found that the eddy transfer mechanisms of heat and water vapor were the same, by Galbally (1971) who came to a similar conclusion for the transfer of heat and ozone, by Sinclair et al (1975) for heat, water vapor and carbon dioxide, and by Chaudhry and Meroney's analysis of the Prairie Grass $\mathrm{SO}_{2}$ diffusion data.

By assuming a vertical distribution of the material equal to

$$
x(x, z)=x(x, z=0) F(z / \bar{z}, \bar{z} / L),
$$

the crosswind-integrated concentration $x(x, z)$ may also be predicted from Lagrangian similarity theory (Monin and Yag7om, 1965). However, MaIhotra and Cermak (1964), simulating atmospheric diffusion from a surface source in a wind tunnel for both neutral and unstable conditions, found that the effect of instability was to increase the scale of the diffusion without altering the form of the diffusing plume. Hence the influence of $\bar{z} / L$ on $F$ appears to be quite weak.

Malhotra and Cermak's data fit the form

$$
x(x, z)=x(x, z=0) \exp \left[-(z / b \bar{z})^{r}\right]
$$

with a value of $r=1.4$ appropriate to both neutral and unstable conditions. 
Pasquill (1974) reports that for neutral conditions a value of $r=1.15$ was found $100 \mathrm{~m}$ downwind of the source during 7 tracer releases at Porton, England, and a value of 1.5 was found at $229 \mathrm{~m}$ during 29 releases at Cardington, England. Elliot (1961) has investigated the vertical distribution of tracer measured during Prairie Grass at $100 \mathrm{~m}$ downwind of the source and reported an average value of $r=1.5$ for the 41 releases analyzed. He found that a Gaussian value of 2 for $r$ is an overestimate except in fairly stable atmospheric conditions, that 1.5 was more appropriate for near-neutral conditions, and that $r$ was less than 1.5 for unstable conditions.

The constant b of Eq. (5.7) is determined from the definition of $\bar{z}$,

$$
\bar{z}=\int_{0}^{\infty} z \chi(x, z) d z / \int_{0}^{\infty} x(x, z) d z \text {, }
$$

to be equal to $\Gamma(1 / r) / \Gamma(2 / r)$, where $\Gamma$ is the gamma function. $\chi(x, z=0)$ is determined by the continuity condition

$$
\int_{0}^{\infty} u(z) x(x, z) d z=Q
$$

Substituting (5.7) into (5.9) and putting the result in dimensionless form,

$$
\begin{array}{r}
{\left[\frac{u_{\star} z_{0}}{k} \frac{x(x, z=0)}{Q}\right]^{-1}=\int_{1}^{\infty}\left[f(z / L)-f\left(z_{0} / L\right)\right]} \\
x \exp \left[-(z / b \bar{z})^{r}\right] d\left(z / z_{0}\right)
\end{array}
$$

where $f$ is a dimensionless function describing the wind profile,

$$
u(z)=\frac{u_{*}}{k}\left[f(z / L)-f\left(z_{0} / L\right)\right] .
$$


Finally, the constant $c$ in $(5.2)$ is determined by calculating the mean advection velocity,

$$
u(c \bar{z})=\int_{0}^{\infty} u(z) x(x, z) d z / \int_{0}^{\infty} x(x, z) d z .
$$

Again using (5.7),

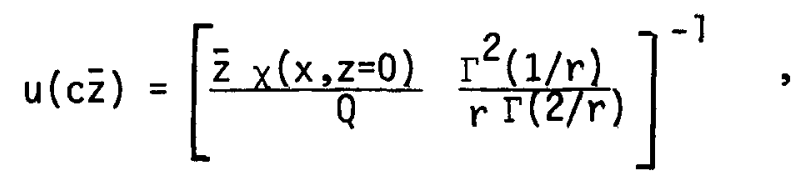

which may be evaluated with the aid of (5.10).

The quantity $u_{\star} z_{0} x(x, z=0) / k Q$ has been calculated as a function of $x / z_{0}$ and $z_{0} / L$ from $(5.5),(5.10)$ and (5.13), assuming a value of $r=1.5$ and equality of the diffusivities of matter and heat. Specifically, Businger et al's (1971) formulas for $\phi_{h}$ and $\phi_{m}$ have been used, along with Paulson's (1970) integral of the Businger et al formula for $\phi_{\mathrm{n}}$ to get $f$ :

$$
\left.\begin{array}{rl}
\phi_{h}(\zeta)= & 0.74+4.7 \zeta \\
f(\zeta)= & \ln +4.7 \zeta \\
\phi_{h}(\zeta)= & 0.74(1-9 \zeta)-1 / 2 \\
f(\zeta)= & \ln \zeta-2 \ln [(1+\psi) / 2]+ \\
& \ln \left[\left(1+\psi^{2}\right) / 2\right]-2 \tan ^{-1}
\end{array}\right\} \zeta \geq 0
$$

where $\zeta=z / L, \psi=(1-15 \zeta)^{1 / 4}$ and $k=0.35$. The results are presented in Fig. 5.1. Calculations have also been made assuming values of 1 and 2 for $r$, assuming that the eddy diffusivity of matter is equal to that of momentum, ana using Dyer's (1974) formulas for $\phi_{h}$ and $\phi_{m}$. Good comparisons with the Prairie Grass data were found for both $r=1.5$ and $r=2$ and for both 


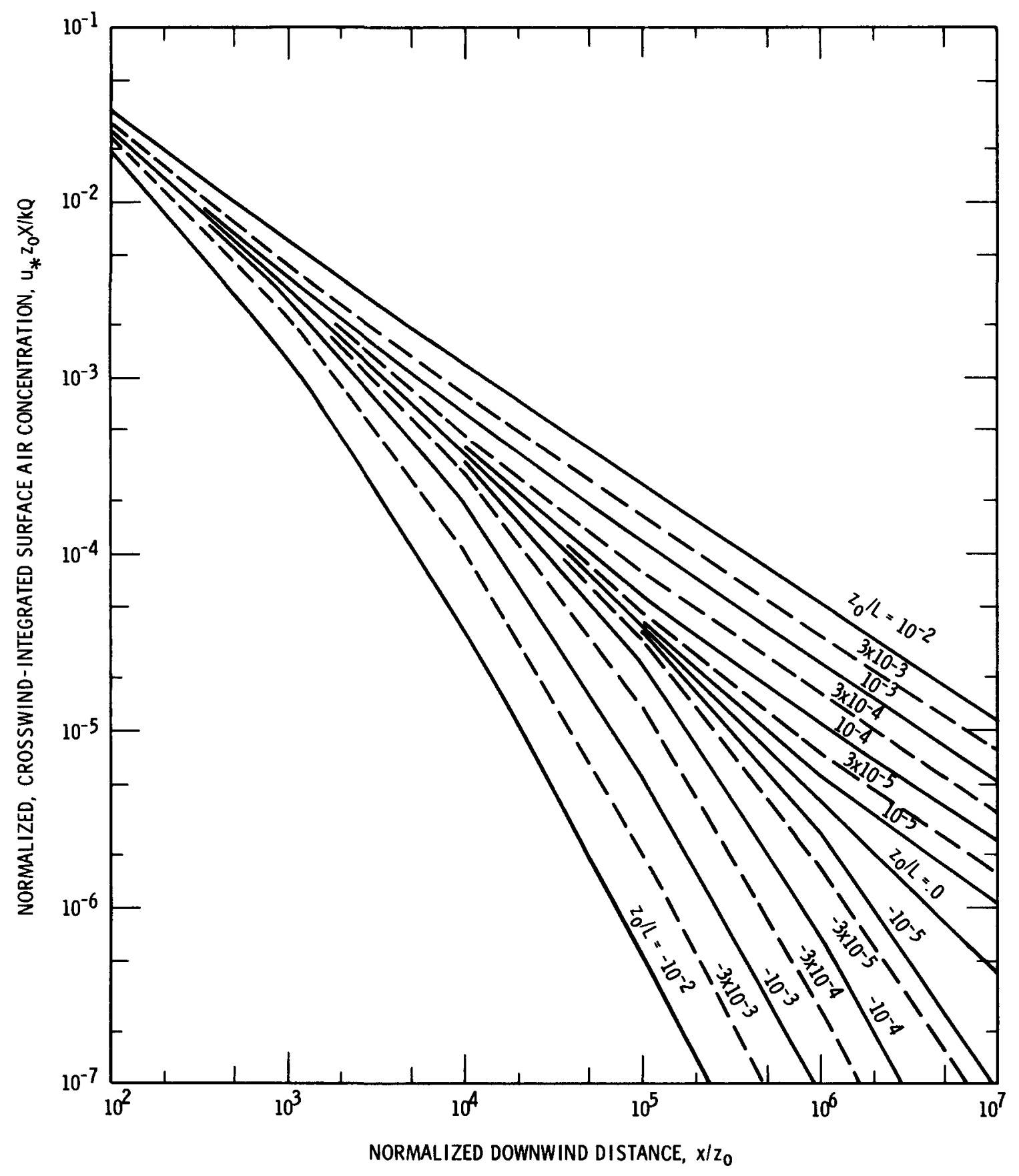

Figure 5.1. Predicted CWIC at ground-level as a function of downwind distance for various stability conditions. 
the Businger et al and Dyer formulas, assuming equal diffusivities for matter and heat. Noticeably poorer comparisons were found with either $r=1$ or the assumption of equal diffisivities for matter and momentum.

\subsubsection{Comparison with Prairie Grass Observations}

During Project Prairie Grass, tracer concentrations were measured at a height of $1.5 \mathrm{~m}$ along arcs at distances of $50 \mathrm{~m}, 100 \mathrm{~m}, 200 \mathrm{~m}, 400 \mathrm{~m}$, and $800 \mathrm{~m}$ from the source. These ground-level measurements were summed along each arc and divided by the source strength to give the normalized CWIC at each downwind distance. Concurrent vertical profiles of wind speed and temperature were used to estimate $L$ and $u_{\star}$ for each diffusion experiment. More details of the meteorological data analysis are presented in Chapter 3 and the derived data are tabulated in Appendix $A$.

In Figs. 5.2-5.6 the observed $u_{\star} x(x, z=1.5 \mathrm{~m}) / Q$ are plotted as a function of atmospheric stability parameterized by $1 / \mathrm{L}$. (For $z_{0}=0.6$ $\mathrm{cm}$, Golder (1972) finds Pasquill diffusion class $A$ to correspond to $1 / \mathrm{L}<-.14 \mathrm{~m}^{-1}$, class $C$ to $-.06 \mathrm{~m}^{-1}<1 / L<-.02 \mathrm{~m}^{-1}$, and class $E$ to $.02 \mathrm{~m}^{-1}<1 / \mathrm{L}<.08 \mathrm{~m}^{-1}$.) The open circles denote cases where the computed $L$ varied by more than a factor of 2 between the heights of $1 \mathrm{~m}$ and $4 \mathrm{~m}$, indicating that the empirical formulas for $\phi_{m}$ and $\phi_{h}$ were not compatible with the observed profiles of wind and temperature. This occurs mostly for strongly stable conditions when the surface flux layer may be quite shallow compared to the height of the profile measurements and the vertical extent of the diffusing plume.

The predictions of Lagrangian similarity theory, calculated as in Fig. 5.1 are shown as solid curves in Figs. 5.2-5.6. The comparison with the diffusion data is good. Figure 5.7 shows the predicted values of $x(x, z=1.5) / Q$ plotted against the measured values for all downwind distances; the 1 ine of equality is shown for reference. As also seen in Figs. 5.2-5.6, there is a tendency for the predictions to be high for small values of $x / Q$ and to be low for large values of $x / Q$. A 11 but 20 predictions out of $333,94 \%$, fall between a factor of $1 / 2$ and 2 times the measured value. In 


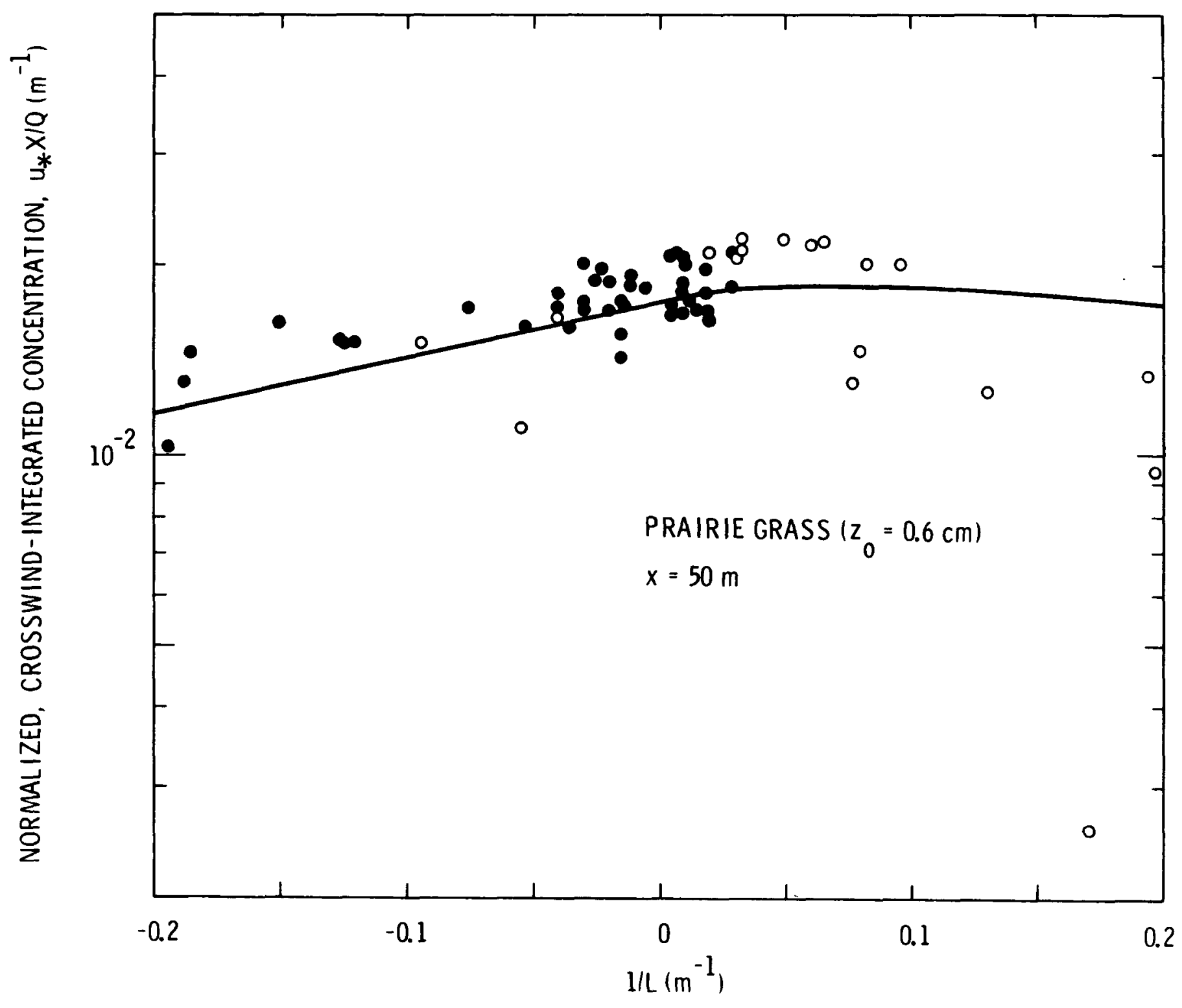

Figure 5.2. Predicted and measured CWIC $50 \mathrm{~m}$ downwind of the source as a function of atmospheric stability, Project Prairie Grass. 


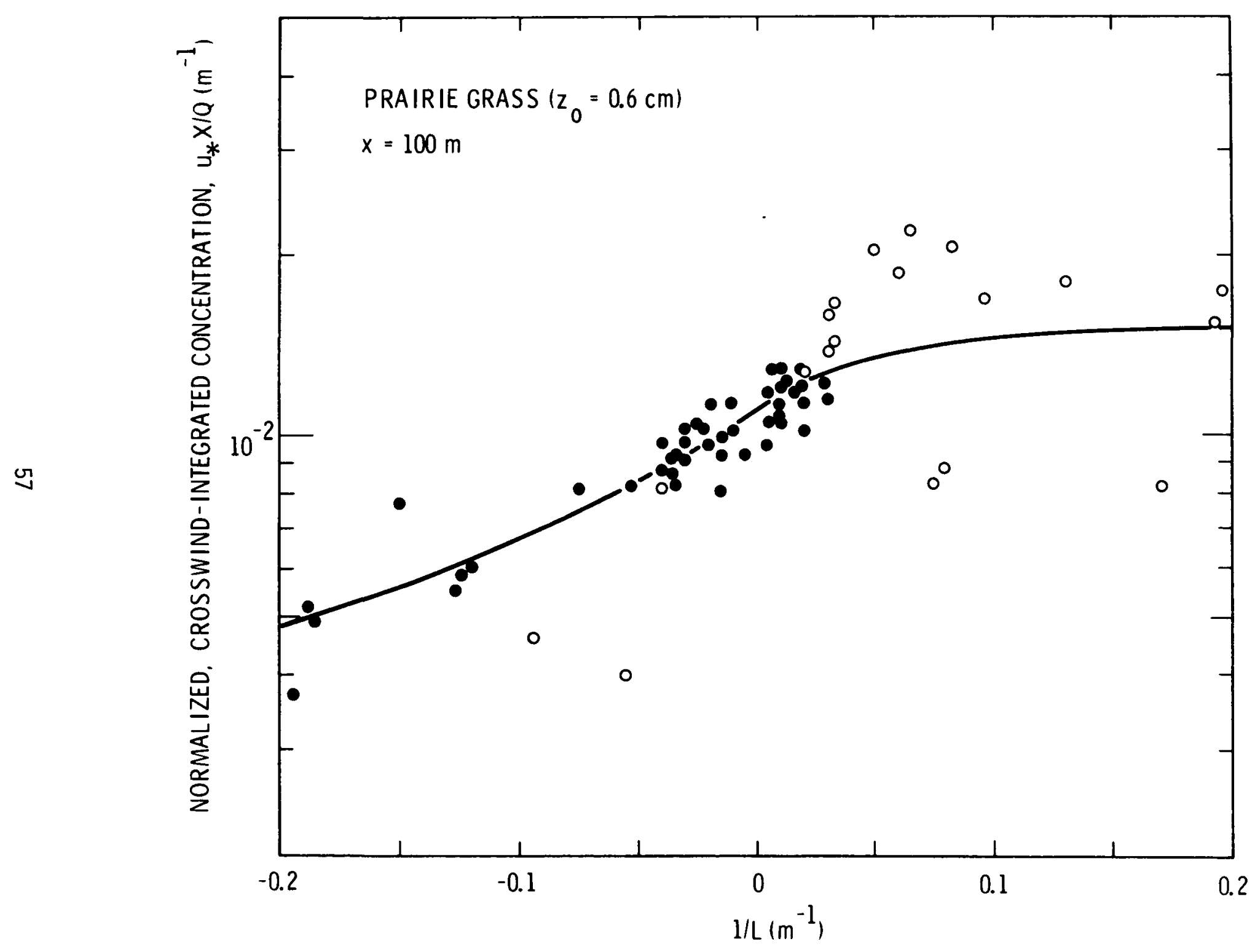

Figure 5.3. Predicted and measured CWIC $100 \mathrm{~m}$ downwind of the source as a function of atmospheric stability, Project Prairie Grass. 


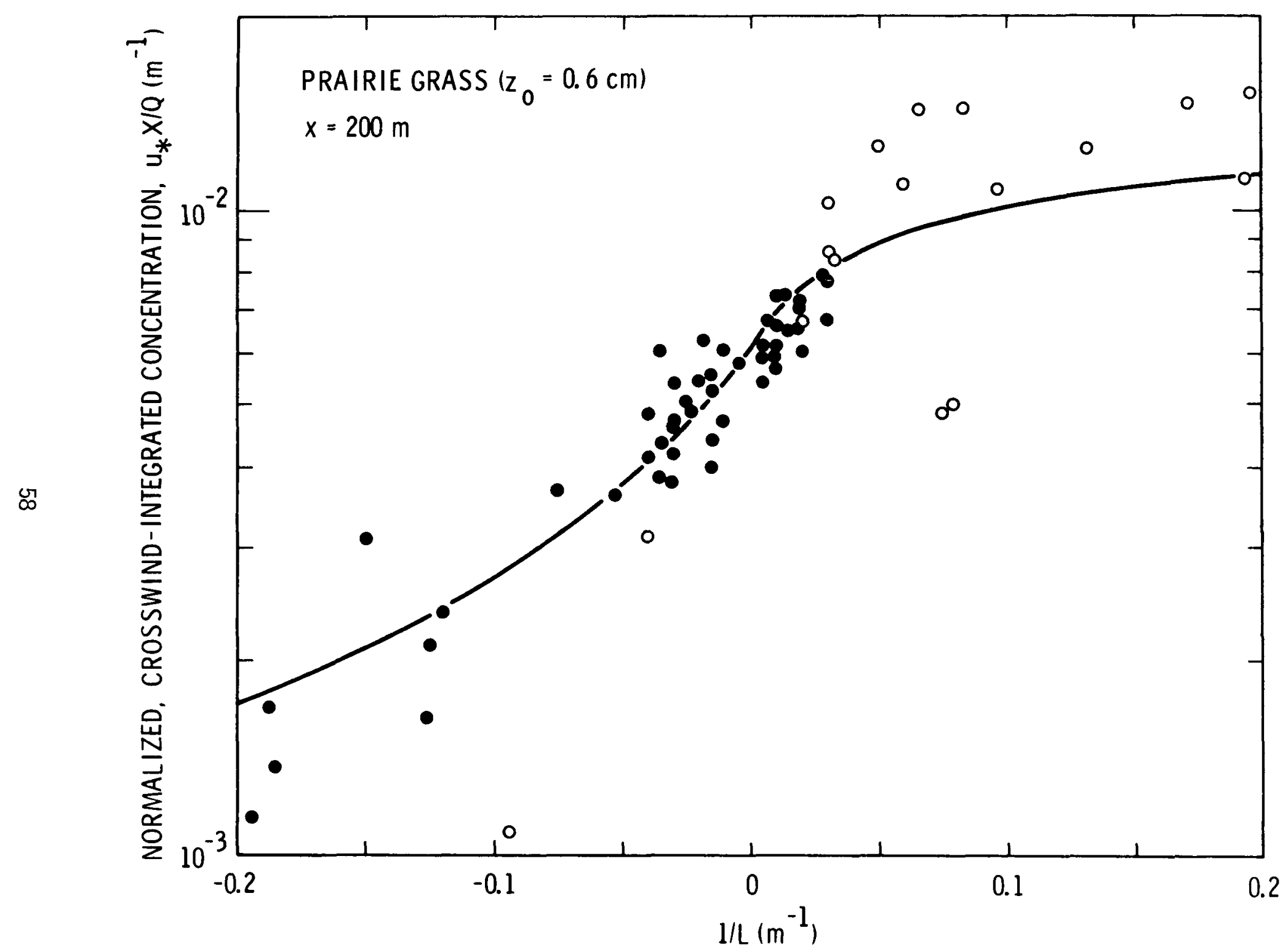

Figure 5.4. Predicted and measured CWIC $200 \mathrm{~m}$ downwind of the source as a function of atmospheric stability, Project Prairie Grass. 


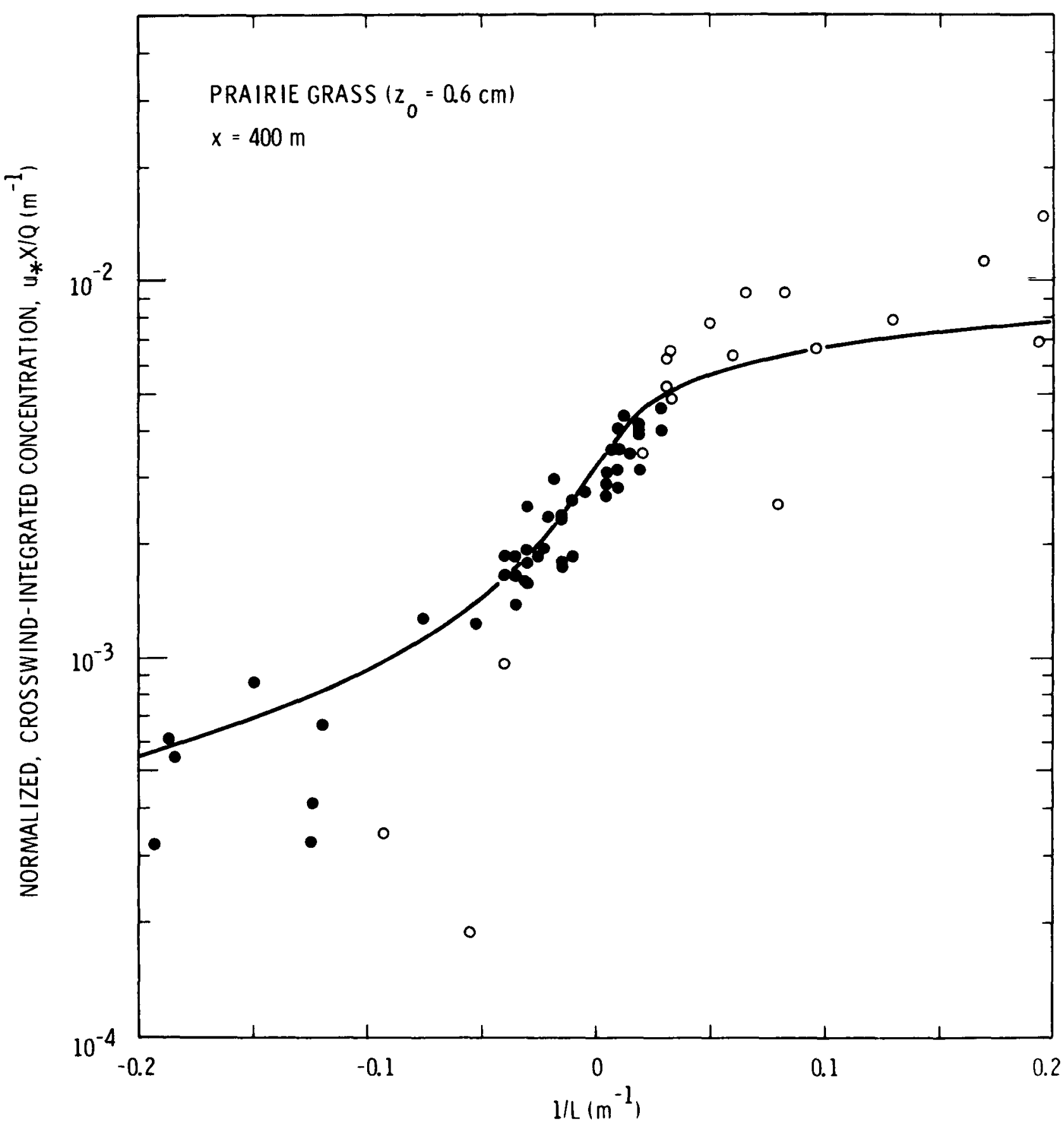

Figure 5.5. Predicted and measured CWIC $400 \mathrm{~m}$ downwind of the source as a function of atmospheric stability, Project Prairie Grass. 


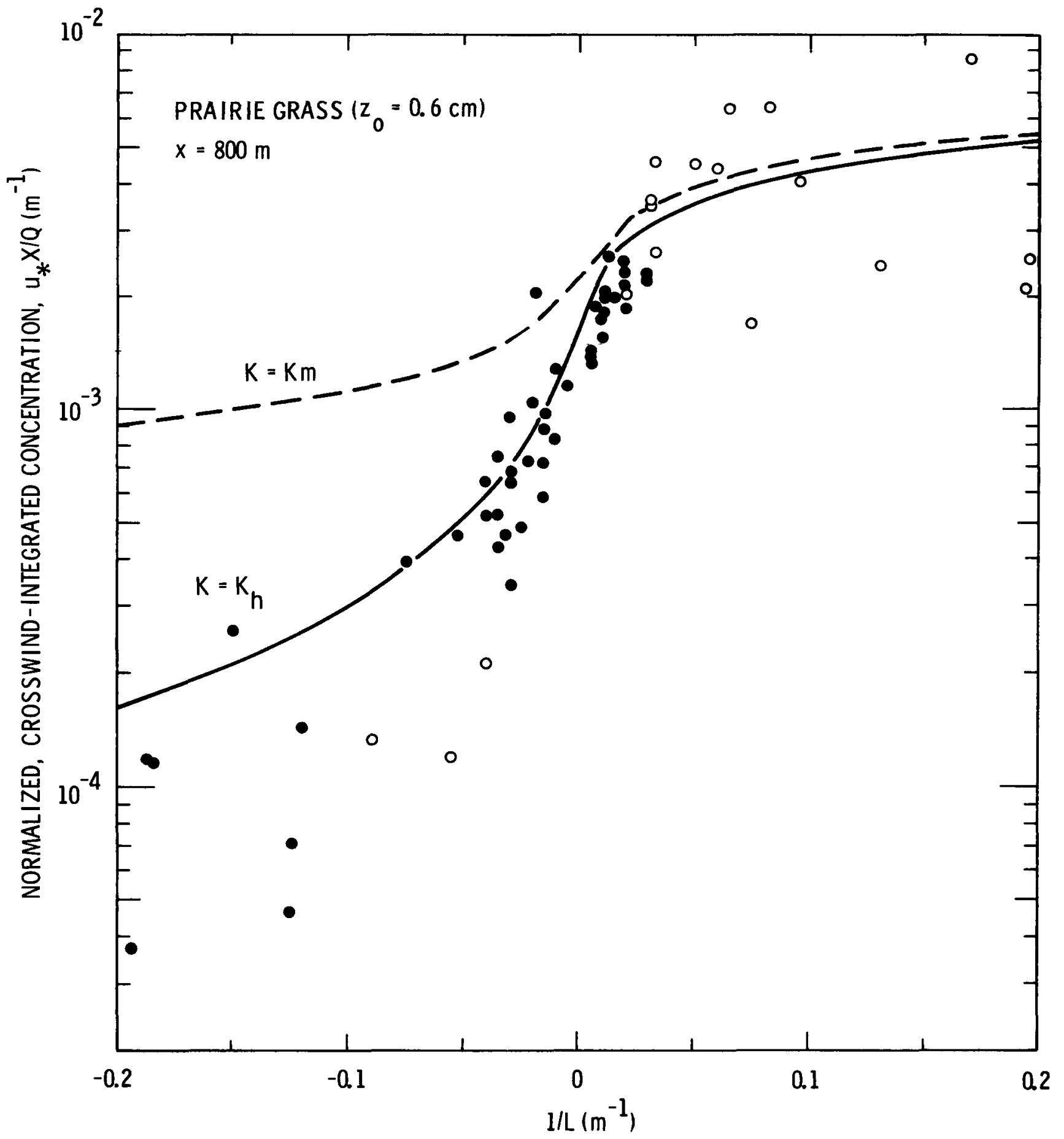

Figure 5.6. Predicted and measured CWIC $800 \mathrm{~m}$ downwind of the source as a function of atmospheric stability, Project Prairie Grass. 


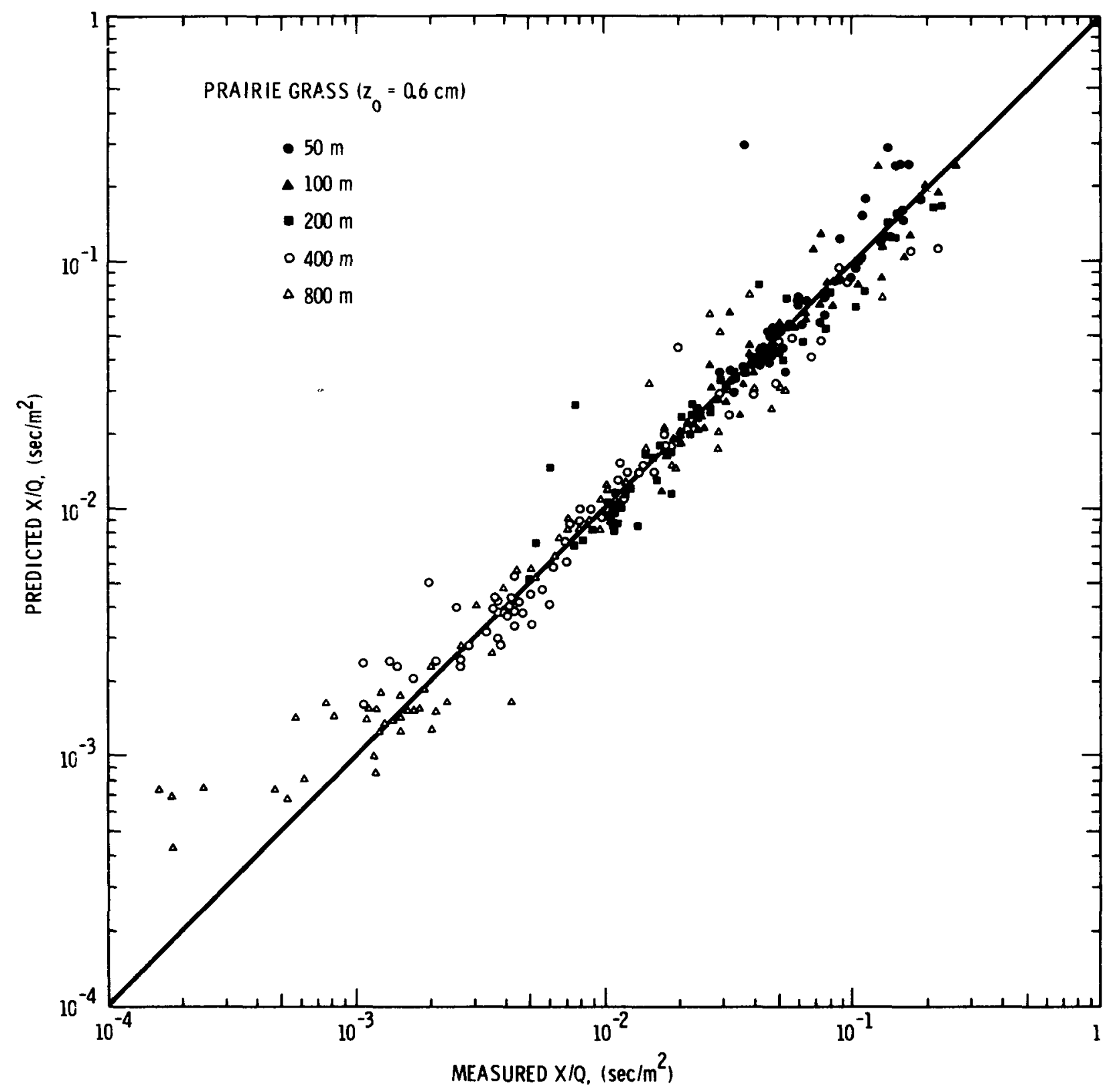

Figure 5.7. Predicted versus measured CWIC for Project Prairie Grass. 
6 cases, all at $x=800 \mathrm{~m}$ and with $x / Q<10^{-3} \mathrm{sec} / \mathrm{m}^{2}$, the measured CWIC may be too low because the individual samples are near the resolution limit of the experimental technique.

The fractional error, defined as

$$
F E \equiv \frac{[\chi / Q(\text { predicted })-\chi / Q(\text { measured })]}{\frac{1}{2}[\chi / Q(\text { predicted })+\chi / Q(\text { measured })]},
$$

has been calculated for all observations. This quantity is logarithmically unbiased, i. e. a predicted value which is $n$ times the measured value produces the same fractional error as a predicted value which is $1 / n$ of the measured value. Table 5.1 shows the mean FE and the root-mean-square FE as a function of downwind distance and Pasquill stability class. As already noted, the mean $F E$ shows $x / Q$ to be underestimated for short downwind distances and stable conditions and to be overestimated for larger distances and unstable conditions. The largest values are found for Pasquili classes $A$ and $B$ at $800 \mathrm{~m}$. These averages are dominated by the measurements mentioned above which may be too low. (A single observation dominates the FE for $50 \mathrm{~m}$ and Pasquill class F.) The r.m.s. FE is

Table 5.1. Distribution of the Fractional Error Pasquill Stability Class

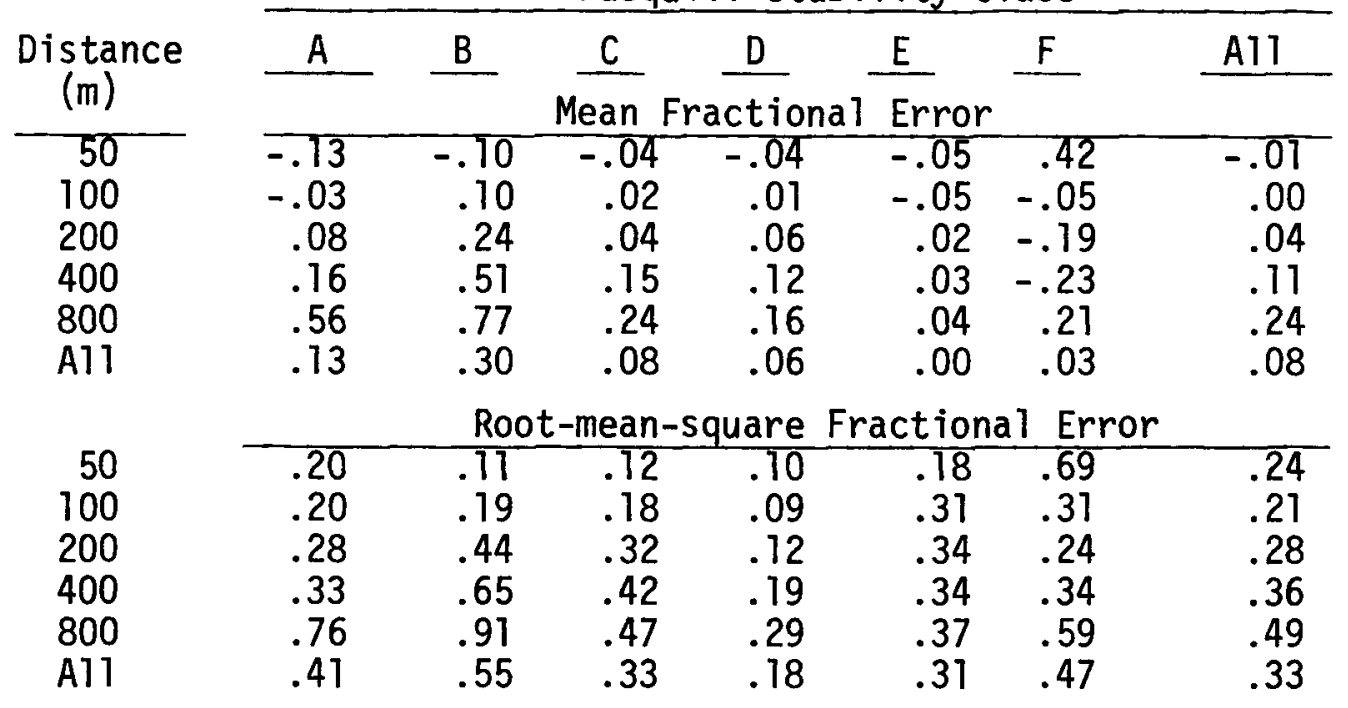


least, about .10, for neutral stability and short distances. The mean FE for all categories is .08 and the overall r.m.s. FE is .33, encouragingly small values.

The theoretical predictions are based on the assumption that the eddy diffusivity of matter is equal to that for heat. The prediction resulting from the assumption that $K$ is equal to that of momentum is shown by the broken curve in Fig. 5.6. A similar result is found at all distances. While the two predictions are little different for strongly stable conditions, the superiority of $K_{h}$ is quite obvious for near-neutral and unstable atmospheric conditions.

The predicted values of $u_{\star} \times / Q$ are not nearly as sensitive to the value of $r$. Calculations were also made with $r=1$ and $r=2$, and $r=2$ actually gave a slightly better match between theory and data than $r=1.5$ (mean FE= .02 , r.m.s. $F E=.32$ ). A value of $r=1$ gave the poorest fit to the data. At a downwind distance of $800 \mathrm{~m}, r=1$ predicts values of $u_{\star} \times / Q 10 \%$ to $35 \%$ greater than those for $r=1.5$, and $r=2$ predicts values $10 \%$ to 15\% 1ess. These differences are less at smaller distances and the ordering of the predictions becomes a function of stability.

Chatwin (1968) found that for $r=1$ the value of the constant $c$ for neutral stability is .56 . The present calculations show that for $r=1.5$ and $r=2$ the values of $c$ for neutral stability are .63 and .66 , respectively. In diabatic conditions $c$ is also a function of downwind distance and stability. For $r=1.5$ and $1 / \mathrm{L}=-0.2 \mathrm{~m}^{-1}, \mathrm{c}$ ranges from .55 at $50 \mathrm{~m}$ to .52 at $800 \mathrm{~m}$ and for $1 / \mathrm{L}=0.2 \mathrm{~m}^{-1} \mathrm{c}$ ranges from .78 at $50 \mathrm{~m}$ to .91 at $800 \mathrm{~m}$. Using a power law representation of the eddy diffusivity, Chaudhry and Meroney calculated that the error introduced by using $c=1$ was significant only near the point of release. The present calculations show that by using $c=1$ in the distance range from $50 \mathrm{~m}$ to $800 \mathrm{~m}, u_{\star} \times / Q$ is overestimated by only $5 \%$ to $10 \%$ for neutral to unstable conditions, with even smaller errors for stable conditions.

Finally, calculations were also made using Dyer's (1974) formulas for $\phi_{h}$ and $\phi_{m}$ and $k=0.41$. The r.m.s. FE for all categories, .34, is 
virtually the same as for Businger et al's formulas and the mean $\mathrm{FE}$ is -.07 .

5.2.4 Comparison with NRTS and Hanford Observations

Diffusion measurements for a ground-level source have also beer made at NRTS and Hanford, sites with rougher terrain than that used for Prairie Grass. The measurements at NRTS and the majority of those at Hanford extend to $3200 \mathrm{~m}$ downwind of the source. It is interesting to note that in similarity coordinates a four-fold gain in distance has not been realized. The largest value of $x / z_{0}$ is $1.3 \cdot 10^{5}$ for Prairie Grass, 2.1.105 for NRTS and $1.1 \cdot 10^{5}$ for Hanford. During the Green Glow experiment, measurements were also made at Hanford to $25.6 \mathrm{~km}$, or $x / z_{0}=8.5 \cdot 10^{5}$, but these were for stable conditions only.

In Figs 5.8-5.10 the observed $u_{\star} \times / Q$ are plotted as a function of $1 / L$ for the NRTS data at $200 \mathrm{~m}, 800 \mathrm{~m}$ and $3200 \mathrm{~m}$ downwind of the source, along with the theoretical predictions from Fig. 5.1. The graphs for $100 \mathrm{~m}, 400 \mathrm{~m}$, and $1600 \mathrm{~m}$ are quite similar. There are fewer data than for the Prairie Grass experiments and the scatter is greater, but the NRTS data also support the Lagrangian similarity prediction of the groundlevel contaminant concentrations.

For the Hanford Green Glow-30 Series data, the observed values of $u_{\star} x\left(x_{3} z=1.5 \mathrm{~m}\right) / Q$ were found to be generally a factor of two smaller than the predicted values. Since this reduction is constant beyond a downwind distance of $200 \mathrm{~m}$, it can be explained by a large loss of tracer prior to the $200 \mathrm{~m}$ arc. This may be due either to dry deposition in the first $200 \mathrm{~m}$ or to depletion of the tracer in the immediate vicinity of the source. This interpretation is supported by the agreement between measured (Weber et al, 1977 ) and predicted values of $\sigma_{z}$ at $200 \mathrm{~m}$ shown in Fig. 5.11 and is also consistent with mass balance computations made by Simpson (1961). Therefore the Hanford data were plotted as $x(x, z=1.5 \mathrm{~m}) / x(x=200 \mathrm{~m}, z=1.5 \mathrm{~m})$.

$x(x) / x(200 m)$ is plotted in Fig. 5.12-5.14 as a function of $1 / \mathrm{L}$ for the $800 \mathrm{~m}, 3200 \mathrm{~m}$ and $12.8 \mathrm{~km}$ Hanford data, along with the theoretical predictions based on Fig. 5.1. The $800 \mathrm{~m}$ and $3200 \mathrm{~m}$ data, and similar data 


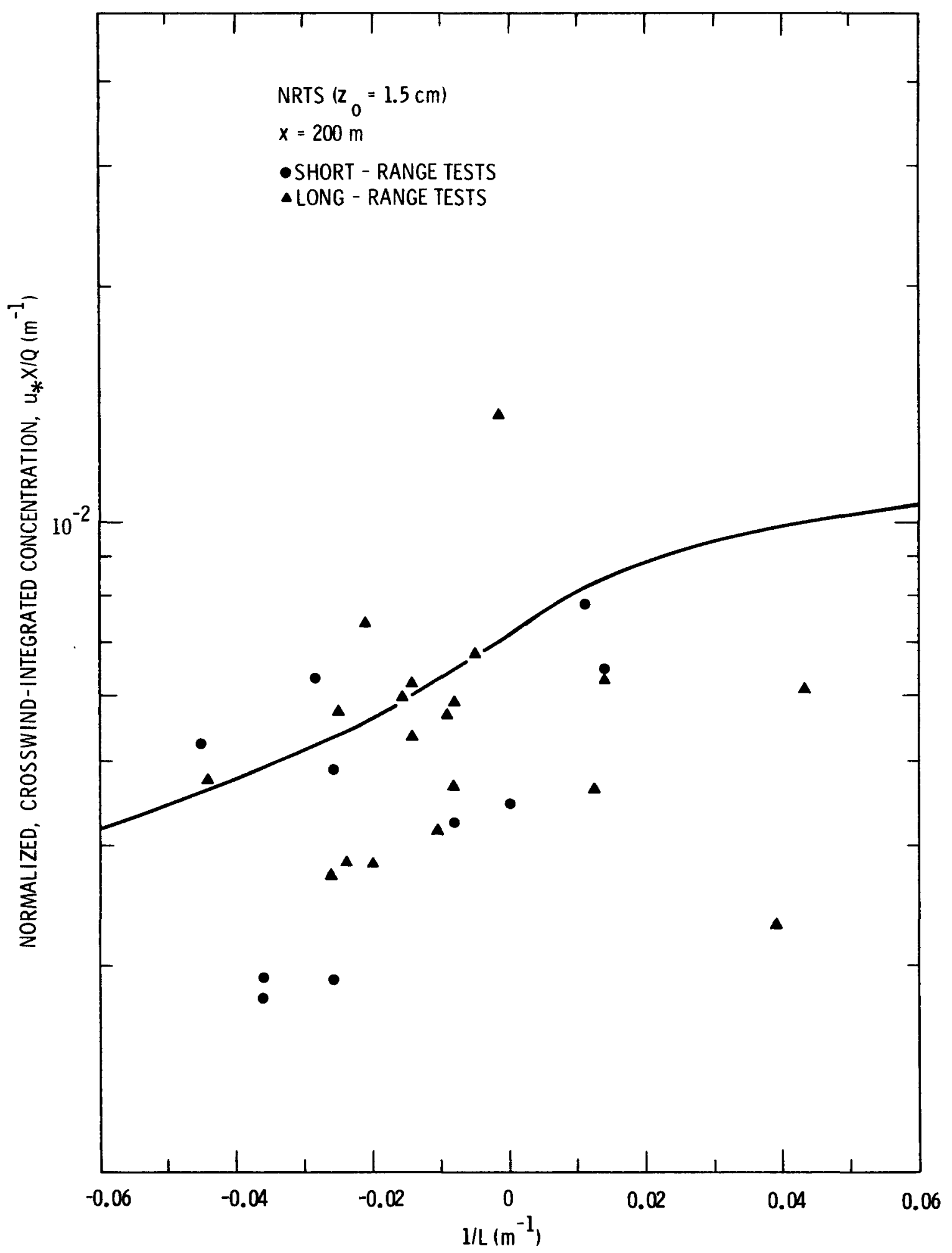

Figure 5.8. Predicted and measured CWIC $200 \mathrm{~m}$ downwind of the source as a function of atmospheric stability, NRTS. 


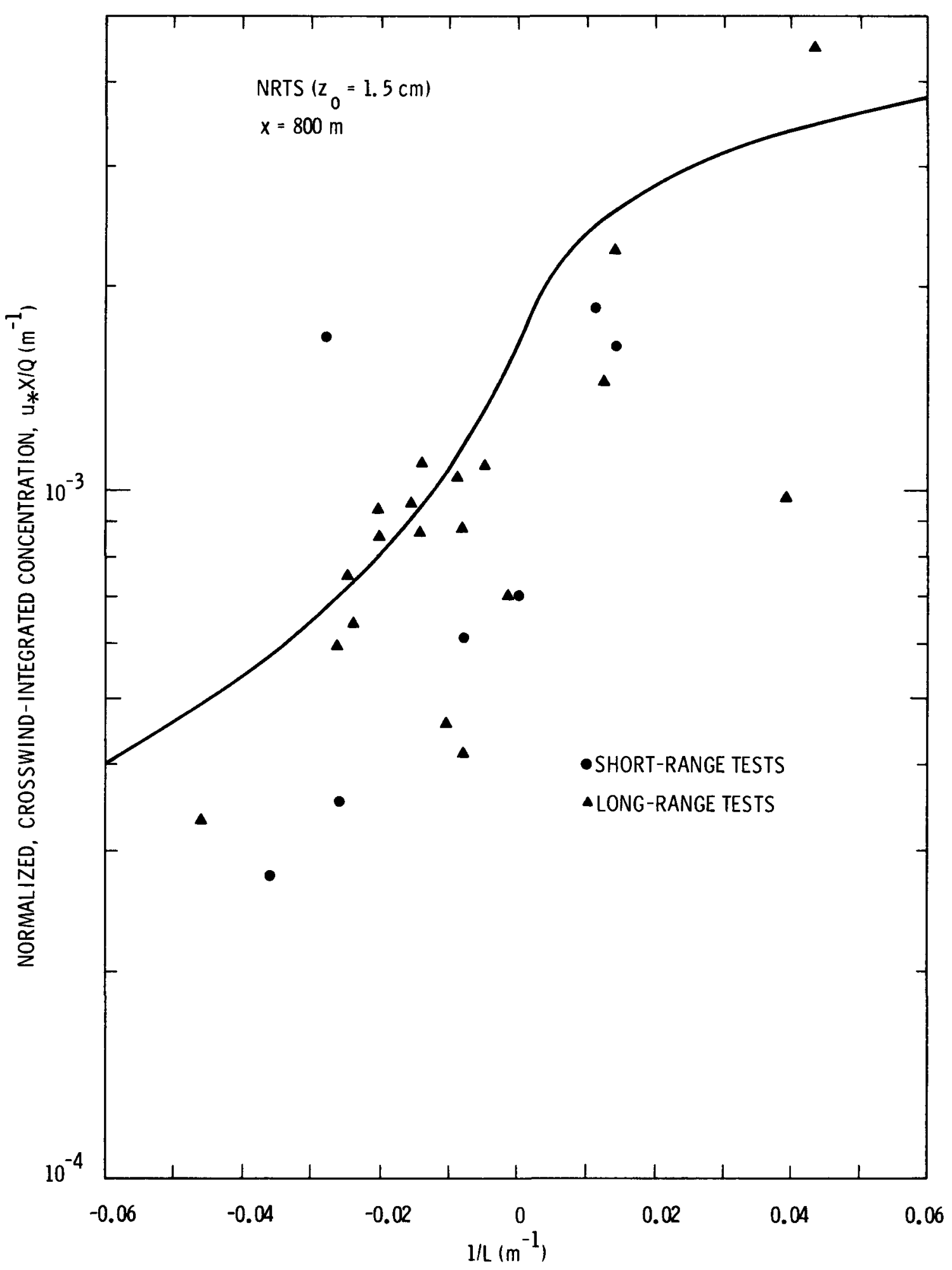

Figure 5.9. Predicted and measured CWIC $800 \mathrm{~m}$ downwind of the source as a function of atmospheric stability, NRTS. 


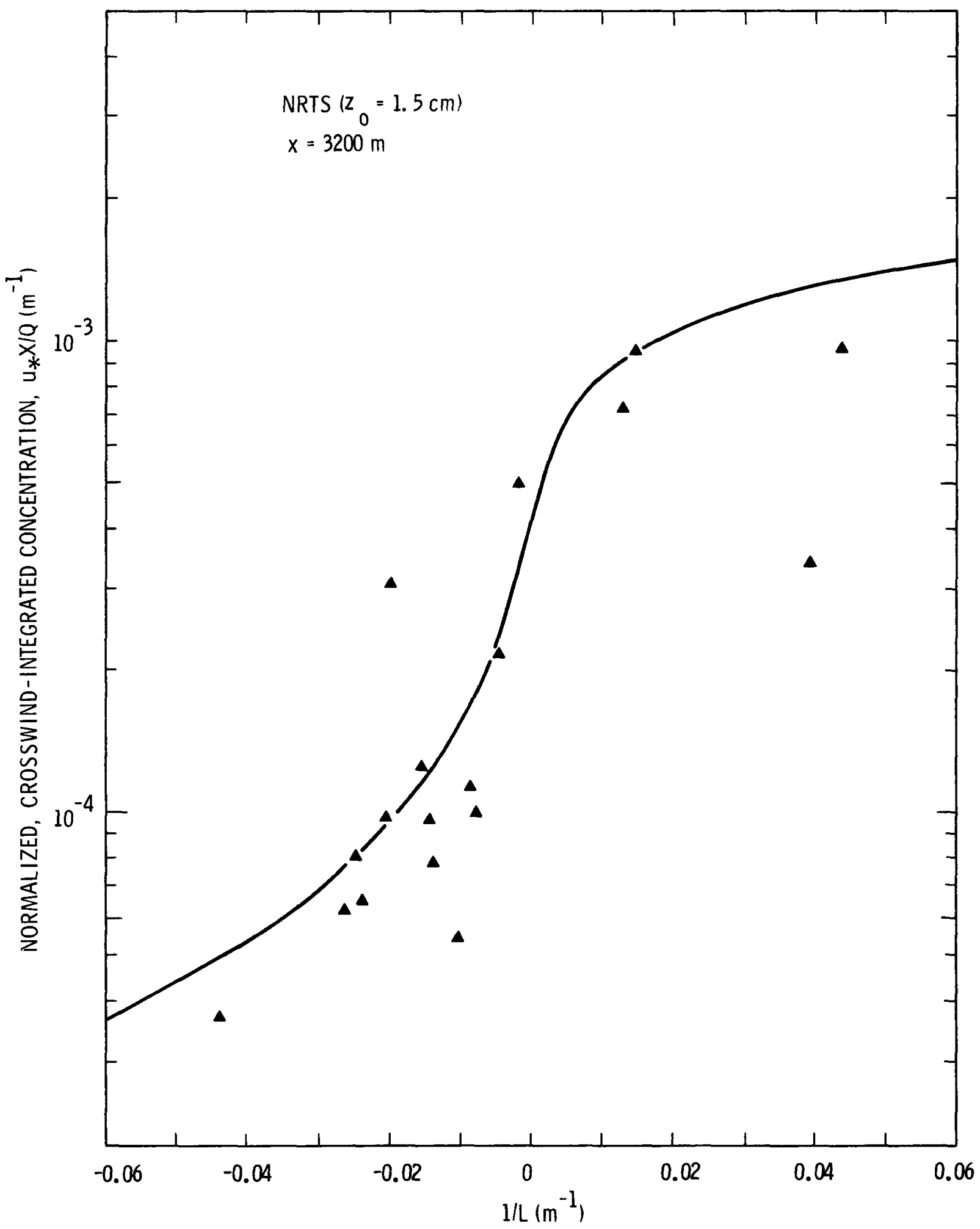

Figure 5.10. Predicted and measured CWIC $3200 \mathrm{~m}$ downwind of the source as a function of atmospheric stability, NRTS. 


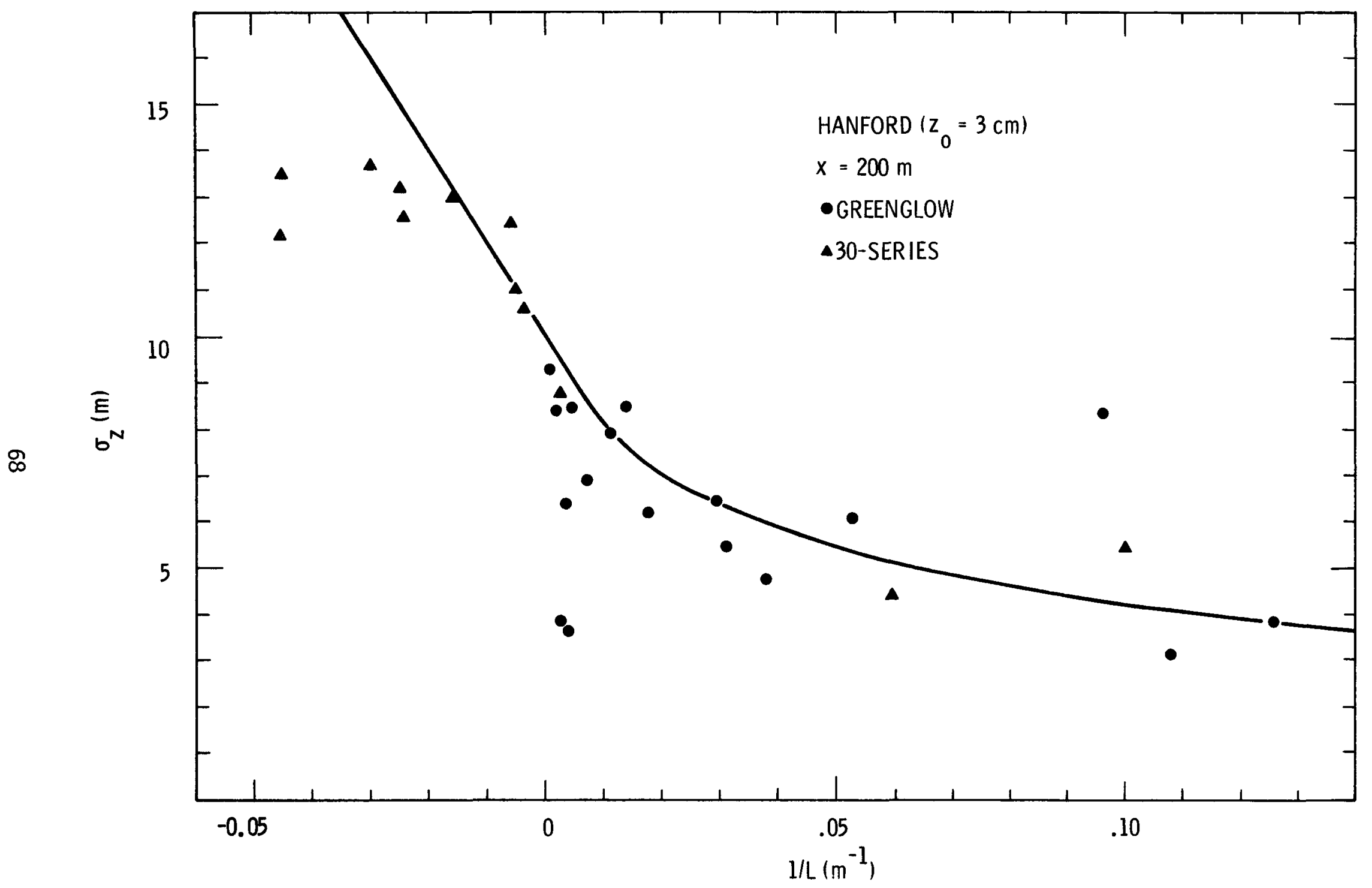

Figure 5.11. $\sigma_{z} 200 \mathrm{~m}$ downwind of the source as a function of atmospheric stability, Green Glow-30 Series. 


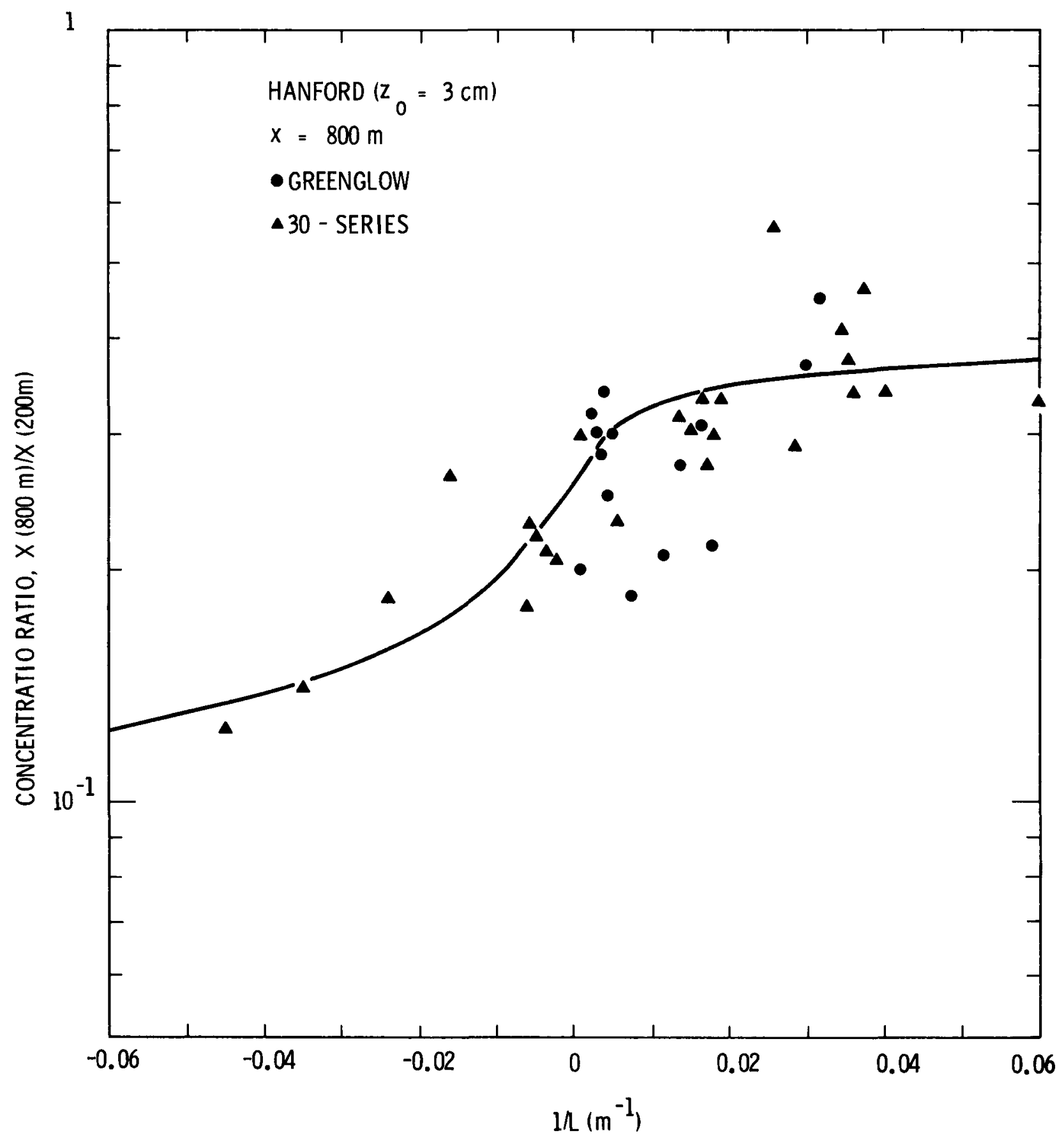

Figure 5.12. Predicted and measured ratio of CWIC at $800 \mathrm{~m}$ to that at $200 \mathrm{~m}$ as a function of atmospheric stability, Green Glow-30 Series. 


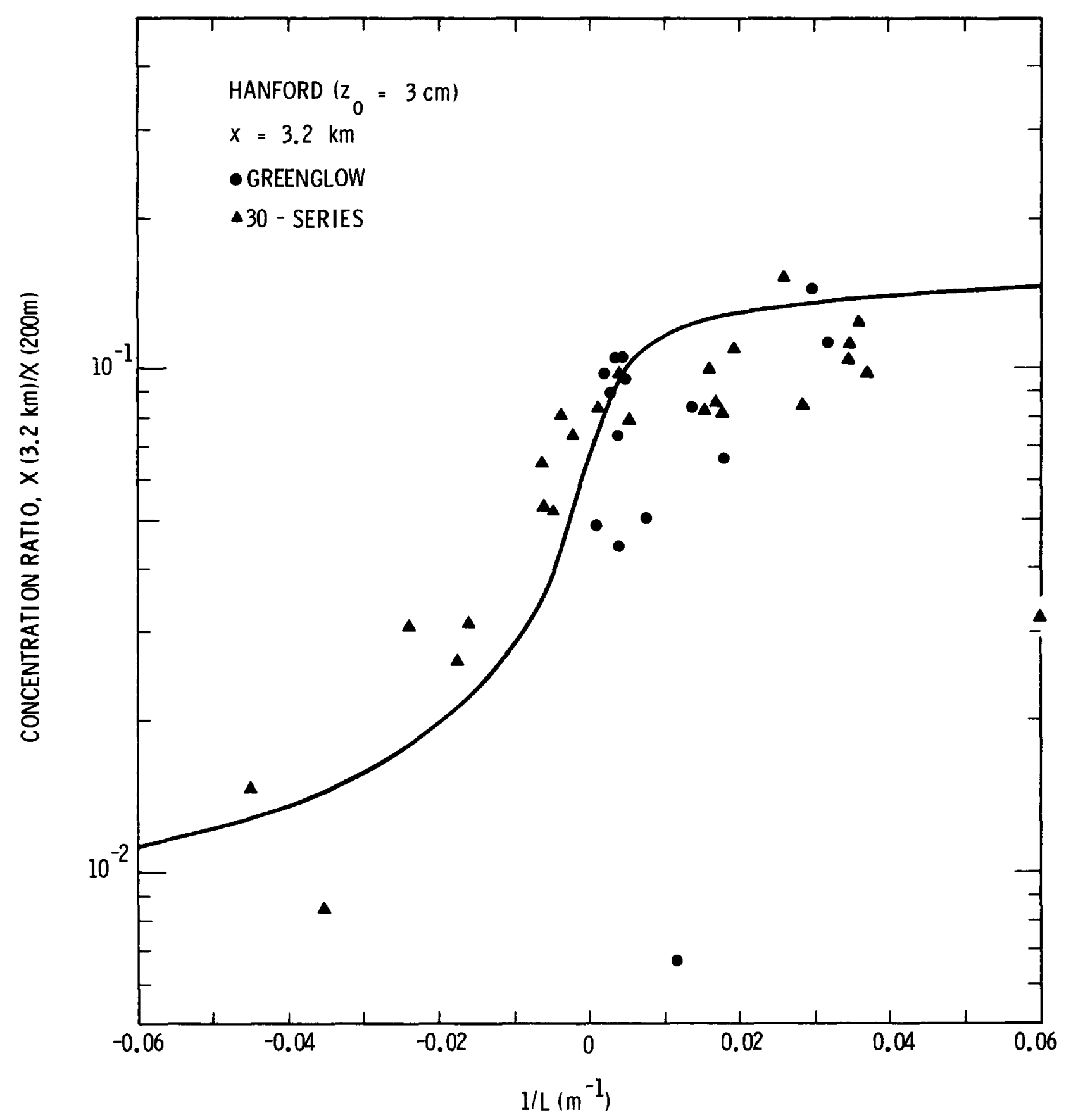

Figure 5.13. Predicted and measured ratio of CWIC at $3.2 \mathrm{~km}$ to that at $200 \mathrm{~m}$ as a function of atmospheric stability, Green Glow-30 Series. 


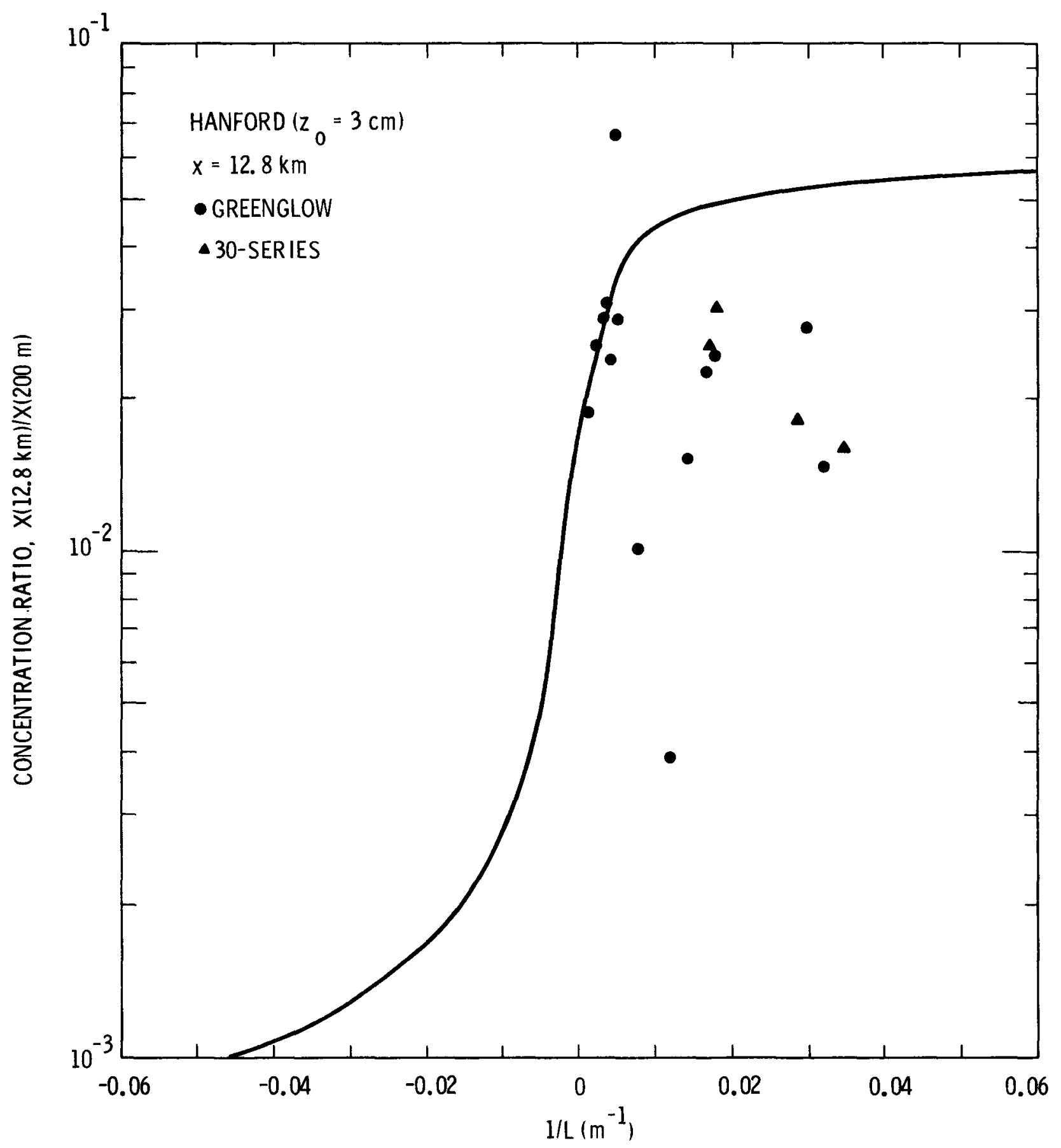

Figure 5.14. Predicted and measured ratio of CWIC at $12.8 \mathrm{~km}$ to that at $200 \mathrm{~m}$ as a function of atmospheric stability, Green Glow-30 Series. 
at $1600 \mathrm{~m}$, contain a large amount of scatter but support the Lagrangian similarity predictions. An exception is the tendency for the $3200 \mathrm{~m}$ observations to fall below the theoretical curve during stable conditions. This discrepancy increases with downwind distance and is especially obvious at $12.8 \mathrm{~km}$ where only the near-neutral data match the predictions. One explanation for the apparent loss of tracer may be dry deposition, since the depletion of a plume from a ground-level source is greatest during stable conditions (Horst, 1977).

\subsubsection{Discussion and Application}

This comparison of the observed CWIC with that predicted by Lagrangian similarity theory provides support for the theory and tests the assumptions that (1) the eddy diffusivity of passive contaminants corresponds to that of heat rather than that of momentum and (2) the effect of atmospheric stability on the vertical distribution is to alter the scale of the diffusion $\bar{z}$ without strongly affecting the form of the distribution. The data leave little question about the validity of the first assumption, especially for unstable conditions where the diffusivities differ the most. However, the degree to which the form of the vertical distribution depends on stability is still open to question.

Direct measurements of the vertical distribution lead to the choice of $r=1.5$ in (5.7). Although this value gives the best fit to the Prairie Grass ground-level concentration data at $50 \mathrm{~m}$ to $200 \mathrm{~m}$, the data at $400 \mathrm{~m}$ and $800 \mathrm{~m}$ fall below the theoretical curve and $r=2$ gives a better fit. A similar trend is observed in the NRTS data. Since the effect of atmospheric stability on diffusion increases with downwind distance, this discrepancy may reflect changes in the form of the vertical distribution. A decrease in ground-level concentration below that predicted by (5.7) is consistent with the observations of Deardorff and Willis (1975) and Lewellen and Teske (1976) that the height of the maximum concentration increases with downwind distance in an unstable atmosphere. However, the discrepancy could also be due to inadequacy of the empirical forms for $\phi_{h}$ and $\phi_{m}$ as the diffusion proceeds beyond the surface flux layer. When the 
plume extends beyond this layer, additional parameters such as the depth of the mixed layer also influence the diffusion process. A third explanation would be dry deposition of the $\mathrm{SO}_{2}$ tracer. No attempt has been made to distinguish among these possibilities.

The Prairie Grass data are for a surface roughness of $z_{0}=0.6 \mathrm{~cm}$. The NRTS data for $z_{0}=1.5 \mathrm{~cm}$ and, to a lesser degree, the Hanford data for $z_{0}=3 \mathrm{~cm}$ demonstrate the validity of the predicted dependence on surface roughness and extend the validation to greater downwind distances. The NRTS data at $1600 \mathrm{~m}$ and $3200 \mathrm{~m}$, or $x / z_{0}=1.1 \cdot 10^{5}$ and $2.1 \cdot 10^{5}$, appear to fall below the theoretical curve in a manner similar to that of the Prairie Grass data at $800 \mathrm{~m}$ where $x / z_{0}=1.3 \cdot 10^{5}$. The theory is also substantiated by Hanford data from $200 \mathrm{~m}$ to $3200 \mathrm{~m}, \mathrm{x} / \mathrm{z}_{\mathrm{o}} \leq 1.1 \cdot 10^{5}$, although the ratio of air concentrations at two downwind distances is a less demanding test. The Hanford data at 12.8 and $25.6 \mathrm{~km}, x / z_{0}=2.6 \cdot 10^{5}$ and $8.5 \cdot 10^{5}$, support the theory only for near-neutral conditions. The apparent loss of tracer at these distances under stable conditions could be due to any of the reasons 1 isted in the previous paragraph.

Finally, this analysis provides a means to predict the CWIC to a dimensionless distance of $x / z_{0} \leq 2 \cdot 10^{5}$. Substituting (5.13) into (5.7)

$$
x(x, z)=\frac{A Q}{\bar{z} u(c \bar{z})} \exp \left[-(z / b \bar{z})^{r}\right] .
$$

where $A=r \Gamma(2 / r) / \Gamma^{2}(1 / r) . \quad \bar{z}$ is determined from Eq. (5.5). A value of $r=1.5$ was found to provide the best fit to the Prairie Grass data to a distance of $x / z_{0}=3 \times 10^{4}$ and hence $A=.731, b=1.52$ and $c=.63$ for neutral stability. Since the CWIC is insensitive to the value of $c$, it appears unnecessary to account for its change during diabatic conditions.

Since the CWIC is also insensitive to the value of $r$ and $r=2$ actually gives the best overall fit to the Prairie Grass data, one could as well use the familiar Gaussian form,

$$
x(x, z)=\frac{\sqrt{2 / \pi} Q}{\sigma_{z} u\left(c^{\prime} \sigma_{z}\right)} \exp \left[-z^{2} / 2 \sigma_{z}{ }^{2}\right] .
$$


Here $c^{\prime}=.53$ for neutral stability since $c=.66$ and $\sigma_{z}=\sqrt{\pi / 2} \bar{z}$ when $r=2$. The one difference from the conventional formulation is that here the height for the measurement of the wind speed is specified as an implicit function of downwind distance.

\subsection{ELEVATED SOURCES: HANFORD 67-SERIES}

\subsubsection{Concentration Profiles from Tower Data}

During the Hanford 67-Series, tower samplers were activated in 14 elevated release tests, but in only eight of these were sufficient data collected to allow a reasonable analys is of the vertical dispersion. These tests were $V 1$ through V8, and featured up to three tracers ( $\mathrm{ZnS}$, rhodamine and $\mathrm{Kr}^{85}$ ) simultaneously released from a height of $26 \mathrm{~m}$. Crosswind arrays of five towers were usually instrumented with samplers at downwind distances of $200,800,1600$ and $3200 \mathrm{~m}$. A vertical profile, with a peak value of unity, was computed from the tower data at each distance. Details of this analysis are presented in Section 3.4.

The most commoniy used expression for the spatial distribution of a pollutant diffusing from an elevated source is the Gaussian formula (4.1). Integrating this expression in the crosswind direction to el iminate any dependence on $y$,

$$
\begin{gathered}
x(x, z)=\int_{-\infty}^{\infty} x(x, y, z) d y \\
=\frac{Q}{\sqrt{2 \pi u \sigma_{z}}}\left\{\exp \left[-\frac{(z-h)^{2}}{2 \sigma_{z}{ }^{2}}\right]+\exp \left[-\frac{(z+h)^{2}}{2 \sigma_{z}^{2}}\right]\right\} .
\end{gathered}
$$

As $\sigma_{z}$ increases and becomes comparable to $h$, it might be anticipated that (5.17) would no longer be an adequate description of the observed profiles. Vertical gradients of the wind speed and diffusivity, as well as deposition to the surface, would be expected to produce significant deviations from this simple form. These effects should become more pro- 
nounced as the downwind distance increases.

The experimental results confirm these expectations. At $200 \mathrm{~m}$ downstream, the observed profiles can be fit reasonably well with the expression given in (5.17), normalized by its peak value. A value of $\sigma_{z}$ was chosen to provide the "best fit" of (5.17) to the experimental values. The criterion used was that the sum of the squares of the differences between measured and calculated concentration values be a minimum, but in view of the irregularities of the profiles, $\sigma_{z}$ was determined only to the nearest meter. Fig. 5.15 shows a particularly good fit to the data, while Fig. 5.16 shows the worst agreement achieved for the $200 \mathrm{~m}$ arc. Nonetheless, the fit is adequate.

Since up to three tracers were released simultaneously from the same height, it is possible to obtain independent determinations of $\sigma_{z}$ for the same meteorological conditions. The $200 \mathrm{~m}$ arc results are shown in Table 5.2 and the agreement is seen to be generally quite good. (Also shown in Table 5.2 are $\sigma_{z}$ values determined from the CWIC. These are discussed in the following section.)

Table 5.2. $\sigma_{z}$ Values at $x=200 \mathrm{~m}$ for $26 \mathrm{~m}$ releases

$$
{ }_{-}^{\sigma} \text { from tower profiles } \sigma_{z} \text { from CWIC }
$$

\begin{tabular}{|c|c|c|c|c|c|c|}
\hline Test & $1 / L\left(m^{-1}\right)$ & $\mathrm{ZnS}$ & $\mathrm{kr}^{85}$ & $\mathrm{R}$ & $\mathrm{ZnS}$ & $\mathrm{R}$ \\
\hline V1 & -.005 & 12 & - & 12 & 9 & 8 \\
\hline V2 & & 13 & 12 & 11 & 11 & 9 \\
\hline V3 & -.003 & 15 & - & 19 & $=$ & - \\
\hline V4 & -.003 & 8 & - & 8 & 7 & - \\
\hline V5 & .067 & 9 & 9 & 7 & 8 & 7 \\
\hline V6 & .020 & 11 & 8 & - & 9 & 7 \\
\hline V7 & -.097 & 16 & 17 & - & 12 & - \\
\hline V8 & .008 & 10 & - & - & 13 & . \\
\hline
\end{tabular}

At $800 \mathrm{~m}$ downstream, however, the average root-mean-square error for the $\mathrm{ZnS}$ profiles is nearly twice as great as that found at $200 \mathrm{~m}$. The applicability of $(5.17)$ to describe the vertical concentration profiles is thus questionable at such distances. Fig. 5.17 shows an example of the poor fit obtained at $800 \mathrm{~m}$; the data are from the same test (V6) as shown in Fig. 5.15. 


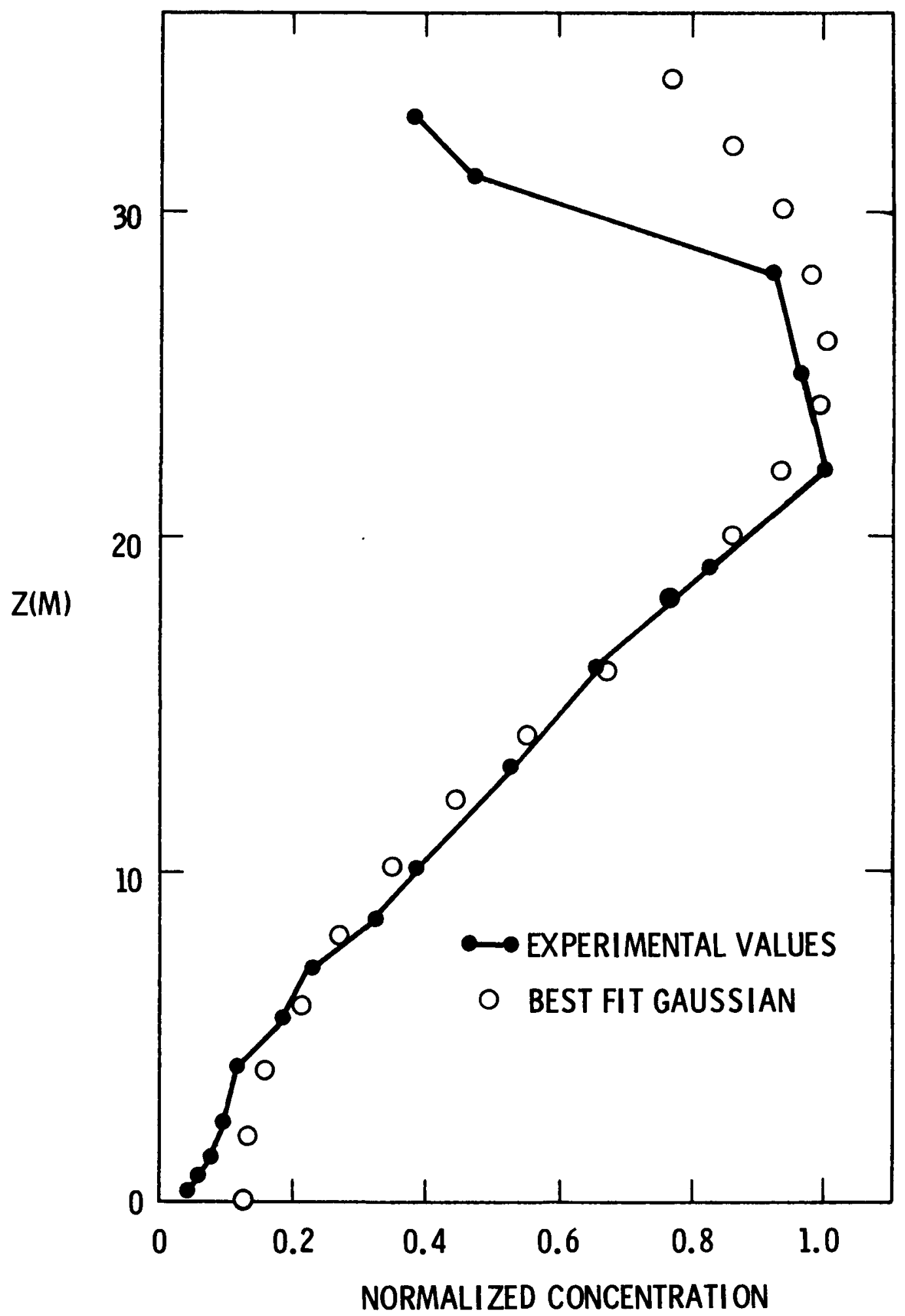

Figure 5.15. Comparison of measured concentration profile and best fit Gaussian at $200 \mathrm{~m}$ downwind for $26 \mathrm{~m}$ release, Test V6. 


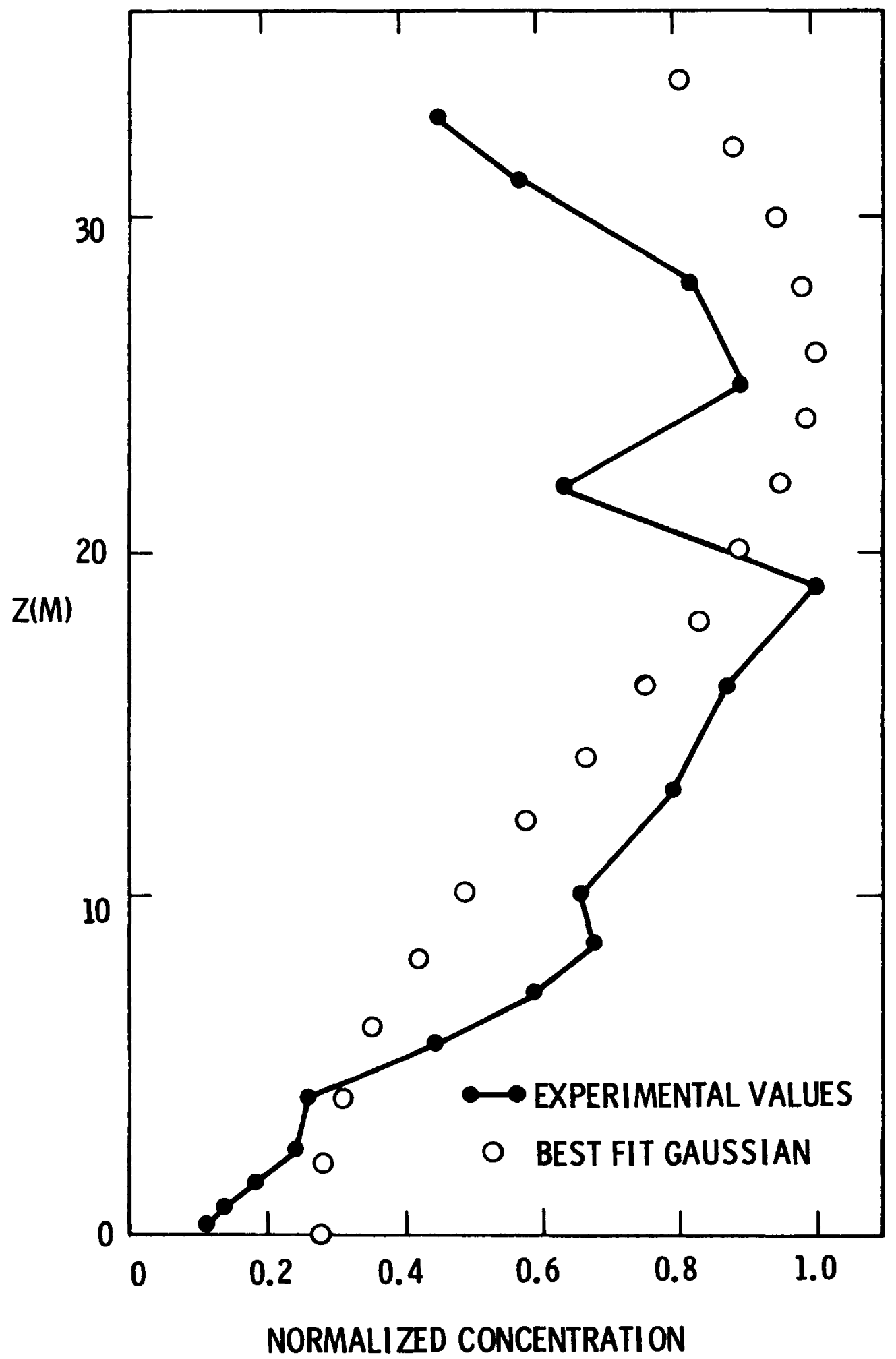

Figure 5.16. Comparison of measured concentration profile and best fit Gaussian at $200 \mathrm{~m}$ downwind for $26 \mathrm{~m}$ release, Test V2. 


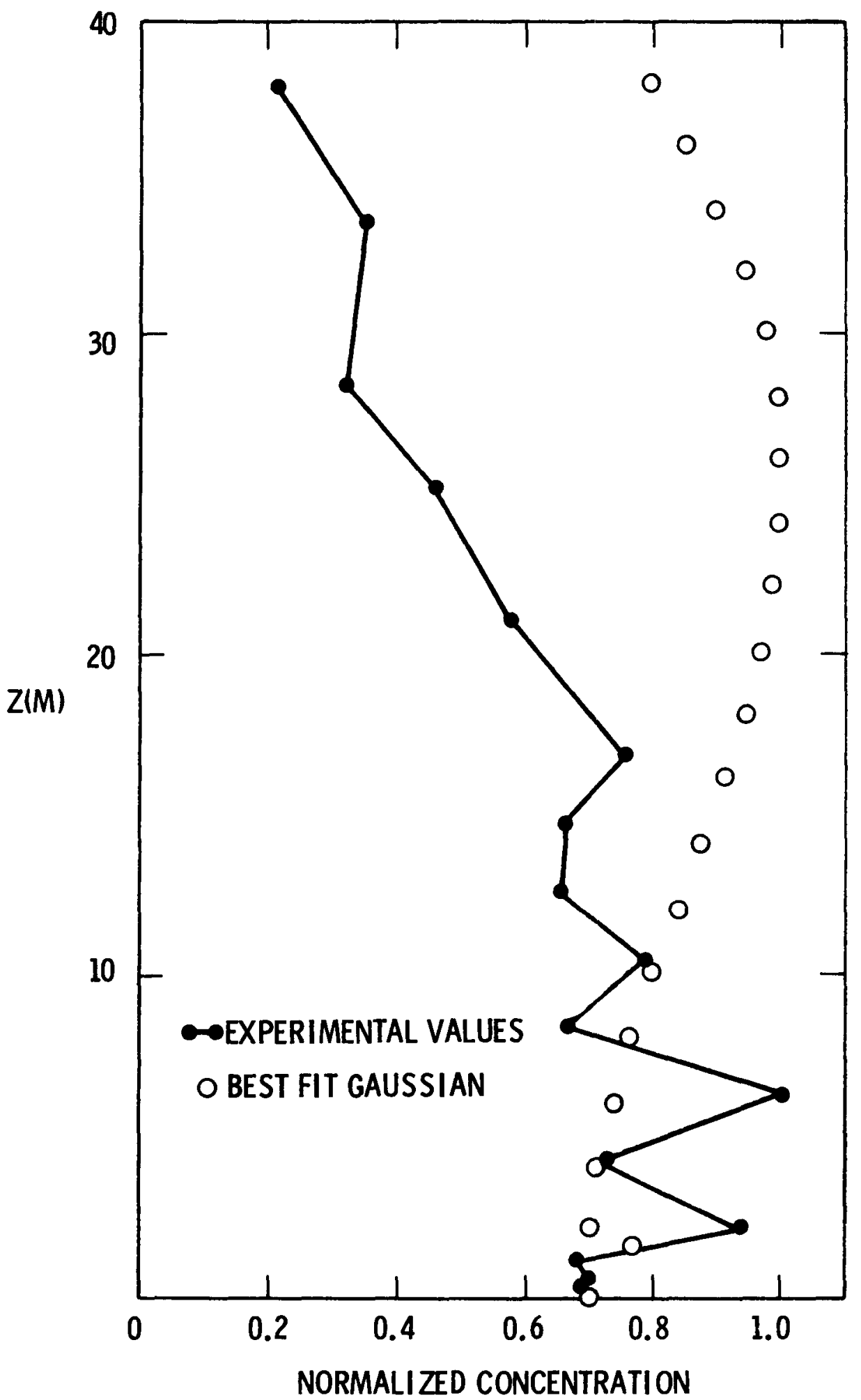

Figure 5.17. Comparison of measured concentration profile and best fit Gaussian at $800 \mathrm{~m}$ downwind for $26 \mathrm{~m}$ release, Test $V 6$. 


\subsection{2 $\sigma_{z}$ from Crosswind-Integrated Concentrations}

As mentioned in Section 5.1, $\sigma_{z}$ may also be determined from (5.17) using only the ground-level CWIC measurements. For an elevated source, (5.17) yields two roots for $\sigma_{z}$ but these simply correspond to whether $x$ is upwind or downwind of the maximum ground-level CWIC.

In the simplest Gaussian approach, the wind speed $u$ in (5.17) is constant with height. This is clearly not the case for the actual tests and an effective $u$, $u_{\text {eff }}$, may be defined by the mass conservation equation (5.9). If $u(z)$ is represented as a function of height, (5.9) and (5.17) may then be solved iteratively to obtain a consistent set of values for $u_{\text {eff }}$ and $\sigma_{z}$. In practice it was found that for the relatively short downwind distances at which (5.17) might be considered valid, $\bar{u}_{\text {eff }}$ $\approx u(h)$, the wind speed at the height of release. (5.17) was therefore used by itself to determine $\sigma_{z}$, with the mean wind speed chosen to be $u(h)$.

There is considerable evidence from ground-level-release tests that substantial fractions of a plume may be deposited as it moves downwind past the various sampling arcs (e.g., Simpson, 1951;'Islitzer and Dumbauld, 1963). Analysis of the present data leads to a similar conclusion. From $(5.17)$ the maximum ground-level CWIC should occur at a distance where $\sigma_{z}=h$ and be equal to $2 Q /(\sqrt{2 \pi e} u h)$. The measured maxima were generally lower than this theoretical value, although in a small fraction of the tests these theoretical maxima were actually exceeded. For $\sigma_{z}$ less than $h$, however, deposition may be anticipated to be relatively unimportant. Thus it may be possible to determine values of $\sigma_{z}$ from the CWIC, provided this is done only at relatively small downstream distances.

Tests $V 1$ through $V 8$ provided an opportunity to compare values of $\sigma_{z}$ obtained from the CWIC at $200 \mathrm{~m}$ downwind with those derived from tower measurements. The mean ratio of the former to the latter is $0.87 \pm .17$, 
and the individual results have been shown in Table 5.2. In all cases. $\sigma_{z} \leqslant 0.7 \mathrm{~h}$. In this range of $\sigma_{z}$, therefore, use of the CWIC to obtain $\sigma_{z}$ does not result in large errors, although there is a tendency to underestimate the presumably more representative tower profile values. For larger $\sigma_{z}$, neither approach is particularly useful. As a consequence, no results from the Hanford 67-Series elevated releases have been included here for downwind distances greater than $400 \mathrm{~m}$.

\subsubsection{Comparison with Ground-Level Source Behavior}

In Section 5.2, Lagrangian similarity theory was shown to be a reliable predictor of vertical dispersion from a ground-level source. This approach works well over a large range of stabilities and will be used here to compare the dispersion from ground-level and elevated sources.

Fig. 5.18 shows the results obtained from the tower data of tests VI through V8 at a downwind distance of $200 \mathrm{~m}$. The solid line corresponds to the ground-level release $\sigma_{z}$ predicted from (5.5). Considering the scatter of the points, the results correspond fairly closely to the Lagrangian similarity predictions in near-neutral or slightly stable conditions, but begin to deviate strongly from them as stability increases.

Fig. 5.19 shows $\sigma_{z}$ values derived from the CWIC at $400 \mathrm{~m}$ downstream for the remaining $26 \mathrm{~m}$ releases. The solid line again corresponds to the theoretical expression, and the results are seen to be similar to those in Fig. 5.18.

Fig. 5.20 shows the results obtained from an analys is of the CWIC for the $56 \mathrm{~m}$ releases. While the tendency is again an increase in $\sigma_{z}$ values over those associated with ground-level releases, it is apparent from the large scatter that $L$ alone is not sufficient for describing vertical diffusion in this region. This is not surprising, since for stable conditions the $56 \mathrm{~m}$ height of release may well lie outside the surface flux boundary layer. Moreover, there are uncertainties in the computed values of $L$, and small changes in this quantity can have a dramatic effect on the predicted values of $\sigma_{z}$. 


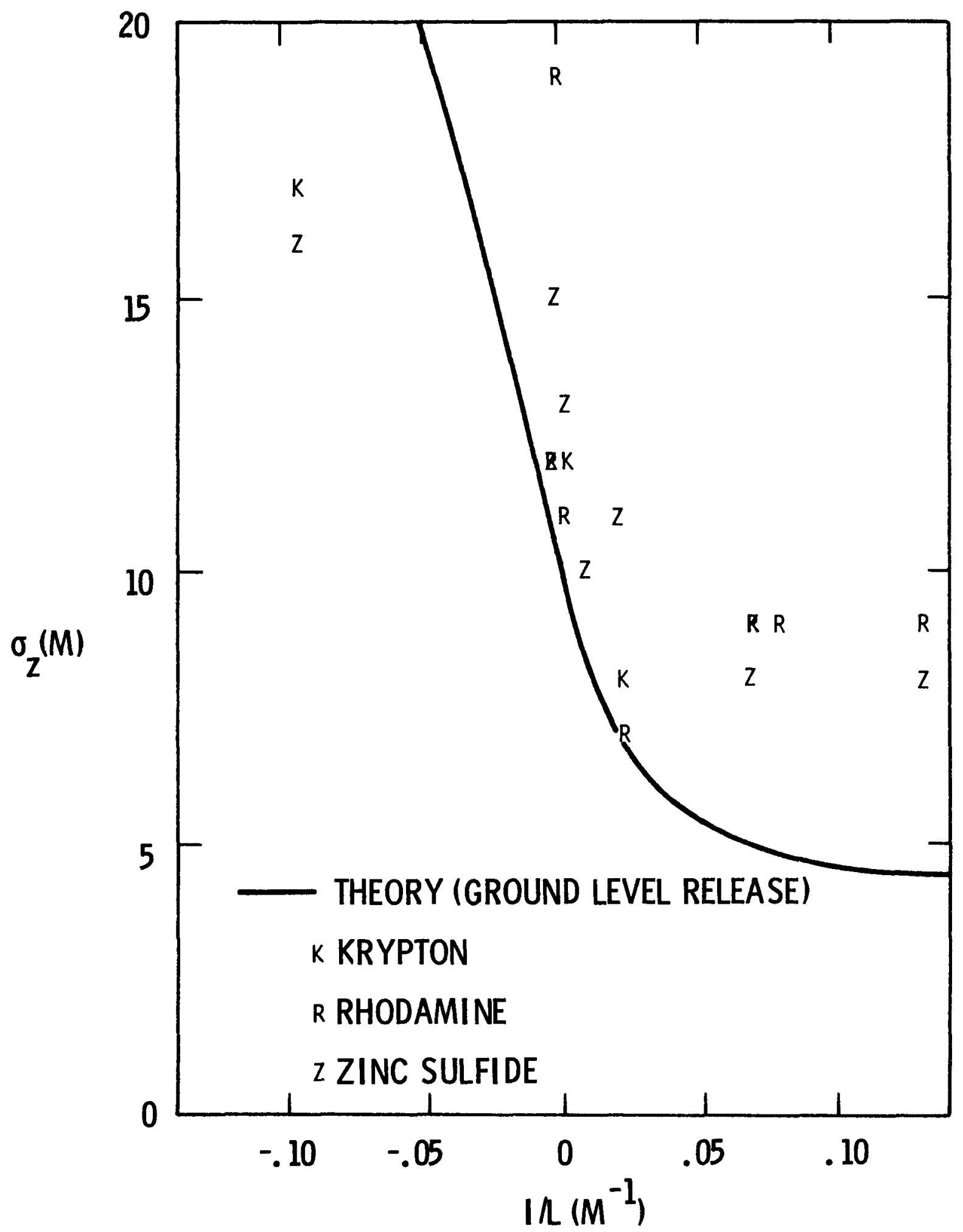

Figure 5.18. Comparison of $\sigma$ at $200 \mathrm{~m}$ downwind for $26 \mathrm{~m}$ releases with Lagrangian similarity predictions for ground-level releases. 


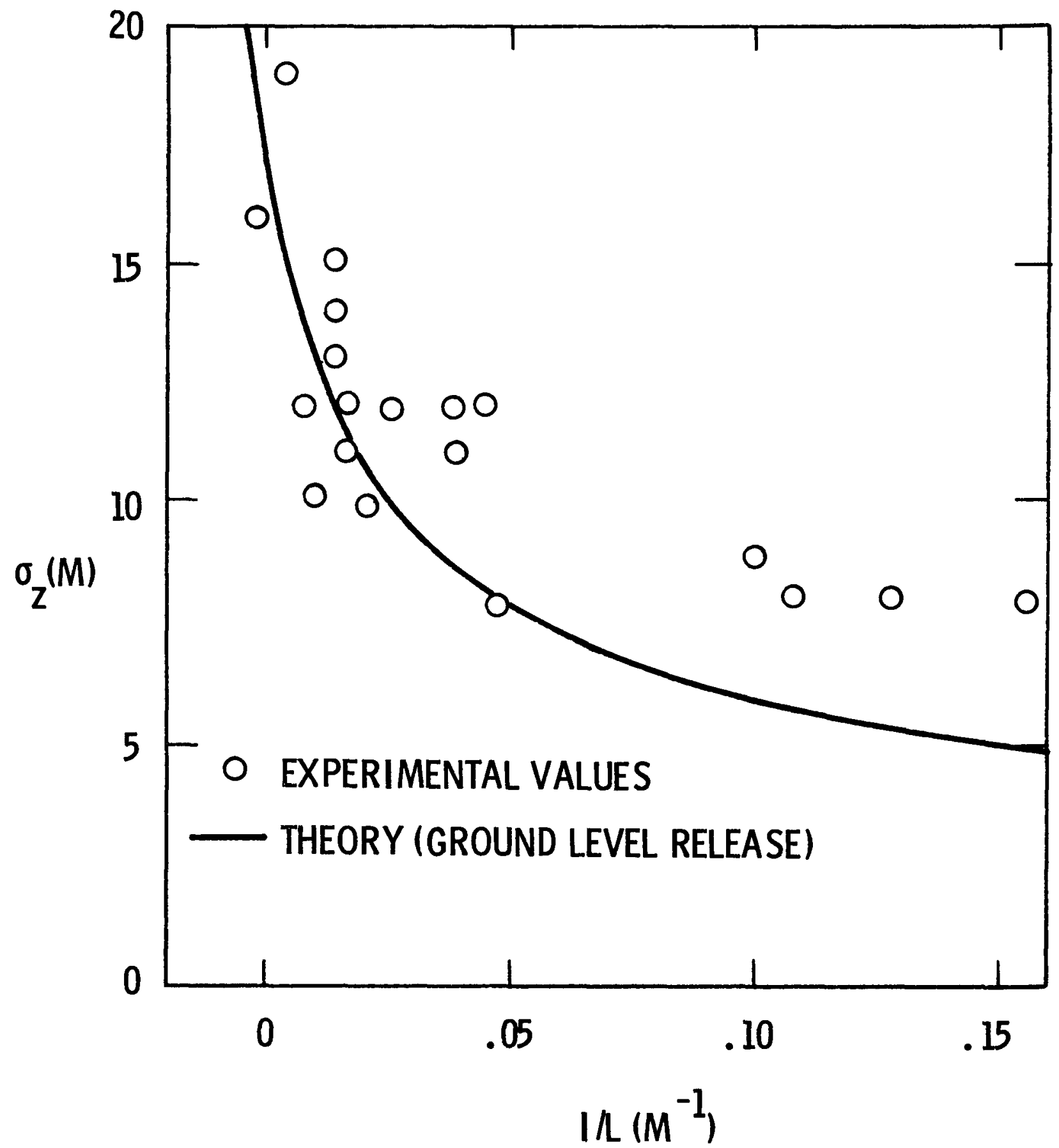

Figure 5.19. Comparison of $\sigma_{7}$ at $400 \mathrm{~m}$ downwind for $26 \mathrm{~m}$ releases with Lagrangian similarity predictions for ground-level releases. 


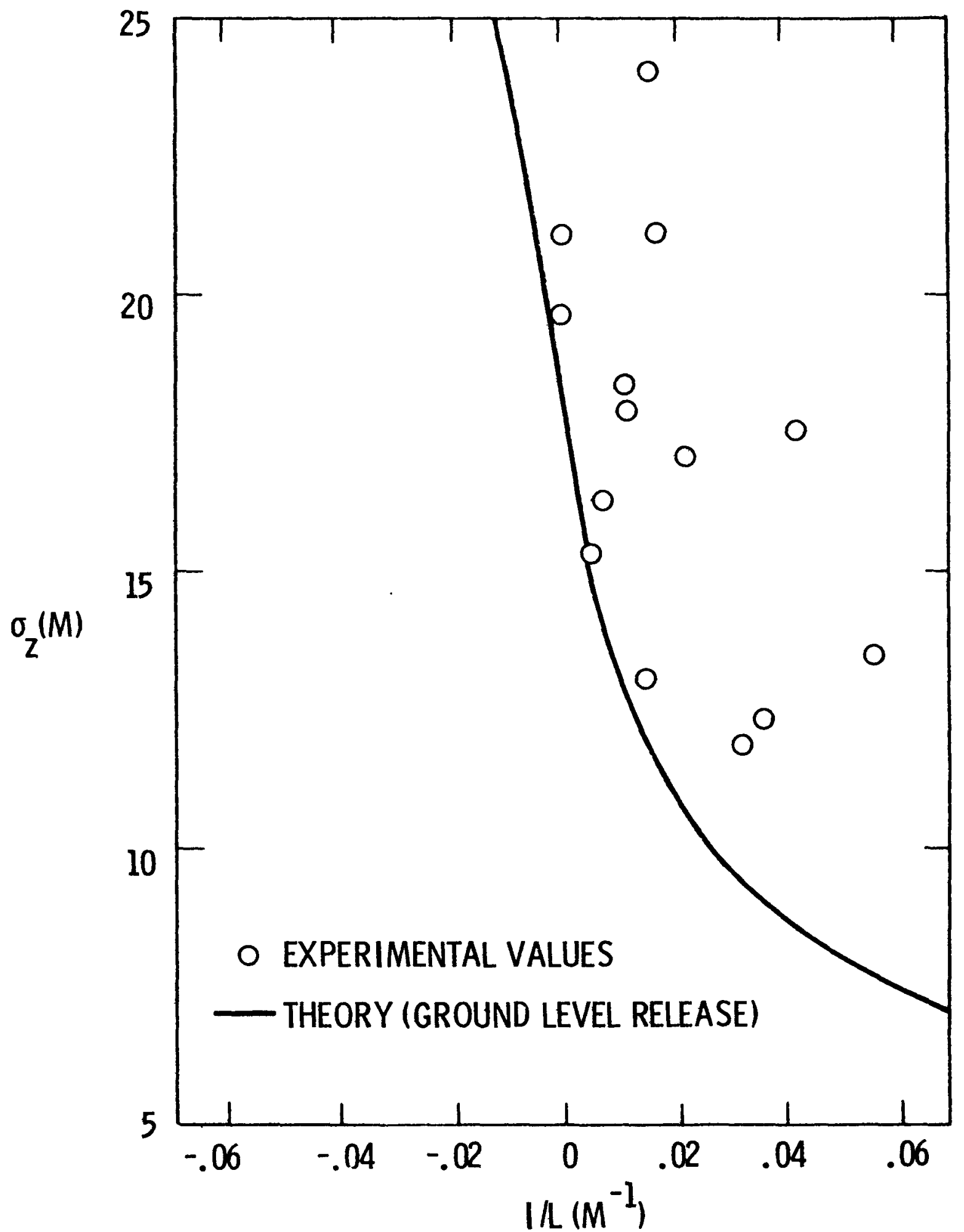

Figure 5.20. Comparison of $\sigma_{z}$ at $400 \mathrm{~m}$ downwind for $56 \mathrm{~m}$ releases with Lagrangian similarity predictions for ground-level releases. 
There were 11 tests in which simultaneous releases were made from both 26 and 56 meters, and for which $\sigma_{z}$ values were obtained from the CWIC at a downwind distance of $400 \mathrm{~m}$. The $\sigma_{z}$ values for the higher release height averaged $1.5 \pm 0.3$ larger than those found for the lower elevation releases. No clearly defined trends with stability were discernible in these comparisons. The results for individual tests are given in Table 5.3 .

Table 5.3. $\sigma_{z}$ at $400 \mathrm{~m}$ from Crosswind-Integrated Concentrations

\begin{tabular}{|c|c|c|c|}
\hline \multirow[b]{2}{*}{ Test } & \multirow[b]{2}{*}{$1 / \mathrm{L}\left(\mathrm{m}^{-1}\right)$} & \multicolumn{2}{|c|}{$\sigma_{z}(m)$} \\
\hline & & $\mathrm{h}=26 \mathrm{~m}$ & $\underline{h}=56 \mathrm{~m}$ \\
\hline U58 & .017 & 11 & \\
\hline U63 & .015 & 12 & \\
\hline U64 & .107 & 8 & \\
\hline U65 & .011 & 10 & \\
\hline U68 & .026 & 12 & \\
\hline U69 & .014 & 15 & \\
\hline U70 & .003 & 19 & \\
\hline $077^{*}$ & .045 & 12 & 21 \\
\hline U72* & .038 & 12 & 17 \\
\hline $073^{*}$ & .014 & 13 & 18 \\
\hline U74* & .048 & 8 & 12 \\
\hline U75* & .128 & 8 & 18 \\
\hline U76* & .008 & 12 & 15 \\
\hline $\mathrm{U} 77^{\star}$ & .020 & 10 & 18 \\
\hline U78* & -.002 & 16 & 21 \\
\hline U79* & .039 & 11 & 12 \\
\hline U81* & .015 & 14 & 24 \\
\hline U82* & .156 & 8 & \\
\hline U83* & .100 & 9 & 13 \\
\hline U85 & -.012 & & 20 \\
\hline U9? & .107 & & 16 \\
\hline U92 & .153 & & 13 \\
\hline
\end{tabular}

* Simultaneous releases from 56 and 26 meters.

There were also a number of cases in which releases were made simultaneously at $26 \mathrm{~m}$ and $2 \mathrm{~m}$. Unfortunately, no tower data were available in these instances. Attempts to evaluate $\sigma_{z}$ from the CWIC of the ground-level releases were not successful, presumably because of significant depletion 
of the plume as it travels downwind. As a result, it is only possible to make the qualitative observations about the relative behavior of ground and elevated releases summarized in Fig. 5.18, 5.19, and 5.20.

\subsection{ELEVATED SOURCES: OTHER DATA}

There have been several additional sets of field experiments designed to study vertical dispersion from elevated sources, but their results are not readily comparable to those obtained in the Hanford 67-Series.

At the National Reactor Test Center in Idaho, 16 releases were made from the top of a $150 \mathrm{ft}$ tower. The analysis of the results, which included only ground level measurements, was made in terms of a Gaussian model. The maximum ground level concentrations were found during "temperature-lapse conditions", and from these a relationship between $\sigma_{z}$ and $\sigma_{\phi}$ was derived, $\sigma_{z}=\sigma_{\phi} x / 1.23 . \quad \sigma_{\phi}$ is the standard deviation of the vertical wind direction. For 82 measurements of concentration along the plume axis, this relationship then resulted in calculated values which agreed with measured values, within a factor of two, 75 per cent of the time.

Other tests were carried out at the Nuclear Research Centers of Karlsruhe and Jülich. Tracer releases were made from heights of 50 and $100 \mathrm{~m}$, over considerably rougher terrain than that found at either Hanford or NRTS. The data collection grid at Karlsruhe was sparser than that at Jülich, but the analysis procedures were similar. Subsequent remarks apply to the Jülich tests, but are generally applicable to the Karlsruhe ones as well.

The most extensive analyses were made in terms of a simultaneous non-linear least squares fit of the data to obtain $\sigma_{y}$ and $\sigma_{z}$ values in a Gaussian formulation. $\sigma_{y}$ and $\sigma_{z}$ were assumed to vary as some power of the downwind distance; exponents and coefficients were found for stability classes A through $F$.

Early tests at Jülich showed that 82 percent of the measurements deviated from the resultant calculated values by less than a factor of three (Vogt et a1, 1974). The investigators also compared their results 
with those predicted on the basis of values obtained from the usual Pasquill curves for various stability categories. They noted that the ground level maxima in their data showed a strong shift toward the source. In addition, maximum concentrations were higher than those predicted from the Pasquill values.

There is a fundamental difficulty in comparing the results of analyses such as these with those based on the Hanford 67 results. The NRTS, Jülich and Karlsruhe approaches are inextricably connected with the framework of a particular model, i. e., the Gaussian formulation of the diffusion process. As has been seen, however, there are serious limitations to this approach. Those results, then, must be regarded as strictly empirical fits to the data, and it is difficult to draw general conclusions from them. Since they are empirical fits, however, they are valid descriptions of the observations and may therefore be useful in predicting ground level concentrations under similar conditions, although a quantitative description of "similar conditions" is not readily available.

In recent papers, Vogt (1977 a,b) has summarized the principal findings of the Jüich studies, and presented them in a series of tables and graphs. He concludes that for extremely unstable conditions, (A stability) diffusion parameters $\left(\sigma_{y}\right.$ and $\sigma_{z}$ ) are larger for $100 \mathrm{~m}$ release heights than for $50 \mathrm{~m}$ release heights. For other stability categories, the opposite is true, with the greatest differences observed for classes $C$ and $D$. He attributes this predominantly to the relatively large surface roughness in the area; $z_{0} \sim 0.4 \mathrm{~m}$ for the area in general and $\sim 1.8 \mathrm{~m}$ near the release point. He also suggests that the Jülich parameters "should be applicable to the majority of potential sites with medium to high surface roughness."

To compare these data with other field programs, Vogt expresses $\sigma_{y}$ and $\sigma_{z}$ as power laws of the form

$$
\text { and } \begin{aligned}
\sigma_{y} & =p_{y} x^{q_{y}} \\
\sigma_{z} & =p_{z} x^{q_{z}}
\end{aligned}
$$


Results were tabulated comparing releases at Brookhaven from a height of $108 \mathrm{~m}$, at St. Louis (ground-leve1), and the Jülich tests. In addition, fits to Pasquill curves in terms of similar power laws were also made. Table 5.4 is extracted from the values given by Vogt (1977b).

Table 5.4. Coefficients and Exponents of Power Laws for $\sigma_{y}$ and $\sigma_{z}$. $\underline{\text { Series }}$ Stability

\begin{tabular}{|c|c|c|c|c|c|c|}
\hline Jutich $(50 \mathrm{~m})$ & $\begin{array}{l}p_{y} \\
q_{y} \\
p_{z} \\
q_{z}\end{array}$ & $\begin{array}{c}\frac{A}{0.869} \\
0.810 \\
0.222 \\
0.968\end{array}$ & $\begin{array}{l}\frac{B}{0.869} \\
0.810 \\
0.222 \\
0.968\end{array}$ & $\begin{array}{c}c \\
0.718 \\
0.784 \\
0.215 \\
0.944\end{array}$ & $\begin{array}{l}\frac{D}{0.625} \\
0.767 \\
0.205 \\
0.936\end{array}$ & $\begin{array}{l}\frac{E}{1.691} \\
0.621 \\
0.162 \\
0.810\end{array}$ \\
\hline $\begin{array}{l}\text { Julich } \\
(100 \mathrm{~m})\end{array}$ & $\begin{array}{l}p_{y} \\
q_{y} \\
p_{z} \\
q_{z}\end{array}$ & $\begin{array}{l}0.229 \\
1.003 \\
0.097 \\
1.158\end{array}$ & $\begin{array}{l}0.227 \\
0.970 \\
0.155 \\
1.024\end{array}$ & $\begin{array}{l}0.224 \\
0.938 \\
0.247 \\
0.890\end{array}$ & $\begin{array}{l}0.222 \\
0.905 \\
0.398 \\
0.755\end{array}$ & $\begin{array}{l}1.691 \\
0.621 \\
0.162 \\
0.809\end{array}$ \\
\hline $\begin{array}{c}\text { Brookhaven } \\
(108 \mathrm{~m})\end{array}$ & $\begin{array}{l}p_{y} \\
q_{y} \\
p_{z} \\
a_{z}\end{array}$ & & $\begin{array}{c}\mathrm{B}_{2} \\
0.400 \\
0.910 \\
0.471 \\
0.907\end{array}$ & $\begin{array}{c}\mathrm{B}_{1} \\
0.360 \\
0.860 \\
0.326 \\
0.859\end{array}$ & $\begin{array}{c}c \\
0.320 \\
0.780 \\
0.223 \\
0.776\end{array}$ & $\begin{array}{c}D \\
0 . \overline{310} \\
0.710 \\
0.062 \\
0.709\end{array}$ \\
\hline $\begin{array}{c}\text { St. Louis } \\
\text { (ground } \\
\text { level) }\end{array}$ & $\begin{array}{l}p_{y} \\
q_{y} \\
p_{z} \\
q_{z}\end{array}$ & & $\begin{array}{l}\frac{B}{1.700} \\
0.717 \\
0.079 \\
1.200\end{array}$ & $\begin{array}{l}\frac{c}{1.440} \\
0.710 \\
0.131 \\
1.046\end{array}$ & $\begin{array}{c}D \\
0.910 \\
0.729 \\
0.910 \\
0.702\end{array}$ & $\begin{array}{l}\frac{E}{1.020} \\
0.648 \\
1.930 \\
0.465\end{array}$ \\
\hline
\end{tabular}

The observation that $\sigma_{z}$ is larger for $50 \mathrm{~m}$ releases than for $100 \mathrm{~m}$ releases in neutral and stable conditions does not agree with the measured behavior of that quantity for 26 and $56 \mathrm{~m}$ releases at Hanford. Whether this is due to the different roughness characteristics is not certain, al though this feature might well be expected to influence the behavior of diffusing plumes.

In the section on the analysis of $\sigma_{y}$, it was also shown from the Hanford data that $\sigma_{y} / x \sigma_{\theta}$ tends to become independent of release height at 
sufficiently large downwind distances and for $\sigma_{\theta}$ measured at ground level. This is also contrary to the Jülich results, where $\sigma_{y}$ (and hence $\sigma_{y} / \mathrm{x}_{\theta}$ ) depends on release height. Once again, terrain roughness is a likely cause for this effect, but as yet it is not possible to quantify its influence.

\subsection{CONCLUSIONS}

Lagrangian similarity theory provides a good description of the vertical dispersion from ground-level sources to a dimensionless distance of at least $x / z_{0}=2 \cdot 10^{5}$. Observed CWIC clearly show that the eddy diffusivity of a passive contaminant corresponds more closely to that of heat than that of momentum, and they also support the assumption that the effect of atmospheric stability on the vertical distribution is to alter the scale of the diffusion without strongly affecting the form of the distribution. The standard Gaussian formula (5.16) is recommended for predicting the CWIC from ground-level sources, with $\sigma_{z}$ computed from Eq. 5.5 and $u$ specified at the height $0.5 \sigma_{z}$.

Al though the data are still relatively sparse, some general observations can be made about vertical dispersion characteristics from elevated sources.

For 26 meter releases, the initial profile is fairly well described by the Gaussian form for diffusion in the presence of a perfectly reflecting plane. This is presumably true for more elevated releases as well.

As $\sigma_{z}$ of this Gaussian increases to become comparable with the release height $h$, the Gaussian provides an increasingly poor description of the plume shape. Examination of results from tests $V 1$ through $V 8$ suggest that simple corrections for deposition effects are probably not enough to account for the observed deviations.

The use of the CWIC at ground level provides a useful means of estimating $\sigma_{z}$ provided $\sigma_{z} \lesssim 0.7 \mathrm{~h}$.

For stable conditions, Lagrangian similarity theory for ground-level sources may significantly underestimate the vertical dispersion for an elevated source. For less stable conditions, these predictions appear to 
provide an adequate description for elevated sources as wel1. However, as the height of the source increases, the Monin-Obukhov length scale may no longer be a useful parameter for stability classification, at least under stable stratification.

Under neutral to moderately stable conditions, at $400 \mathrm{~m}$ downwind from the source, $\sigma_{z}$ for $56 \mathrm{~m}$ releases is approximately 1.5 times larger than $\sigma_{z}$ for 26 meter releases, at least in relatively smooth terrain.

The Jülich results provide a valuable addition to the description of the characteristics of plumes from elevated releases, provided they are used only for predictions of ground level concentrations. To date, it is not possible to specify how roughness length may be incorporated specifically into elevated-release calculations. In areas of moderately large roughness elements, however, the parameters derived from the Jülich data may provide a substantial improvement over previous estimates of $\sigma_{z}$ appropriate for use in a Gaussian formula.

A possible difficulty may arise in applying Jülich results if $\sigma_{y}$ is determined or estimated independently of $\sigma_{z}$. In that case, the leastsquares values of $\sigma_{z}$ determined for a particular stability class may not provide an accurate concentration estimate unless the actual $\sigma_{y}$ value is also similar to that determined for the same stability class. Such a situation might arise, for example, if $\sigma_{y}$ were independently estimated from $\sigma_{\theta}$. 
6. THE NET EFFECT OF INCREASED SOURCE HEIGHT ON GROUND-LEVEL CONCENTRATIONS

\section{I INTRODUCTION}

There is no doubt that the ground level concentration of a poliutant at near-source distances can be reduced by increasing the height at which the pollutant is released to the atmosphere. However, from a practical standpoint the benefits in lower concentration must be balanced against the increased cost incurred in constructing taller stacks. How tall is tall enough? Modeling can give some guidance. In this chapter, guidance based on measurements is offered. The benefits accruing by virtue of increasing release height over several specific increments are examined.

Experimental investigation of source height effects has conventionaliy entailed the release of a tracer or pollutant from one elevation under certain meteorological conditions, and then repeating the release from a different elevation at a time when the meteorology is similar to that existing during the first release. Unfortunately, the free atmosphere is an uncooperative laboratory which never precisely repeats itself, and thus the effects of such a variable as source height can be somewhat obscured by the changes in meteorology between experiments. Further, there remains the problem that "similar" meteorology is relative to our understanding or modeling of atmospheric diffusion. In other words, even if such parameters as vertical temperature gradient, wind speed gradient, and wind direction variances are found to be identical on two separate occasions, the observed concentrations of a tracer might well vary significantly because more than the measured parameters are affecting the diffusion. The simultaneous release from more than one elevation of atmospheric tracers with similar aerodynamic properties reduces this problem.

\subsection{EXPERIMENTAL APPROACH}

In order to employ a dual tracer technique in source height investigations, it is necessary to establish the compatability of two tracers. 
Simultaneous releases of the tracers zinc sulfide FP 2210* and fluorescein ${ }^{\star \star}$ began at the Hanford Reservation in 1964. This dual tracer technique (Nickola, 1965; Ludwick, 1966) involves simultaneous collection of the tracers on a common set of filters, and subsequent noninterfering assays for each tracer. The compatibility of the tracers was investigated in the field in several experiments in which the two tracers were released from the same location. The Hanford 67-Series data volume (Nickola, 1977) documents four of these common-source experiments and 34 dual-releaseheight experiments. The dispersal technique employed in the common-source experiments (Tests D1, D2, D3 and D4) was identical to that used in the dual-level releases.

More than 400 filter samplers were exposed during each of the commonrelease-point experiments. More than half the samplers were deployed on an array of 20 towers, while the remainder were deployed at an elevation of $1.5 \mathrm{~m}$. Sampling was done on arcs at radial distances of 200,800, 1600 and $3200 \mathrm{~m}$ from the source. The release point was at an elevation of $2 \mathrm{~m}$.

Inasmuch as identical masses of fluorescein ( $F L)$ and zinc sulfide ( $\mathrm{ZnS}$ ) were released, identical masses collected on filters in the common-releasepoint experiments would have indicated perfect compatibility of tracers. Such was not the case. Fig. 6.1 shows the ratio of FL to $\mathrm{ZnS}$ observed at each sampling arc. Twelve $\mathrm{FL} / \mathrm{ZnS}$ ratios ${ }^{\star \star *}$ contribute to each plotted logarithmic mean. Error bars depicting $\pm 1 \sigma$ about the mean value are included on the figure. Ratios of FL to $\mathrm{ZnS}$ observed in the 31 usable dual-release-height experiments were adjusted on the basis of the curve presented, so that any difference in observed concentration was presumed to be due primarily to release height and not to tracer properties.

* A fluorescent pigment manufactured by the U.S. Radium Corp., Morristown, N.J.

** A water soluble uranine dye manufactured by (among others) Allied Chemical Co., San Francisco, California.

*** For each of four experiments, these are the ratios of the peak concentrations at ground level $(1.5 \mathrm{~m})$, the ground-level crosswind-integrated concentrations, and the peak concentrations observed on a tower. 


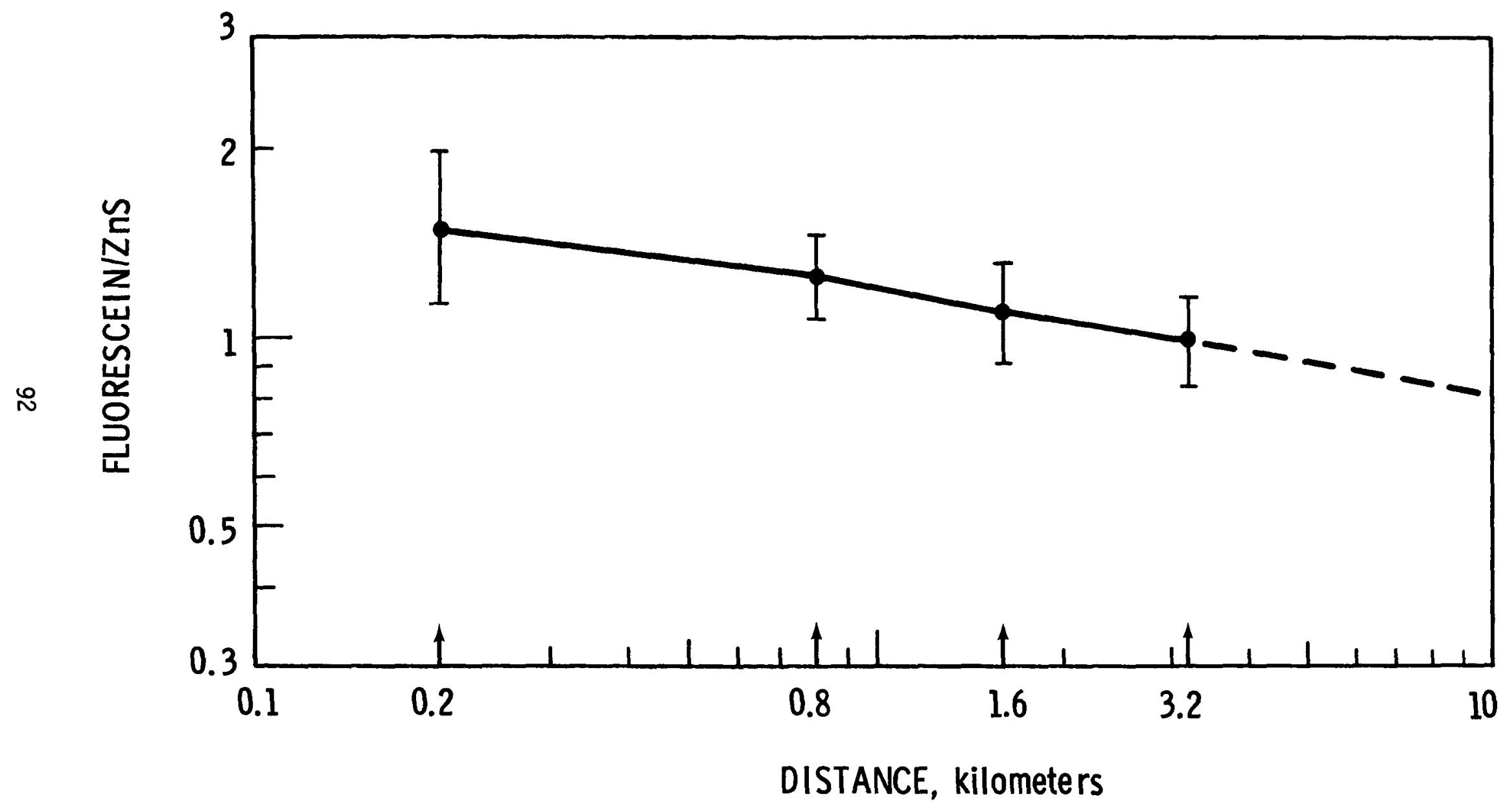

Figure 6.1. The observed ratio of fluorescein to zinc sulfide tracers following release from a common source. 
It was concluded that investigation of source height effects could best be done through crosswind-integrated concentration rather than plume centerline concentration, although ratios formed from either measurement were generally of similar magnitude. Since CWIC at a specific distance from a source is based on a number of measurements (as opposed to a single measurement for the centerline exposure), the CWIC is a more reliable parameter. Furthermore, it is the CWIC summed over a meteorologically long period of time that prescribes the dosage available to receptors at any distance.

The field releases, generally 30 minutes in duration, were divided into Pasquil1-Gifford stability categories on the basis of temperature differences as specified in U. S. NRC Regulatory Guide 1.23 (USNRC, 1972). This classification prescribes measurement of a temperature differential between $40 \mathrm{~m}$ and $10 \mathrm{~m}$ for releases at or below $40 \mathrm{~m}$. For releases above $40 \mathrm{~m}$, the differential between the release height and $10 \mathrm{~m}$ is used. Ordering of the experiments on the basis of bulk Richardson number showed minimal variation from the $\Delta T / \Delta z$ ordering.

Fig. 6.2 gives CWIC values observed at ground level during field experiment U78, an experiment carried out during class D stability. The dots represent CWIC's resuiting from $\mathrm{ZnS}$ released at an elevation of $26 \mathrm{~m}$. The solid squares depict CWIC's resulting from a FL release from $56 \mathrm{~m}$. Heavy smooth curves are sketched through the data points. The data have been normalized to mass rate of tracer emission, but not to wind speed since the intent is to present difference in exposures actually observed with equal releases. The light curves on Fig. 6.2 are CWIC's for class $D$ stability resulting from a Gaussian plume model and the specific wind speeds observed at each release height. The Gaussian equation prescribes that at ground level,

$$
\text { CWIC }=\frac{2}{\sqrt{2 \pi} \sigma_{z} \bar{u}_{h}} \exp \left(-\frac{h^{2}}{2 \sigma_{z}^{2}}\right)
$$

where $\sigma_{z}$ is the standard deviation of the plume vertical concentration 


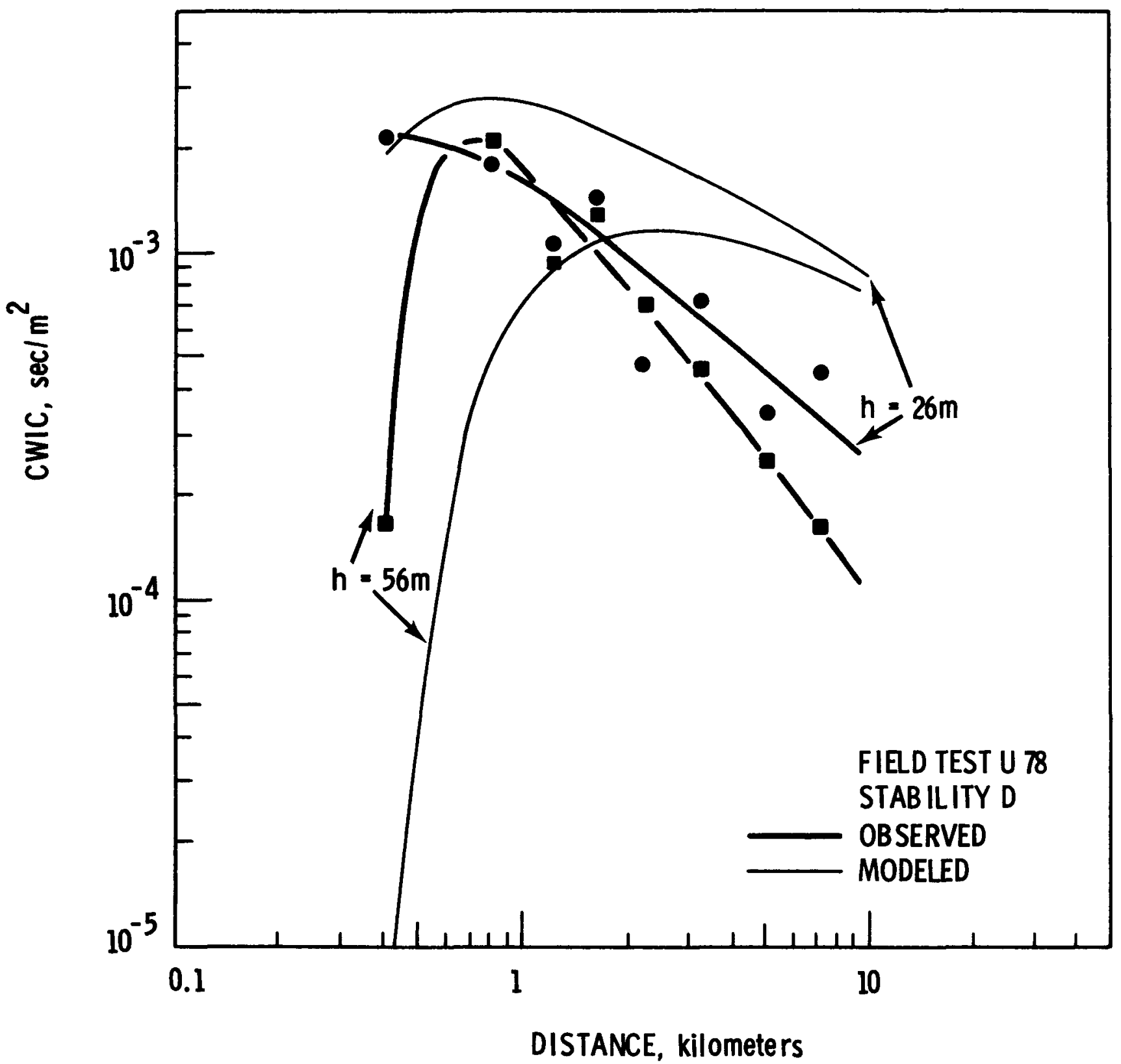

Figure 6.2. Normalized CWIC values observed during field test U78. $h$ indicates tracer release height. 
distribution, $\bar{u}_{h}$ is the mean wind speed at source height and $h$ is the source height. In this form, total plume reflection without deposition is assumed. Turner Workbook values of $\sigma_{z}$ were used. The use of the Gaussian model with stability based on $\Delta T / \Delta z$ is not intended as an endorsement or a condemnation, but merely as a plane of reference.

Figure 6.3 presents data similar to Figure 6.2, but for field test U79 which was completed during class F stability. Note that both the observed and the modeled curves reflect the less rapid diffusion of tracer to the surface during the more stable test U79. However, the modeled and observed curves are certainly not in good agreement in either test U78 or U79.

Ratios, formed by dividing the observed CWIC from the upper level release by the observed CWIC from the lower level release, were computed for selected distances. These ratios were then adjusted in accordance with the curve presented in Fig. 6.1. The summary curves to be presented on Figs. 6.4 to 6.6 are the logarithmic means of the adjusted ratios from the individual experiments.

\subsection{DATA AND DISCUSSION}

The substance of this chapter is presented in Figs. 6.4, 6.5 and 6.6. The bold solid curves in these figures present observed ratios of CWIC resulting from simultaneous tracer releases from two elevations. The diffusion processes leading to these results may be simple or complex, but the curves reflect the result irrespective of the process. They offer guidance based on observation rather than theory and modeling.

Table 6.1 lists the 67-Series field experiments (with pertinent wind speed and thermal stability data) used in the source height effects investigation. Three combinations of upper/lower release elevations were used: $26 \mathrm{~m} / 2 \mathrm{~m}, 56 \mathrm{~m} / 26 \mathrm{~m}$, and $111 \mathrm{~m} / 56 \mathrm{~m}$. In the 24 releases involved in the $26 \mathrm{~m} / 2 \mathrm{~m}$ and $56 \mathrm{~m} / 26 \mathrm{~m}$ pairings, stability classification based on NRC Regulatory Guide 1.23 resulted in the same stabilities for upper and lower releases. However, examination of stabilities in Table 6.1 for the $111 \mathrm{~m} / 56 \mathrm{~m}$ 


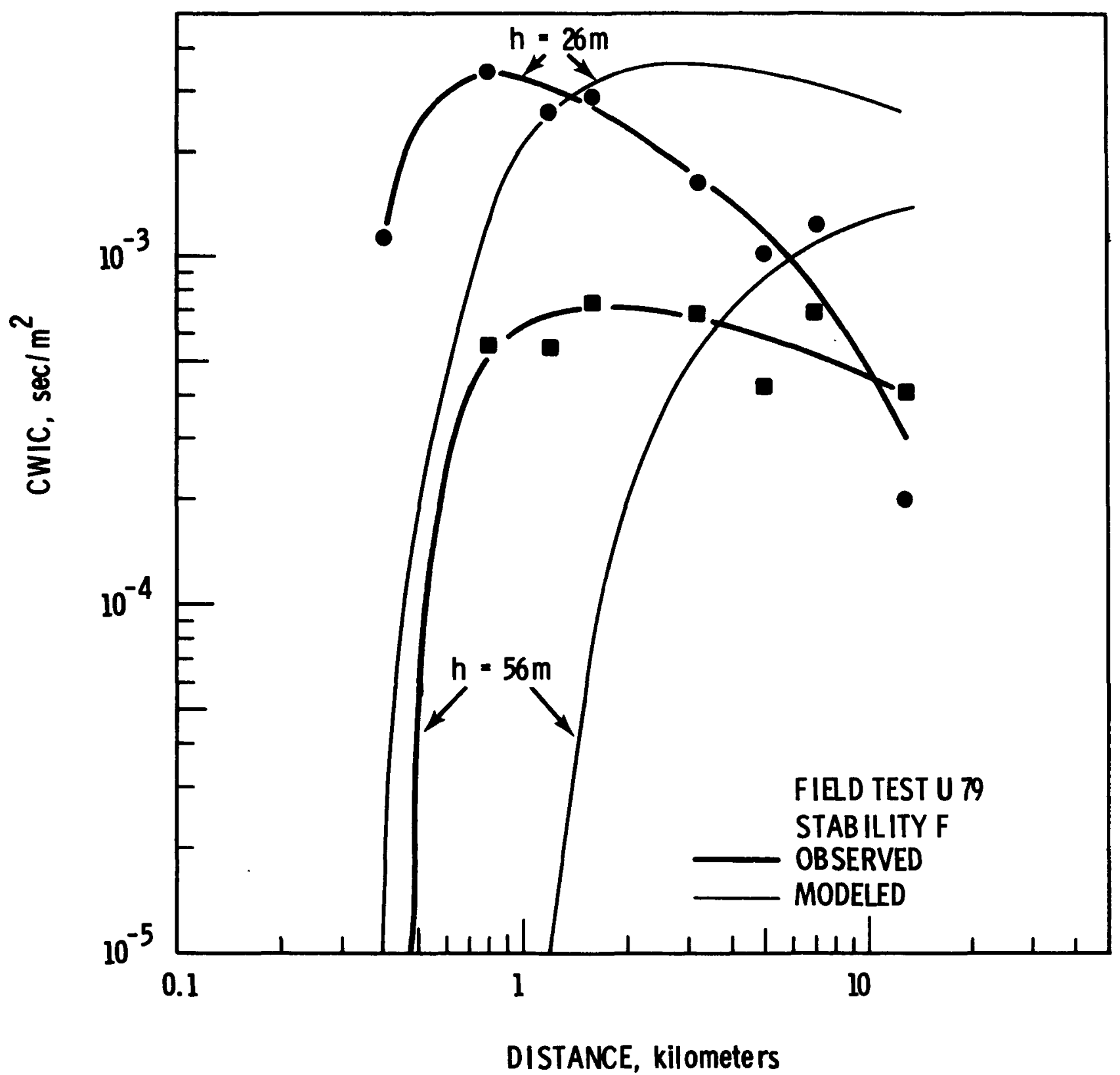

Figure 6.3. Normalized CWIC values observed during field test U79. $h$ indicates tracer release height. 
Table 6.1. Source Height, Wind Speed and Stability for Dual Releases.

\begin{tabular}{|c|c|c|c|c|c|c|c|c|}
\hline $\begin{array}{l}\text { IEST } \\
\text { NOE. }\end{array}$ & $\dashv \mathrm{T}$. & $\begin{array}{l}\text { WIVU } \\
\text { SPEED }\end{array}$ & $\frac{\partial E L(T)^{\ddagger}}{D E L(Z)}$ & STAB. & HT. & $\begin{array}{l}\text { WI YD } \\
\text { SPEED }\end{array}$ & $\frac{D E L(T)}{D E L(Z)} \neq$ & STAB. \\
\hline--- & --- & $M / S E C$ & $\mathrm{c} / 100 \mathrm{M}$ & ---- & $M$ & M/SEC & $\mathrm{C} / 100 \mathrm{M}$ & - \\
\hline Jót & 26 & $5 \cdot 3$ & 9.9 & G & 2 & 1.5 & 9.9 & $\ddot{0}$ \\
\hline 457 & 26 & 4.3 & 3.4 & 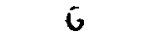 & 2 & 1.5 & 4.3 & $G$ \\
\hline 460 & 26 & 4.0 & 4.2 & 0 & 2 & 1.2 & 4.2 & 6 \\
\hline J 56 & 26 & 3.5 & 3.9 & $F$ & 2 & 1.4 & 3.9 & $f$ \\
\hline 156 & 26 & 4.3 & $\Xi .7$ & $F$ & 2 & 1.0 & 3.7 & $f$ \\
\hline $46 \approx$ & $? 5$ & 4.7 & 1.5 & $E$ & 2 & 2.4 & 1.5 & $E$ \\
\hline 465 & 26 & 5.5 & 1.5 & $E$ & 2 & 3.3 & 1.5 & $E$ \\
\hline 153 & 26 & 5.0 & 1.2 & E & 2 & 3.0 & 1.2 & E \\
\hline U5y & 26 & 3.4 & 1.1 & $E$ & 2 & $1 . y$ & 1.1 & $\vec{E}$ \\
\hline 161 & 26 & 8.5 & C. 2 & $E$ & 2 & 5.2 & 0.2 & $E$ \\
\hline 470 & 26 & 7.0 & $\therefore .0$ & $t$ & 2 & 4.0 & 0.0 & $\vec{E}$ \\
\hline U6? & 25 & 3.4 & -3.7 & $\bar{v}$ & 2 & 5.4 & -0.7 & U \\
\hline is 3 & 56 & 5.6 & $5 . c$ & $G$ & 25 & 5.2 & 8.4 & v \\
\hline U80 & 56 & 2.8 & 6.4 & C & 26 & 2.2 & 7.6 & $G$ \\
\hline 1182 & 56 & 4.6 & 5.6 & 6 & 26 & 4.7 & 7.6 & u \\
\hline 479 & 53 & 3.5 & 3.1 & $F$ & 25 & 5.1 & 3.5 & $F$ \\
\hline 074 & 56 & 6.2 & 2.3 & $F$ & 25 & 4.5 & 2.6 & $F$ \\
\hline U72 & 55 & $5 \ldots$ & 2.3 & $F$ & 26 & 4.4 & 2.4 & $F$ \\
\hline U\$1 & 56 & 7.0 & 1.5 & $F$ & 26 & 5.7 & 1.9 & $F$ \\
\hline 076 & 56 & 7.1 & 1.2 & $E$ & 26 & 6.5 & 1.4 & E \\
\hline 473 & 56 & 6.5 & 1.2 & $E$ & 26 & 5.5 & 1.2 & $E$ \\
\hline บ71 & 55 & 4.2 & 3.9 & $E$ & 23 & 3.4 & 1.1 & $k$ \\
\hline 077 & 56 & 3.7 & 0.2 & $\mathrm{E}$ & 25 & 3.3 & 0.4 & $E$ \\
\hline 073 & 55 & $7 \cdot 3$ & -3.54 & D & 26 & 6.5 & -0.7 & $D$ \\
\hline Us 3 & 111 & 6.4 & 2.8 & $F$ & 56 & 7.6 & 5.2 & G \\
\hline U35 & 111 & 5.2 & 1.9 & $F$ & 55 & 4.9 & 4.3 & 6 \\
\hline 492 & 111 & 5.4 & 1.5 & $F$ & 55 & 4.7 & 2.5 & $F$ \\
\hline 490 & 111 & 4.1 & $1.4 d$ & E & 58 & 4.3 & 3.5 & $F$ \\
\hline 189 & 111 & 3.6 & 1.4 & $E$ & 56 & 3.6 & 3.5 & $F$ \\
\hline U91 & $11^{\prime} 1$ & 4.1 & 0.7 & $\hat{\varepsilon}$ & 56 & 3.3 & $2 \cdot 3$ & $F$ \\
\hline Us 5 & 111 & 9.7 & 1.1 & $\hat{E}$ & 56 & 8.2 & 1.1 & E \\
\hline
\end{tabular}

\# For $h=2 m$ release, $\Delta Z=40 \mathrm{~m}-10 \mathrm{~m}$; for $h=26 \mathrm{~m}, \Delta Z=40 \mathrm{~m}-10 \mathrm{~m}$; for $h=56 \mathrm{~m}$, $\Delta Z=56 \mathrm{~m}-10 \mathrm{~m}$; and for $h-111 \mathrm{~m}, \Delta Z=111 \mathrm{~m}-10 \mathrm{~m}$. (USNRC Reg Guide 1.23) 


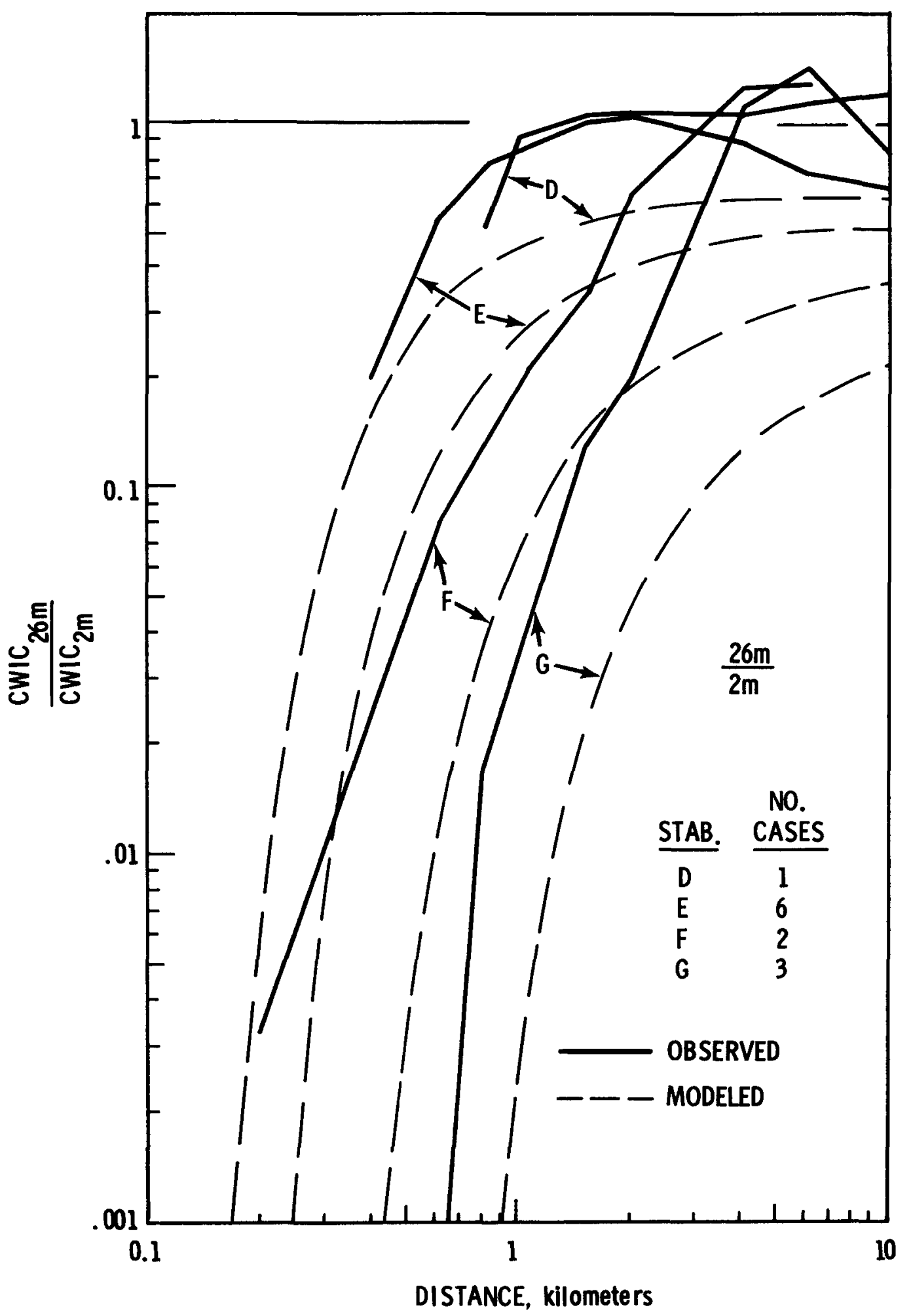

Figure 6.4. Ratio of normalized CWIC observed at $26 \mathrm{~m}$ to that observed at $2 \mathrm{~m}$. Bold curves are observed values; lighter curves are modeled. 


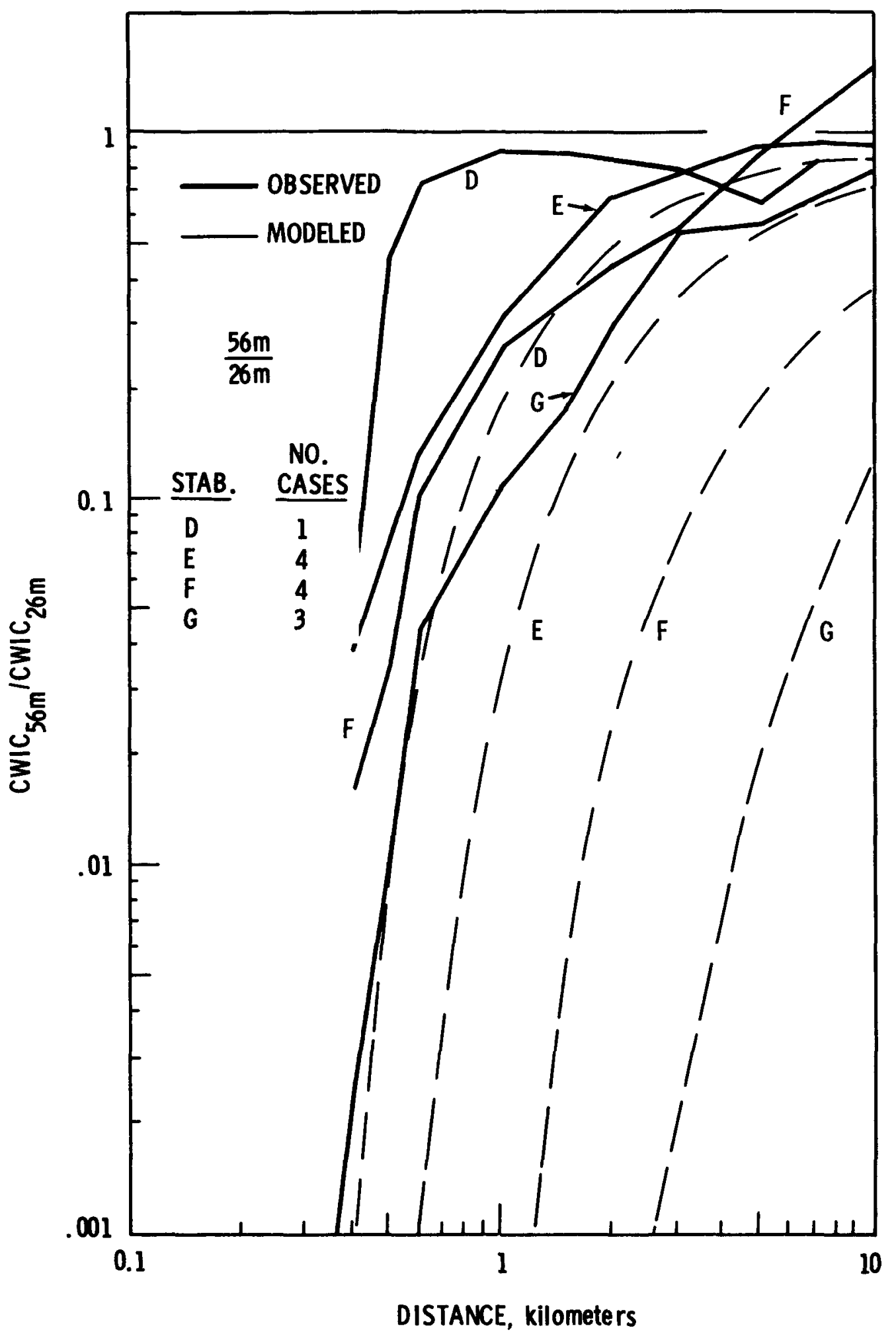

Figure 6.5. Ratio of normalized CWIC observed at $56 \mathrm{~m}$ to that observed at $26 \mathrm{~m}$. Bold curves are observed values; 1ighter curves are modeled. 


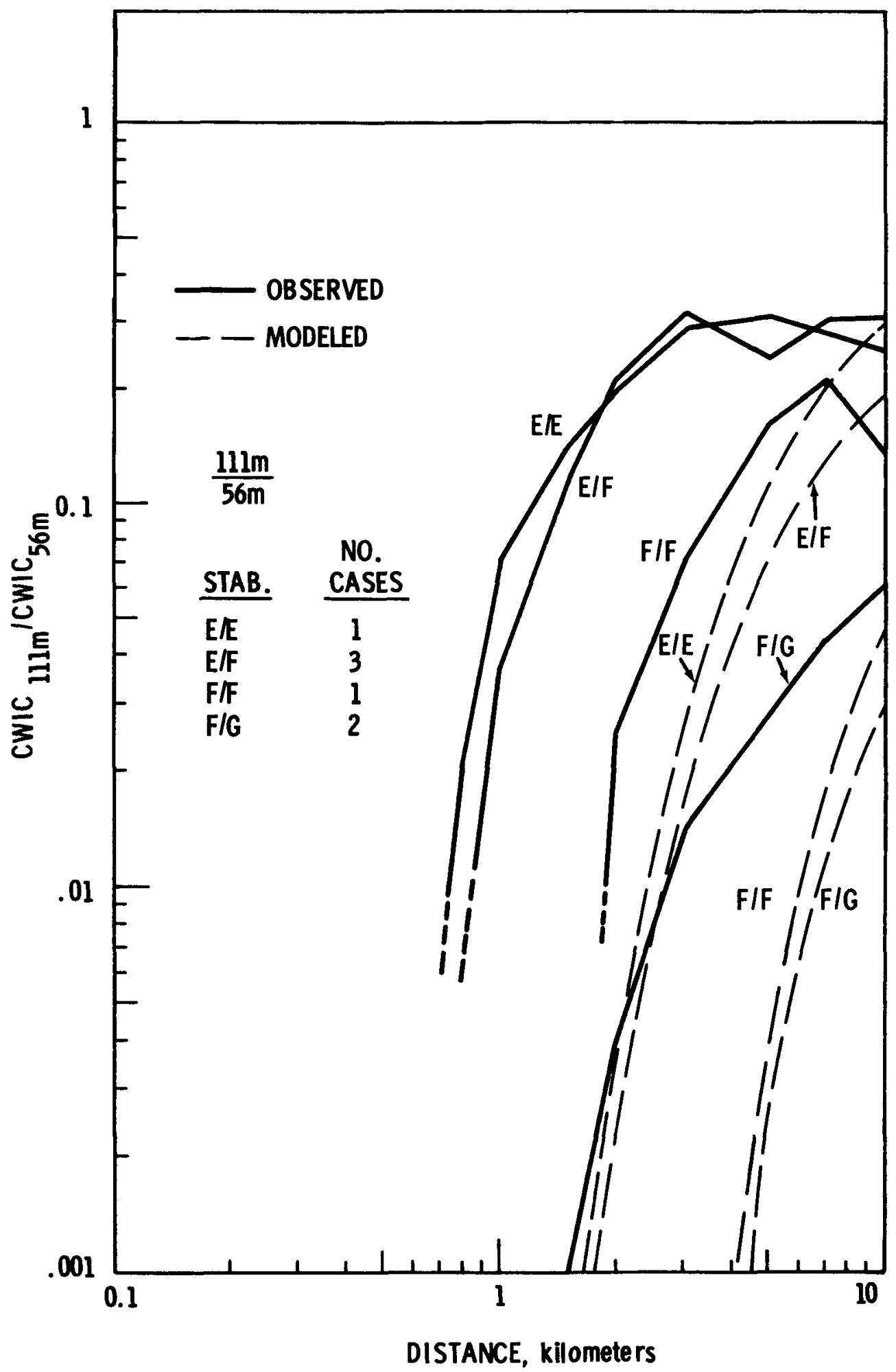

Figure 6.6. Ratio of normalized CWIC observed at $111 \mathrm{~m}$ to that observed at $56 \mathrm{~m}$. Bold curves are observed values; lighter curves are modeled. 
pairings reveals a less stable specification for the upper level release in five of the seven pairings. (This fact necessitated the dual-stability labeling on Fig. 6.6. For instance, the label $F / G$ indicates the $111 \mathrm{~m}$ release was into a class $F$ layer while the $56 \mathrm{~m}$ release was into a class $G$ layer).

The range of stabilities ( $D$ through $G$ ) observed during the 31 field experiments reflects the fact that all the experiments were carried out during night hours. No unstable data are present. Despite this restriction to stable atmospheres, the distribution of the 31 experiments based on 3 pairings of release height with 4 stability classifications for each pairing leaves relatively few experimental cases for input to each of the relationships depicted on Figs. 6.4 to 6.6. (The number of cases input to each curve is indicated on these figures). Yet, the curves stratify reasonably well into the order one might intuitively expect. The benefits increase with increasingly stable stratification.

Before proceeding with a more detailed examination of Figs. 6.4 to 6.6 , it should be pointed out that irrespective of the model used or of the subsequent diffusion process postulated, the initial dilution of a release to the atmosphere is directly proportional to the wind speed at the release point. Thus, if releases are made from two elevations with a wind speed differential, but are presumed to diffuse in identical fashion, the limiting ratio of CWIC's (at distances far removed from the sources) is the inverse of the ratio of the initial wind speeds. At least in a modeled situation, the reduction of ground level CWIC due to an increase in release height can be considered a result of two causes: (1) the increased diffusion between source height and ground level, and (2) the initial dilution due to generally higher wind speeds at higher elevations (to be referred to as the "wind speed benefit").

The dashed curves on Figs. 6.4 to 6.6 are ratios resulting from the Gaussian model. The wind speeds used in developing these curves are the specific speeds observed during the field experiments. Since wind speeds (and hence modeled CWIC ratios) were not identical from experiment to experiment, an averaged modeled ratio is presented. This graphed average is the 
logarithmic mean of the ratios from the contributing experiments. At great distances these curves become asymptotic to the mean ratio of wind speeds observed at the lower and upper elevations--the "wind speed benefit".

Although detailed discussion of Figs. 6.4 to 6.6 is rather superfluous, a few comments are in order. An interpretive example may also prove helpful.

Let us first postulate a release from an elevation of $26 \mathrm{~m}$ into an atmosphere of class $E$ thermal stability. If the release were from $56 \mathrm{~m}$ instead, what would be the reduction of CWIC at $1 \mathrm{~km}$ from the source? The solid curve (observed) labeled $E$ on Fig. $\cdot 6.5$ suggests the $56 \mathrm{~m}$ release height would result in a reduction of the CWIC at $1 \mathrm{~km}$ to about $30 \%$ of that resulting from the $26 \mathrm{~m}$ release. The dashed curve (modeled) implies a value of about $3 \%$-- a much different reduction than the observed data show. In fact the modeled ratios are much smaller (and hence the increased stack benefits are larger) than are the observed ratios on all the paired release heights of Figs. 6.4, 6.5 and 6.6.

The limiting "wind speed benefit," resulting from the observed wind speed differential between the two levels, prescribes ratios that average roughly 0.75 to 0.90 . At greater distances, the observed CWIC ratios from the $56 \mathrm{~m} / 26 \mathrm{~m}$ pairings of Fig. 6.5 generally plateau at about this value. The observed ratios for the $26 \mathrm{~m} / 2 \mathrm{~m}$ pairings of Fig. 6.4 tend to plateau nearer a ratio of unity -- implying an absence of any wind speed benefit. Conversely, the observed $111 \mathrm{~m} / 56 \mathrm{~m}$ pairings of Fig. 6.6 plateau at ratios considerably below 0.75 to $0.90--$ implying a limiting benefit greater than that derived solely from the higher source wind speed.

The de facto pairings of $26 \mathrm{~m} / 2 \mathrm{~m}, 56 \mathrm{~m} / 26 \mathrm{~m}$ and $111 \mathrm{~m} / 56 \mathrm{~m}$ permit the computation of ratios for other pairings when common stability classes are involved. Ratios for these deduced pairings -- based on the observed ratios -- are presented on Figs. 6.7 to 6.9.

The effects of increased source height presented in this chapter are net effects observed for the particulate tracers zinc sulfide and fluorescein over relatively flat terrain. An effort was made to eliminate differences in observed concentrations due to differences in the aerodynamic properties of these specific particulates. Strictly speaking, 


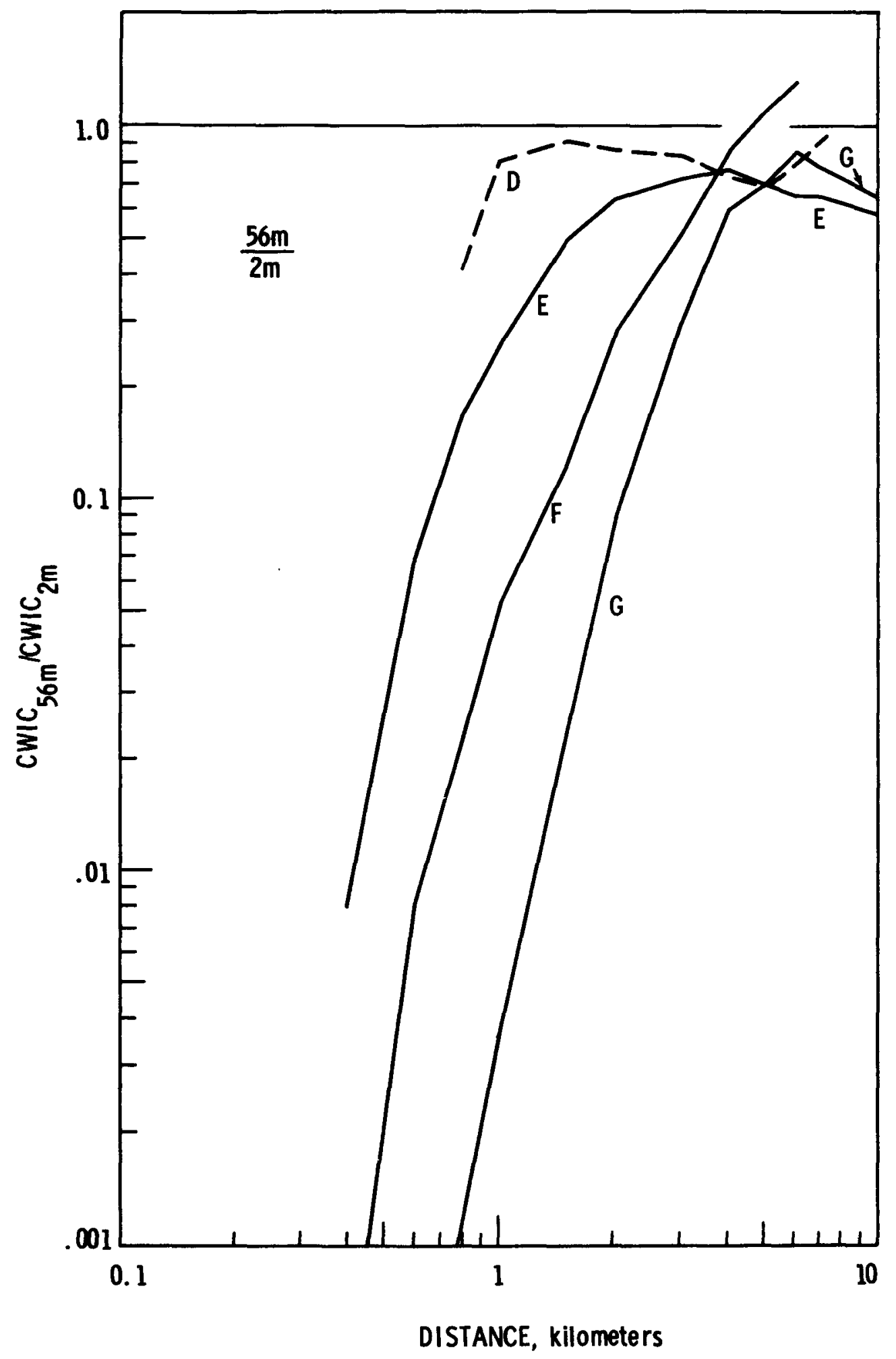

Figure 6.7. Ratio of normalized CWIC at $56 \mathrm{~m}$ to that at $2 \mathrm{~m}$ deduced from observed ratios. 


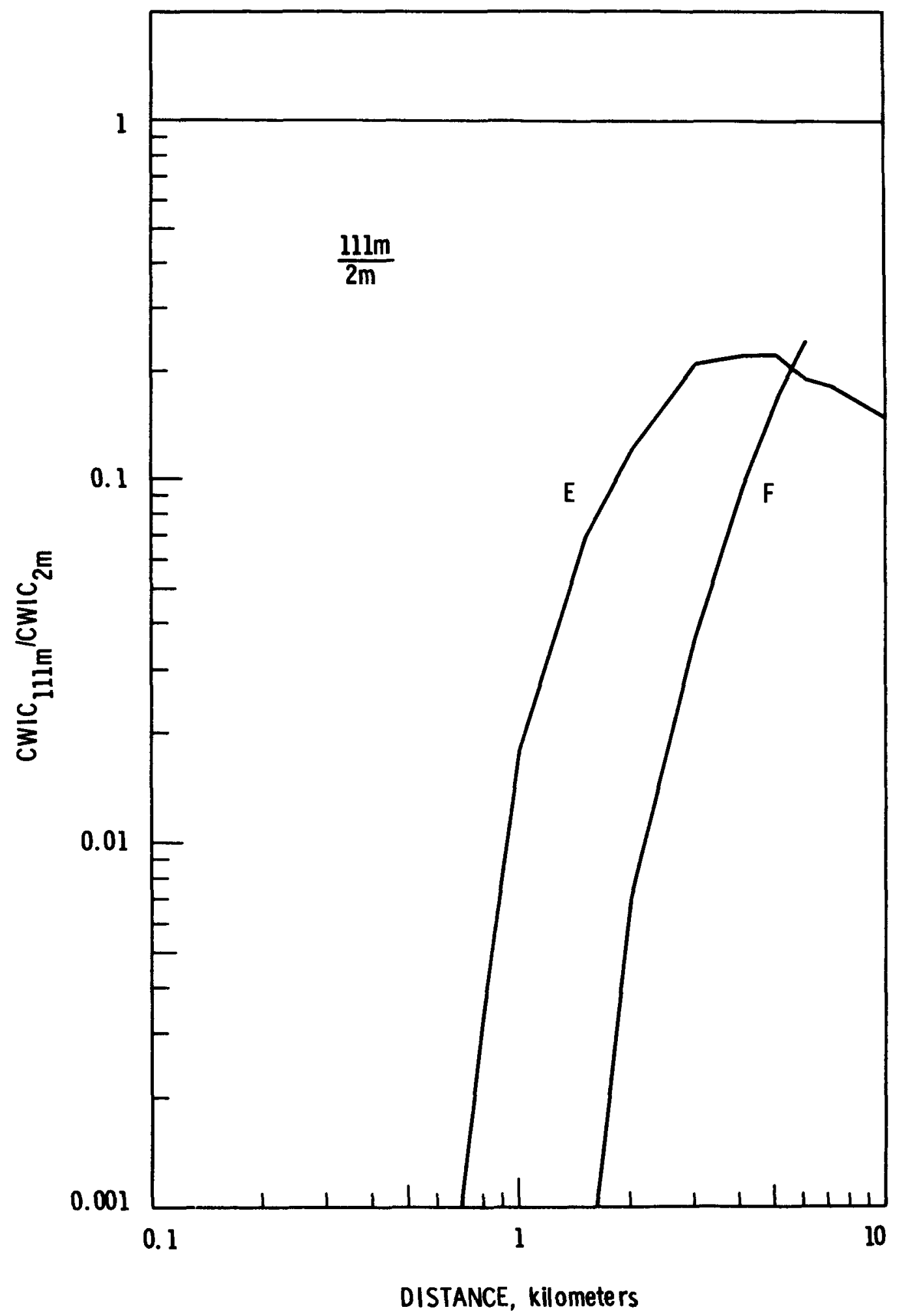

Figure 6.8. Ratio of normalized CWIC at $111 \mathrm{~m}$ to that at $2 \mathrm{~m}$ deduced from observed ratios. 


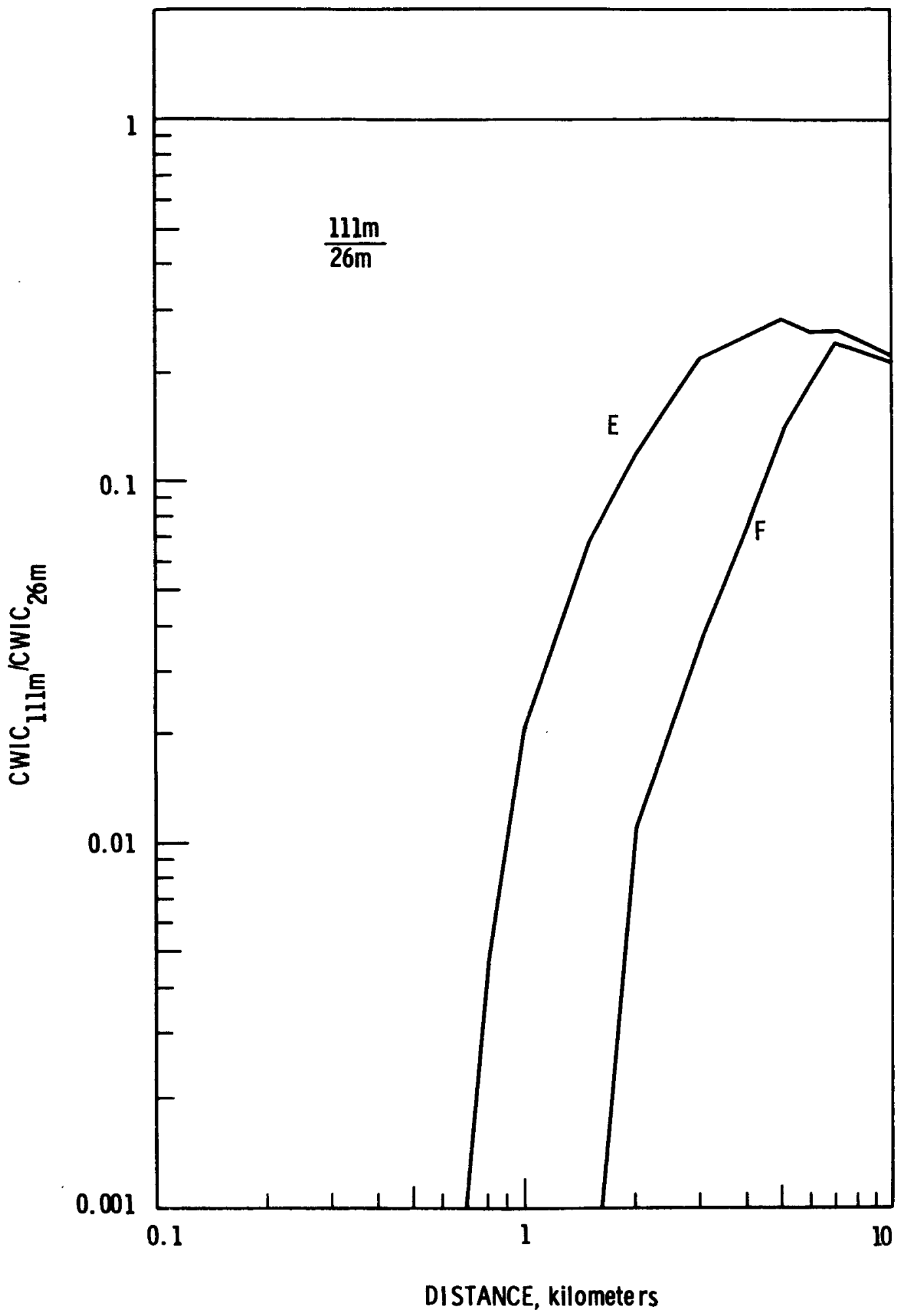

Figure 6.9. Ratio of normalized CWIC at $111 \mathrm{~m}$ to that at $26 \mathrm{~m}$ deduced from observed ratios. 
the ratios apply only to a tracer with properties similar to the two used. For instance, such processes as deposition, gravitational settling or chemical transformations during transport may have uniquely influenced the results presented. However, the tracers employed are relatively small particulates and appear to be chemically stable in the mode they were employed. More detail on the physical characteristics of these tracers is given in the Hanford 67-Series data volume (Nickola, 1977).

The lack of data during unstable atmospheric conditions does detract from the ultimate use of the curves of Figs. 6.4 to 6.9. For instance, the diffusion climatology at a given location can be put into a reasonable model and annual average concentrations can be predicted for a release from a specific stack height. If the less stable data were available from this study, the change in annual average concentration as a function of several changes in stack height could be estimated.

\subsection{CONCLUSIONS}

Figs. 6.4 to 6.9 present field measurements-based ratios of normalized crosswind-integrated concentration resulting from simultaneous release of tracers from two elevations. To a distance of $10 \mathrm{~km}$, these ratios are higher (implying lower benefits from increased stack height) than are ratios based on a Gaussian plume model with atmospheric stabilities as prescribed in NRC Regulatory Guide 1.23.

At distances relatively far from the source, the benefits in reduced concentration resulting from increasing source height from $26 \mathrm{~m}$ to $56 \mathrm{~m}$ plateau at approximately the ratio of wind speeds at the $26 \mathrm{~m}$ and $56 \mathrm{~m}$ levels. Benefits accruing as a result of increasing from $56 \mathrm{~m}$ to $111 \mathrm{~m}$ exceed the source height wind speed ratio at all distances. Conversely, benefits for an increase from $2 \mathrm{~m}$ to $26 \mathrm{~m}$ are lacking at distances beyond about $4 \mathrm{~km}$. 


\section{COMPARISON OF RECOMMENDED MODEL TO NRC MODEL}

The preceding chapters have shown that current diffusion theories are supported by the data from high-quality diffusion experiments. This has led to the recommendation of a diffusion model which differs from that currently used by the NRC. In this chapter the recommended model and the NRC model are summarized, and then their predictions are each compared to the diffusion data in order to quantify the differences in their predictive capabilities.

\subsection{RECOMMENDED DIFFUSION MODEL}

For releases near ground level, the analys is reported in Chapters 4-6 supports the recommendation of a bivariate Gaussian diffusion model,

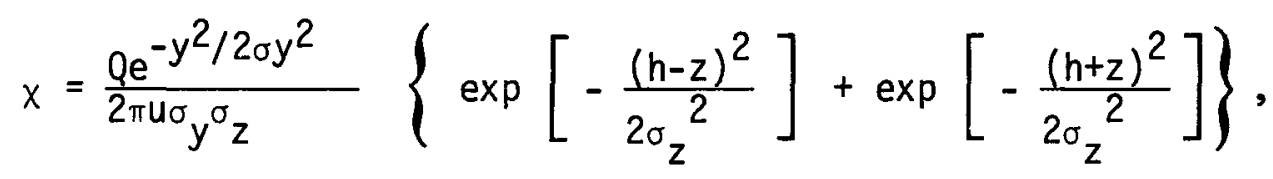

with $\sigma_{y}$ predictions based on Taylor's statistical theory and $\sigma_{z}$ and $u$ predictions based on Lagrangian similarity theory. $\sigma_{y}$ is calculated from the equation

$$
\sigma_{y}=S x \sigma_{\theta},
$$

with $S$ a function of downwind distance, averaging time for $\sigma_{\theta}$, and sampling (or release) time. Values of $S$ are recommended in Table 4.3 for sampling times of $30 \mathrm{~min}$ and $60 \mathrm{~min}$. $\sigma_{\theta}$ is to be measured near ground-level, e.g., $2 m-4 m$, with an averaging time of $5 \mathrm{sec}$.

The prediction of $\sigma_{z}$ from Lagrangian similarity theory is more complex, requiring the estimation of $z_{0}$ and $L$ from measurements of the wind and temperature profiles, and the computation of $\bar{z}$ from

$$
x=\frac{1}{k^{2}} \int_{z_{0}}^{\bar{z}}\left[f(c z / L)-f\left(c z_{0} / L\right)\right] \phi_{h}(z / L) d z .
$$

For the Gaussian formula (7.1), $c=0.66$ and $\sigma_{z}=\sqrt{\pi / 2} \bar{z}$. The functions $f$ and $\phi_{h}$ recommended by Businger et al. (1971) for use with $k=0.35$ are 
listed following Eq. (5.13). Comparable results were obtained with the functions recommended by Dyer (1974) for use with $k=0.41$.

Calculation of the Obukhov length $L$ requires values for the vertical fluxes of momentum and heat. These are estimated from profiles of wind and temperature, measured within the lowest $10 \mathrm{~m}$, by determining the best fit of the measurements to the empirical flux-gradient relationships of Businger et a1. (1971) or Dyer (1974). There are many techniques for doing this. In this study the Richardson number at height $z$ was calculated from the measured profiles and converted to $L$ using

$$
z / L=\phi_{m}^{2} R i / \phi_{h} \text {. }
$$

Nieuwstadt (1978) discusses several other techniques. The minimum data requirements are two levels of wind and temperature. The roughness length $z_{0}$ is then the height at which the wind speed extrapolates to zero. If $z_{0}$ is already known or visually estimated for the site, the wind need be measured at only one height. However since the calculation of $L$ (and $z_{0}$ ) is based on the gradient, the best estimates will follow from measurements at several levels. The estimation of $L$ from several different combinations of wind and temperature measurements is discussed in Chapter 3.

The wind speed $u$ is evaluated at the height $c \bar{z}$ or $0.53 \sigma_{z}$. The empirical wind profile $f$ may be used to interpolate or extrapolate the measured wind to other heights.

The preceding model has been shown to work best for ground-levei releases. For elevated releases, $\sigma_{\theta}$ at ground level is a good predictor of $\sigma_{y}$ only at long distances downwind of the source. Neither $\sigma_{\theta}$ at ground level nor $\sigma_{\theta}$ at release height is a good predictor close to the source. This investigation did not find that the available data on vertical diffusion from elevated sources could be explained by a generalized model. The best recommendation would be to use data from observations which most closely match the conditions for which the prediction is required. Several data sets are summarized in Sections 5.4, 5.5 and Chapter 6. 


\subsection{NRC DIFFUSION MODEL}

The standard NRC diffusion model is also based on the Gaussian plume, Eq. (7.1). The plume dimensions $\sigma_{y}$ and $\sigma_{z}$, however, are determined from the Pasquil1-Gifford-Turner curves as a function of downwind distance and stability class $(A-G)$. In the range 0.1 to $1 \mathrm{~km}$ these curves are based on diffusion observations over terrain with $z_{0} \simeq 3 \mathrm{~cm}$, and in the range 10-100 $\mathrm{km}$ they are appropriate for $z_{0} \simeq 30 \mathrm{~cm}$ (Pasquill, 1976). The sampling time for $\sigma_{y}$ is three minutes. For the model comparison of the following section, the Pasquill-Gifford-Turner $\sigma_{y}$ and $\sigma_{z}$ were calculated from power law formulas presented by Eimutis and Konicek (1972). For class $G, \sigma_{y}$ and $\sigma_{z}$ were calculated from

$$
\begin{aligned}
& \sigma_{y}(G)=0.67 \sigma_{y}(F) \\
& \sigma_{z}(G)=0.6 \sigma_{z}(F)
\end{aligned}
$$

U.S. NRC Regulatory Guide 1.23 (NRC, 1972) specifies two methods of determining the stability class. The $\Delta T / \Delta z$ method is based on the temperature difference between $40 \mathrm{~m}$ and $10 \mathrm{~m}$ for release heights at or below $40 \mathrm{~m}$ or the temperature difference between stack height and $10 \mathrm{~m}$ for higher releases. The $\sigma_{\theta}$ method is based on measurements of $\sigma_{\theta}$ for a period of $15 \mathrm{~min}$ to 1 hour, presumably at $10 \mathrm{~m}$ for releases below a height of $40 \mathrm{~m}$ and at stack height for elevated releases above $40 \mathrm{~m}$. Table 7.1 relates these meteorological parameters to NRC stability classes.

The wind speed $u$ is measured at a height of $10 \mathrm{~m}$ for releases below $40 \mathrm{~m}$ and at stack height for elevated releases above $40 \mathrm{~m}$.

\subsection{COMPARISON OF MODEL PREDICTIONS}

\subsubsection{Lateral Diffusion}

The recommended model for lateral dispersion specifies a particular relationship for the ratio $S=\sigma_{y} / x_{\theta}$ as a function of downwind distance, sampling time and averaging time. Different values of $S$ were therefore used for different test series. In this sense the formulation of the model may appear to be site specific since, in general, two sets of diffu- 
TABLE 7.1. NRC Classification of Atmospheric Stability

\begin{tabular}{|c|c|c|c|}
\hline $\begin{array}{c}\text { Stability } \\
\text { Classification }\end{array}$ & $\begin{array}{c}\text { Pasquill } \\
\text { Categories }\end{array}$ & $\sigma_{\theta}$ & $\begin{array}{l}\text { Temperature Chan } \\
\text { with Height }\left({ }^{\circ} \mathrm{C} / 1\right.\end{array}$ \\
\hline Extremely unstable & A & $25.0^{\circ}$ & $<-1.9$ \\
\hline Moderately unstable & B & $20.0^{\circ}$ & -1.9 to -1.7 \\
\hline Slightly unstable & c & $15.0^{\circ}$ & -1.7 to -1.5 \\
\hline Neutral & $D$ & $10.0^{\circ}$ & -1.5 to -0.5 \\
\hline Slightly stable & E & $5.0^{\circ}$ & -0.5 to 1.5 \\
\hline Moderately stable & $\mathrm{F}$ & $2.5^{\circ}$ & 1.5 to 4.0 \\
\hline Extremely stable & G & $1.7^{\circ}$ & $>4.0$ \\
\hline
\end{tabular}

sion data with similar values of $\tau$ and $t$ have not been recorded at two different sites. Thus, for example, the values of $S$ used for the Prairie Grass data, where $t=1 \mathrm{sec}$ and $\tau=600 \mathrm{sec}$, are different from those used for the NRTS data, where $t=5 \mathrm{sec}$ and $\tau=3600 \mathrm{sec}$.

The $\sigma_{y}$ predictions of the recommended model are shown in Fig. 7.1 for ground-level releases, plotted versus the measured values. As can be seen, the agreement is very good.

The NRC model uses either $\Delta T / \Delta z$ or $\sigma_{\theta}$ to select a stability class. Both methods have been used here for comparison. For the Prairie Grass data, detailed temperature profiles were measured between $25 \mathrm{~cm}$ and $16 \mathrm{~m}$. To obtain $T(40 \mathrm{~m})-T(10 \mathrm{~m})$, these were extrapolated to $40 \mathrm{~m}$ with secondorder polynomials in ln $z$. Suitable profiles were not available for the NRTS data. For the Green Glow and 30-Series, temperature measurements from a 400 foot tower were used to estimate $T(61 \mathrm{~m})-T(10 \mathrm{~m})$, and for the Hanford 67 data, direct measurements of $T(61 \mathrm{~m})-T(15 \mathrm{~m})$ were used.

The predictions of the NRC $\Delta T / \Delta Z$ model are shown in Fig. 7.2. As can be seen, the scatter of the data is considerably more than in Fig. 7.1. No particular bias is evident in the predicted values, so no systematic correction in the selection of stability classes would appear capable of producing significant improvements. 


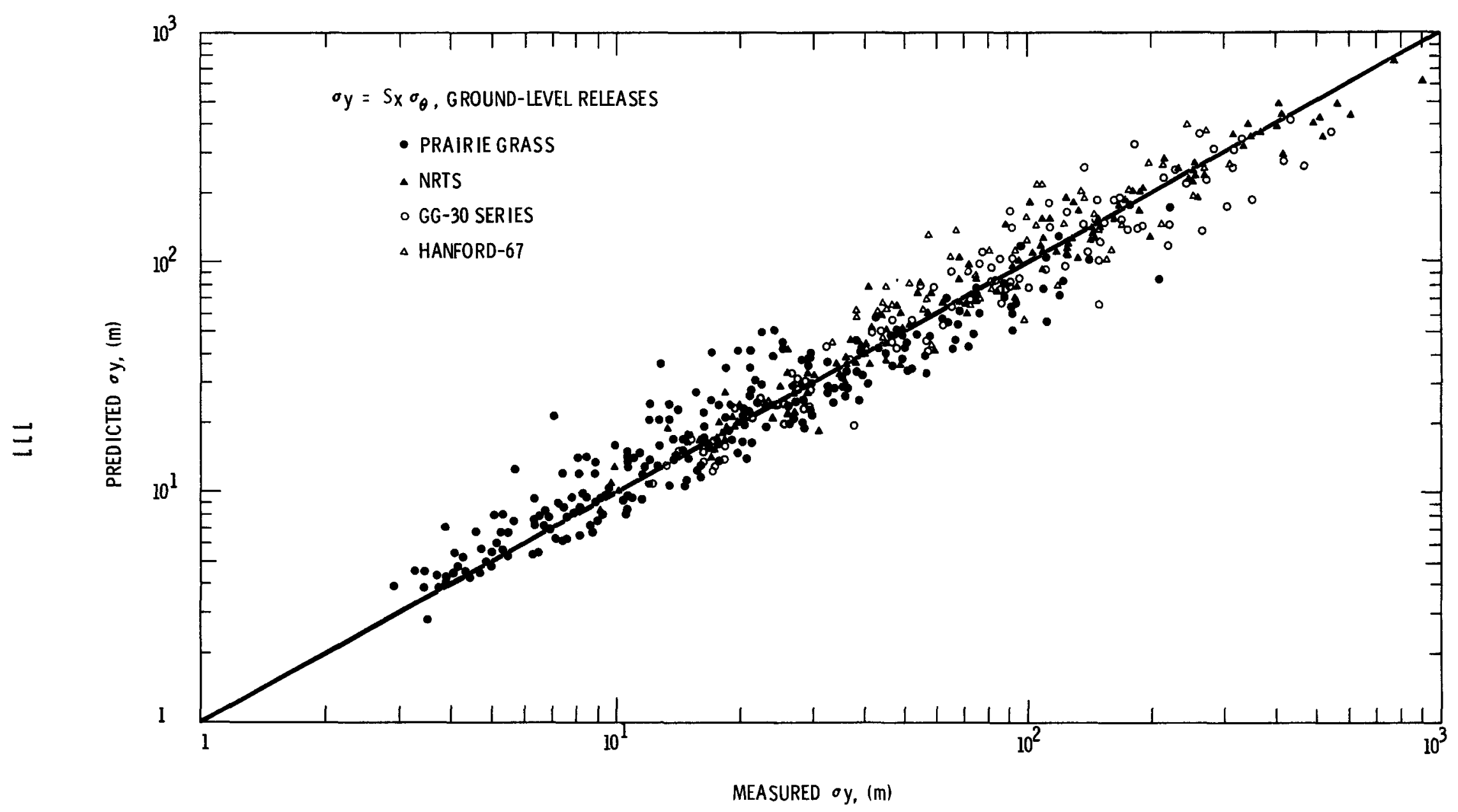

Figure 7.1. Predicted versus measured $\sigma_{y}$ for ground-level releases, $\sigma_{y}=S \times \sigma_{\theta}$. 


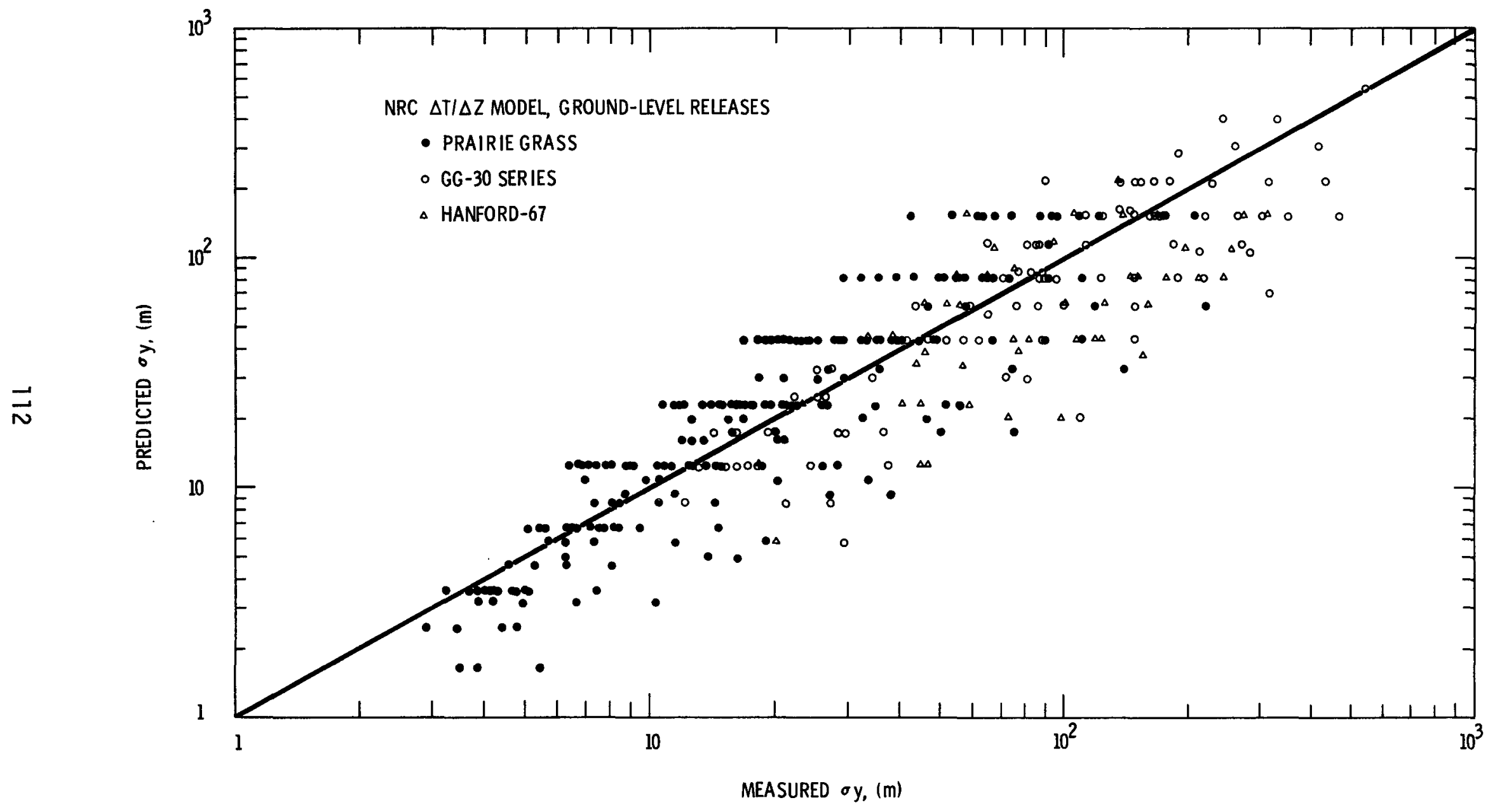

Figure 7.2. Predicted versus measured $\sigma_{y}$ for around-level releases, NRC $\Delta T / \Delta z$ model. 
The predictions of the NRC $\sigma_{\theta}$ model are shown in Fig. 7.3. For these ground-level releases, $\sigma_{\theta}$ was determined at a height of 2 to $4 \mathrm{~m}$ (see Chapter 3). Although the predictions are a bit more conservative, especially for the larger values of $\sigma_{y}$, the scatter of the NRC $\sigma_{\theta}$ model is not noticeably reduced from that of the $\Delta T / \Delta z$ model. This is surprising since the dependence of $\sigma_{y}$ on $\sigma_{\theta}$ has a sound physical basis, as has been shown in Chapter 4 . In that sense the NRC $\sigma_{\theta}$ model is similar to the recommended model; the latter, however, gives considerably better results.

For elevated releases, the limitations of using $\sigma_{\theta}$ as a predictor for $\sigma_{y}$ have been discussed in Section 4.3.1. The recommended model is most appropriate for ground-level releases. For the Hanford 67-Series $26 \mathrm{~m}$ releases (Figure 7.4), the scatter between predicted and measured values is larger than for the ground-level releases, while for the $56 \mathrm{~m}$ releases the scatter is greater yet. The NRC $\Delta T / \Delta Z$ predictions show far more scatter than the recommended model for the $26 \mathrm{~m}$ releases, and a comparable amount for the $56 \mathrm{~m}$ releases, although the NRC model predictions again tend to be conservative for both release heights.

The NRC $\sigma_{\theta}$ model can also be used for these elevated releases, and at least two choices can be made for the value of $\sigma_{\theta}$, i.e. at release height and near the ground. Fig. 7.5 shows results for the $26 \mathrm{~m}$ releases, with $\sigma_{\theta}$ at ground level as the predictor. Fig. 7.6 shows data from the same tests but with $\sigma_{\theta}$ measured near release height. For both the $26 \mathrm{~m}$ and $56 \mathrm{~m}$ releases, there is no apparent difference in the scatter produced by either choice. However, the release height values of $\sigma_{\theta}$ tend to be smaller for these tests, resulting in generally smaller estimates of $\sigma_{y}$.

\subsubsection{Vertical Diffusion}

The vertical diffusion models have been tested by comparing the predicted and measured values of the normalized CWIC, $x(x, z) / Q$, at ground level. Only two test series, Prairie Grass and NRTS, have been used for this comparison. The Green Glow-30 Series and Hanford 67 data have not been used because of the apparent deposition of the particulate tracers. 


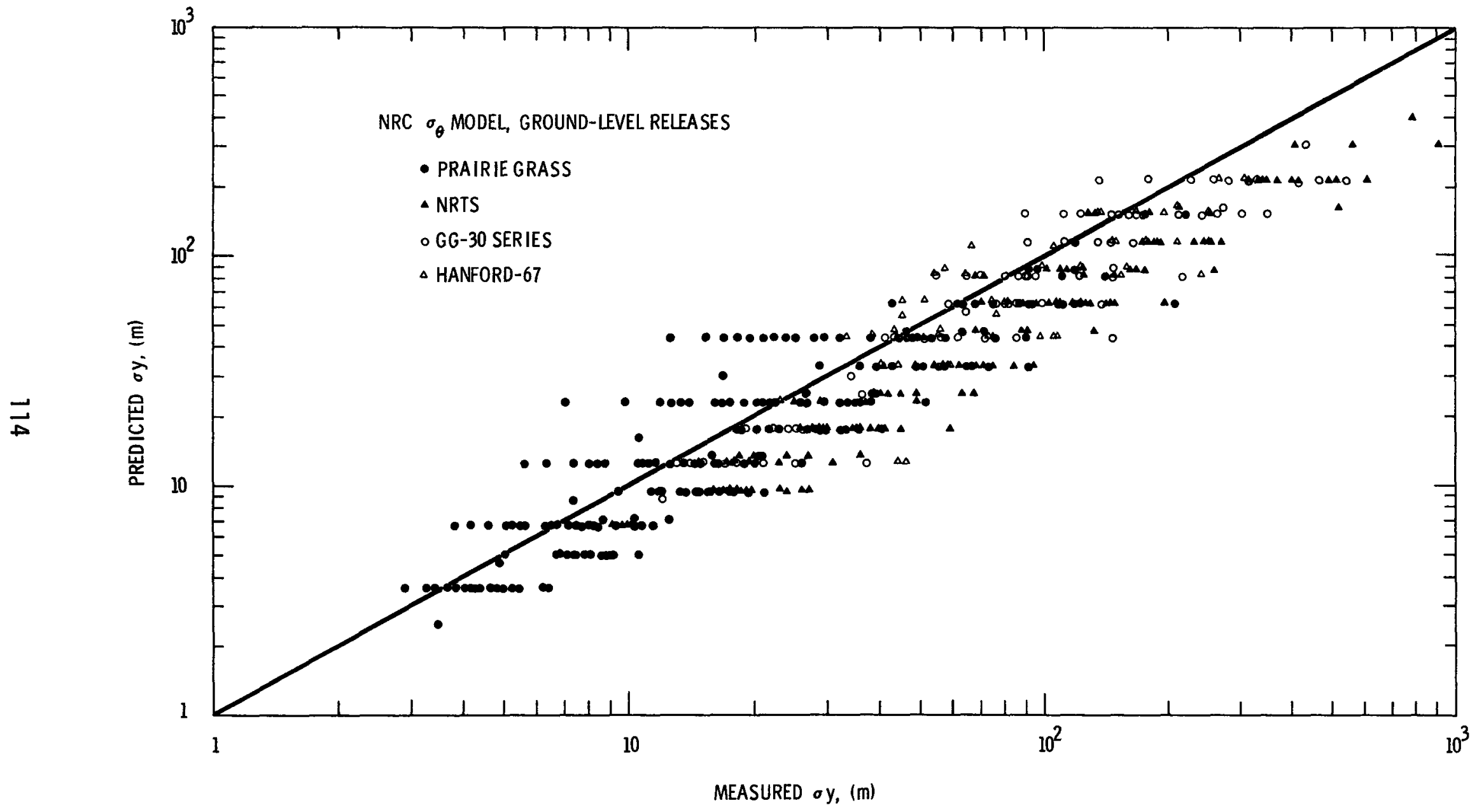

Figure 7.3. Predicted versus measured $\sigma_{y}$ for ground-level releases, NRC $\sigma_{\theta}$ model. 


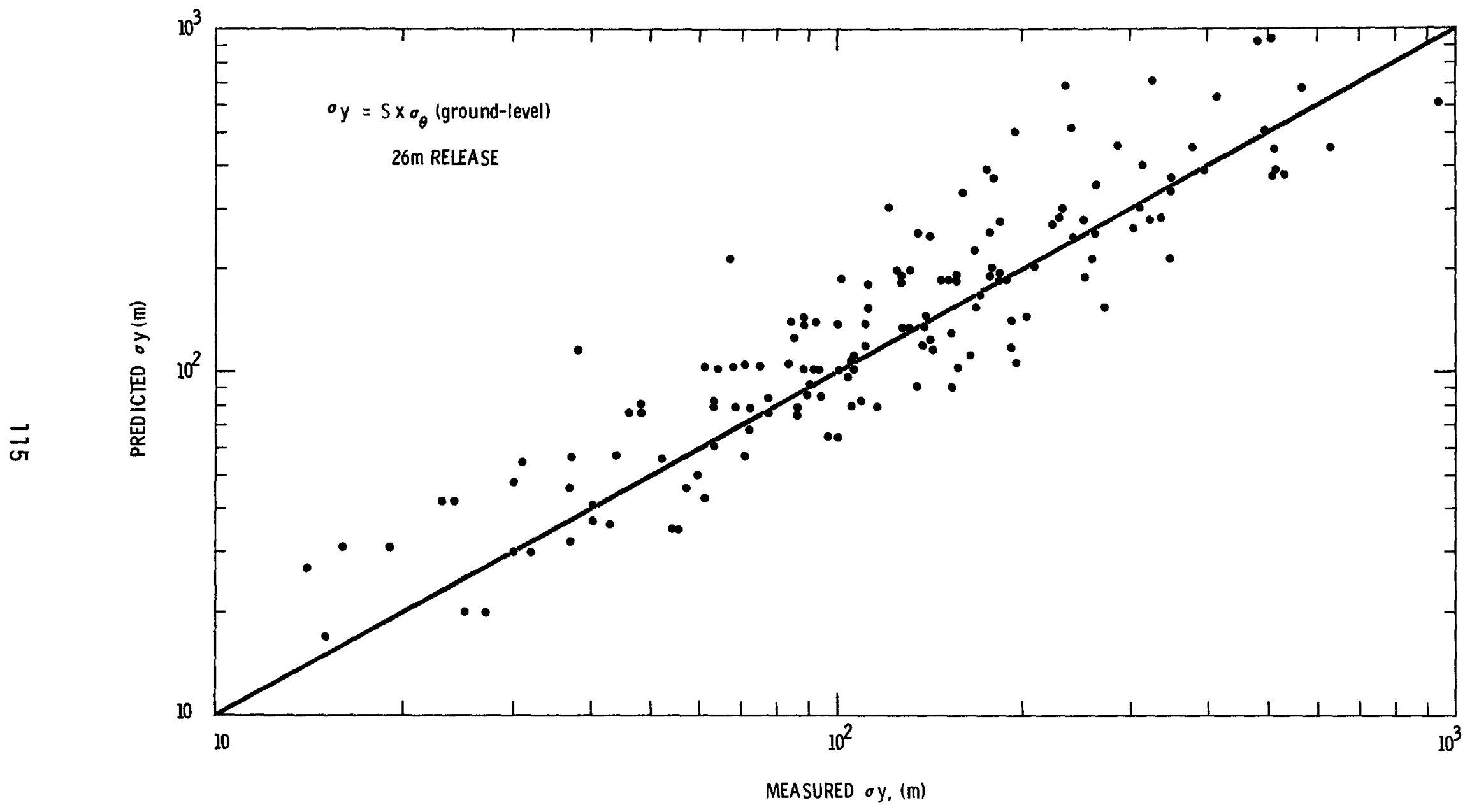

Figure 7.4. Prodicted versus measured $\sigma_{y}$ for $26 \mathrm{~m}$ releases, $\sigma_{y}=S \times \sigma_{\theta}$ (ground leve1). 


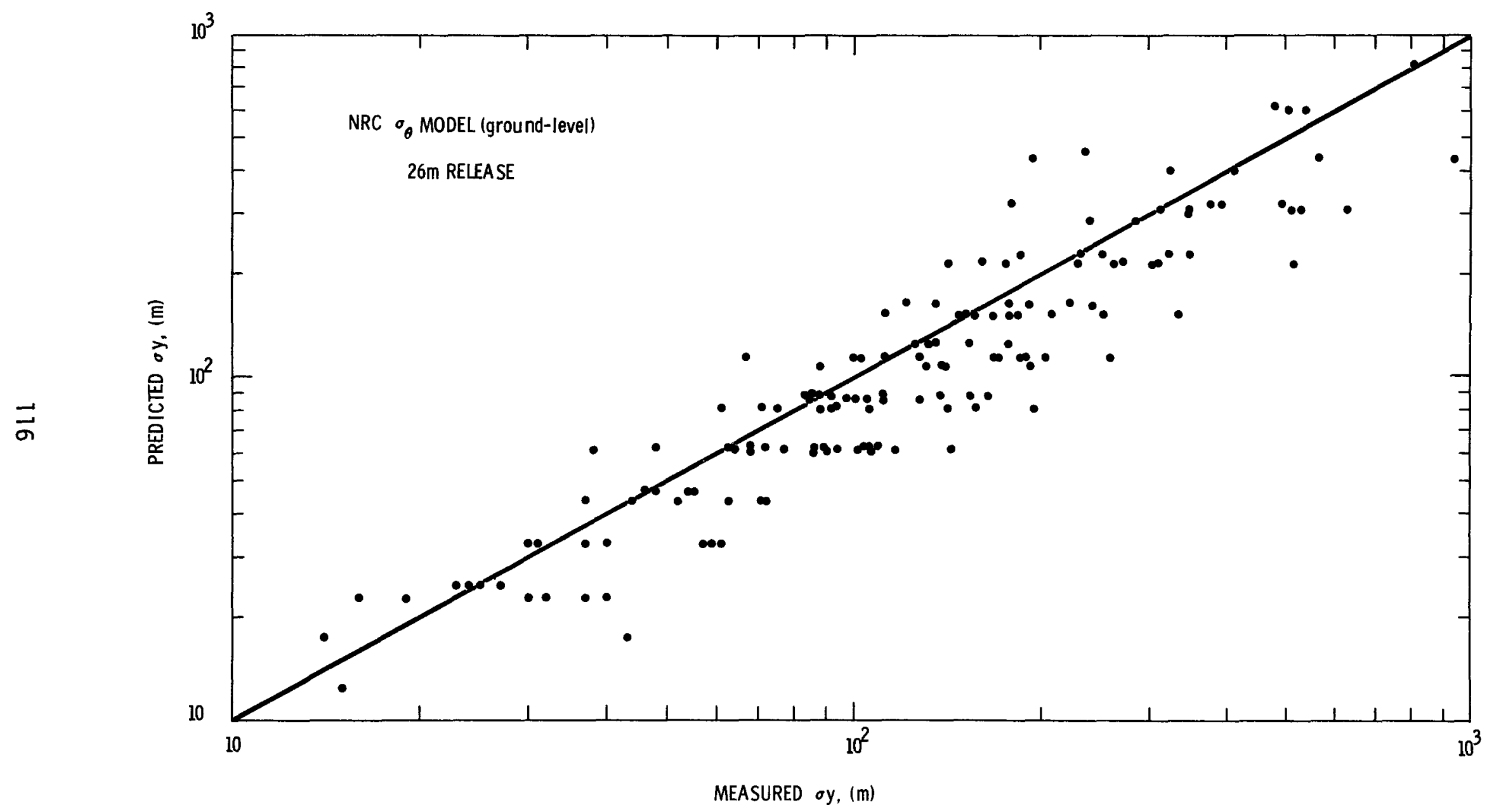

Figure 7.5. Predicted versus measured $\sigma_{y}$ for $26 \mathrm{~m}$ releases, MP.C $\sigma_{\theta}$ model (ground-level $\sigma_{\theta}$ ). 


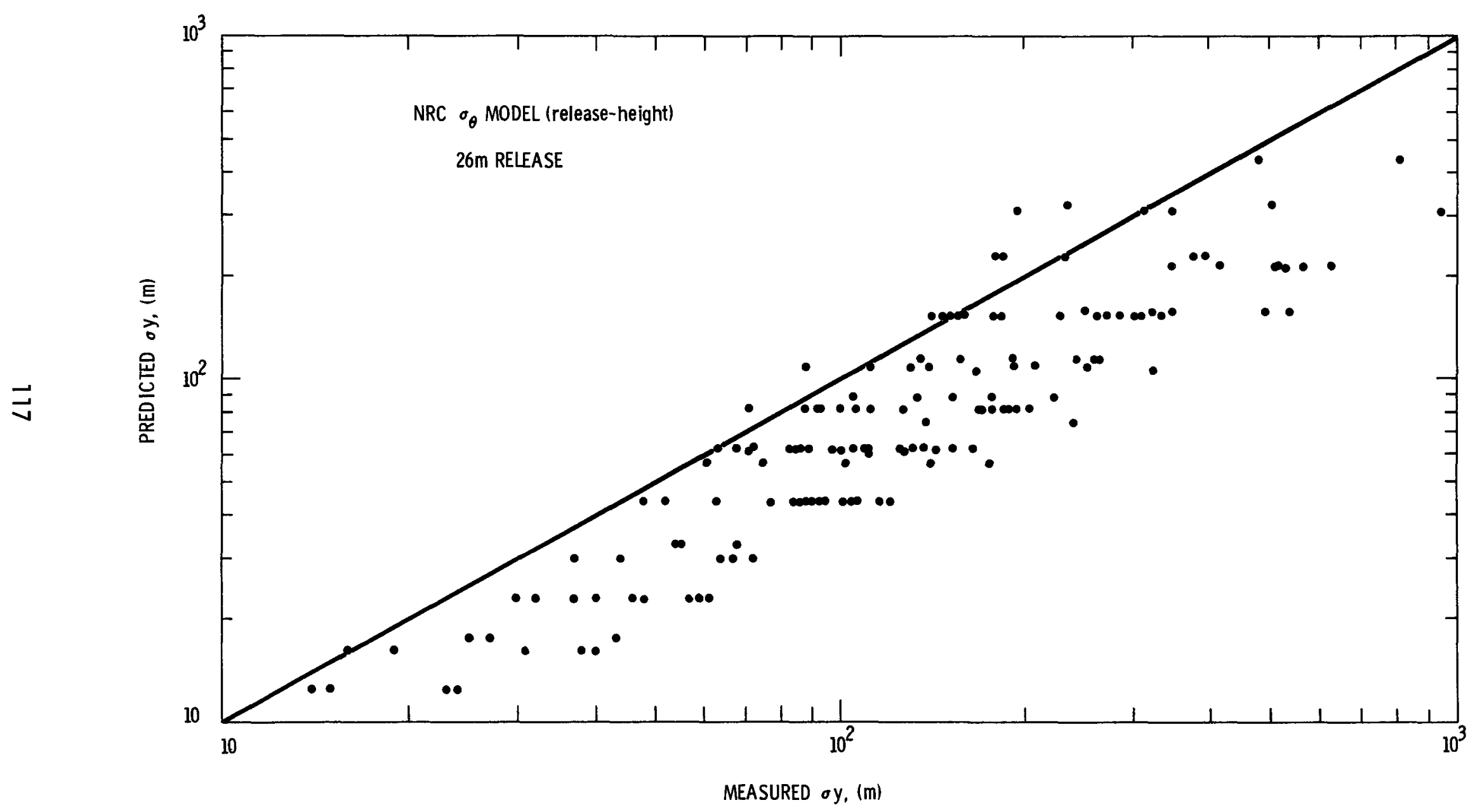

Figure 7.6. Predicted versus measured $\sigma_{y}$ for $26 \mathrm{~m}$ releases, NRC $\sigma_{\theta}$ model (release-height $\sigma_{\theta}$ ). 
The predictions of the recommended Lagrangian similarity model are shown in Fig. 5.7 for Prairie Grass. Fig. 7.7 shows the predictions of the NRC $\Delta T / \Delta Z$ model and Fig. 7.8 shows the predictions of the NRC $\sigma_{\theta}$ model. Both NRC models show considerably more scatter than the recommended model and both tend to underpredict Prairie Grass $x / Q$.

Much of the bias and scatter seen in Fig. 7.7 is caused by the use of $\Delta T / \Delta z$ to determine the stability class. Almost all of the unstable tests are classified as $A$ stability by the $\Delta T / \Delta Z$ method, and $\Delta T / \Delta Z$ is virtually uncorrelated with $1 / L$, the recommended stability parameter. Hence most unstable CWIC are greatly underpredicted. $\Delta T / \Delta Z$ is only slightly better correlated with $\mathrm{l} / \mathrm{L}$ on the stable side, and the $\Delta T / \Delta z$ method classifies most tests as being more stable than $1 / L$ would indicate according to Golder (1972). The resulting separation of stable and unstable tests may be seen in the values of $x / Q$ predicted for each distance in Fig. 7.7.

Similarly, $\sigma_{\theta}$ is poorly correlated with $1 / L$. The NRC $\sigma_{\theta}$ model classifies 3/4 of the Prairie Grass tests as $D$ and E stability. Consequently $x / Q$ is underpredicted for large $x / Q$ and is overpredicted for sma $11 \times / Q$. The use of $u(10 \mathrm{~m})$, rather than $u\left(0.53 \sigma_{z}\right)$, may also contribute to underprediction by the NRC models. For most of the data in Fig. 7.7 and $7.8,0.53 \sigma_{z}$ is less than $10 \mathrm{~m}$ and hence the NRC wind speed is too large. This is supported by the fact that the NRC predictions are worst at $50 \mathrm{~m}$ and improve with downwind distance, i.e. as $\sigma_{z}$ increases. The NRC $\Delta T / \Delta z$ predictions are best for stable conditions and the farthest arcs.

A third factor contributing to the underprediction is that the Pasquil1Gifford-Turner curves are appropriate for a $z_{0}$ of $3 \mathrm{~cm}$. Since the Prairie Grass site has a $z_{0}$ of $0.6 \mathrm{~cm}$, the P-G-T curves will overpredict $\sigma_{z}$ and underpredict $x / Q$.

Figs. 7.9 and 7.10 show the predictions of the recommended model and NRC $\sigma_{\theta}$ model for the NRTS data. Both models overpredict $x / Q$, the NRC model by about twice as much as the recommended model. The scatter of the recommended model is greater for the NRTS data than for the Prairie Grass data, but again the NRC model displays even more scatter. 


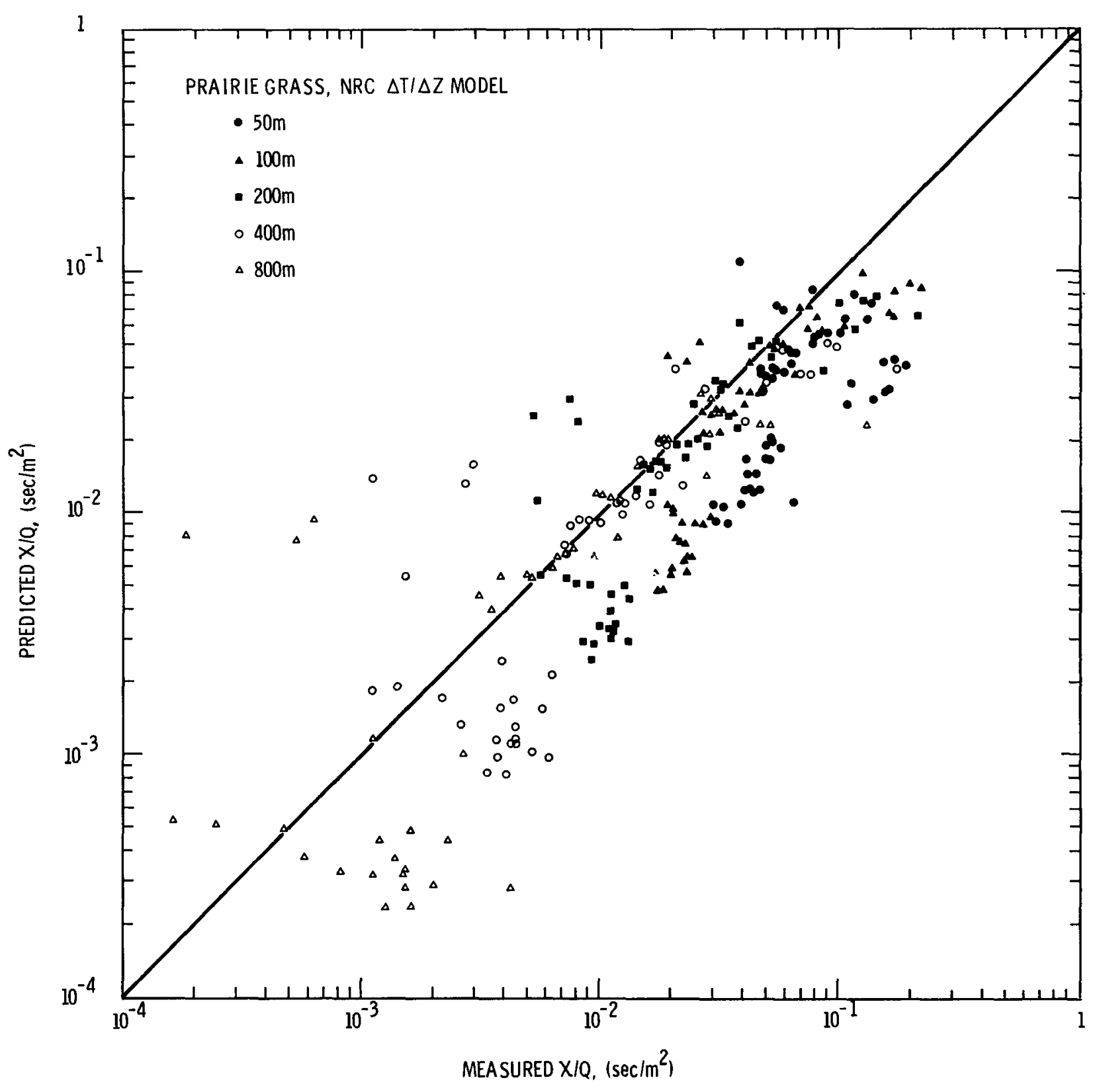

Figure 7.7. Predicted versus measured CWIC for Prairie Grass, NRC $\Delta T / \Delta z$ model. 


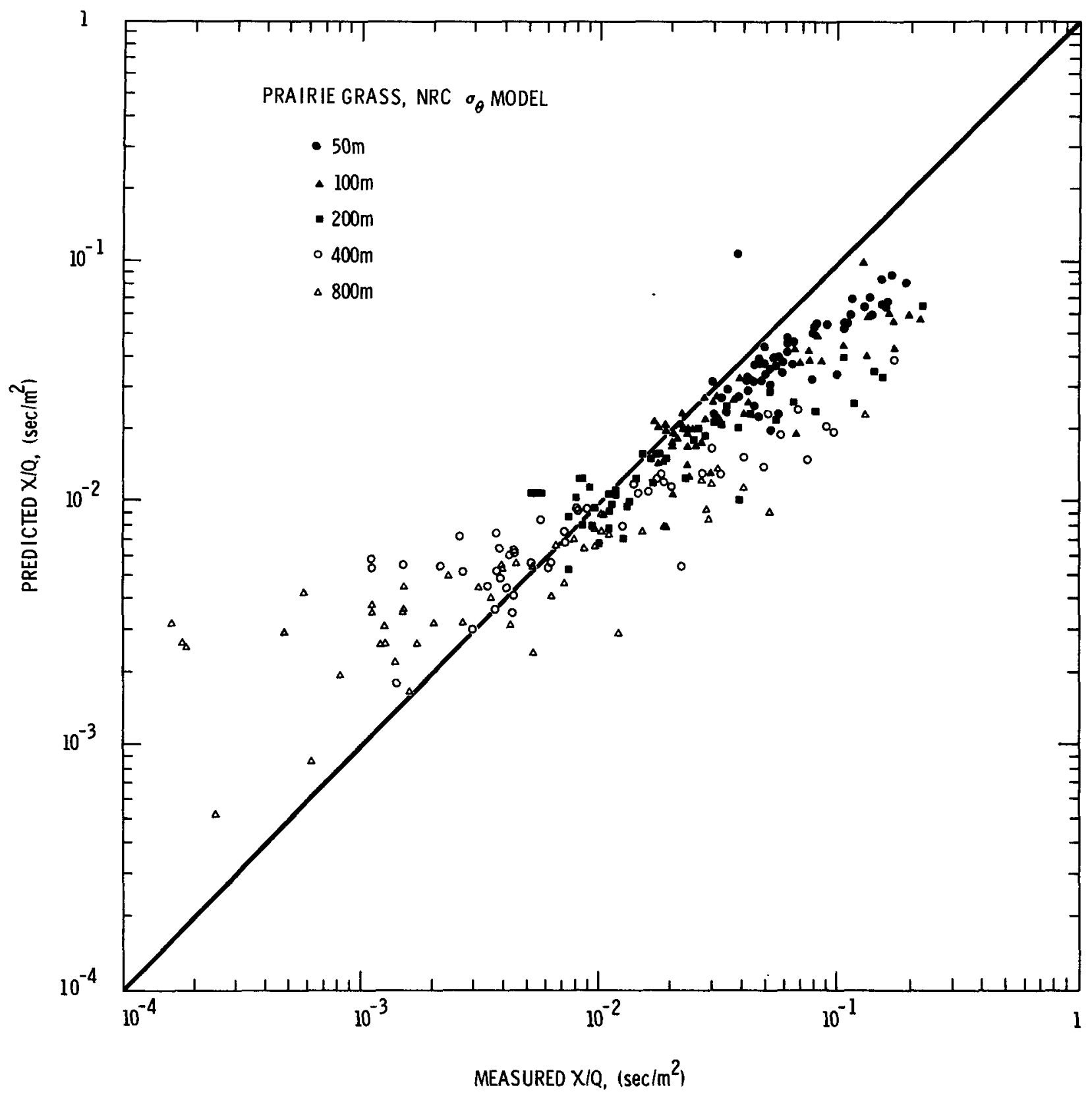

Figure 7.8. Predicted versus measured CWIC for Prairie Grass, NRC $\sigma_{\theta}$ model. 


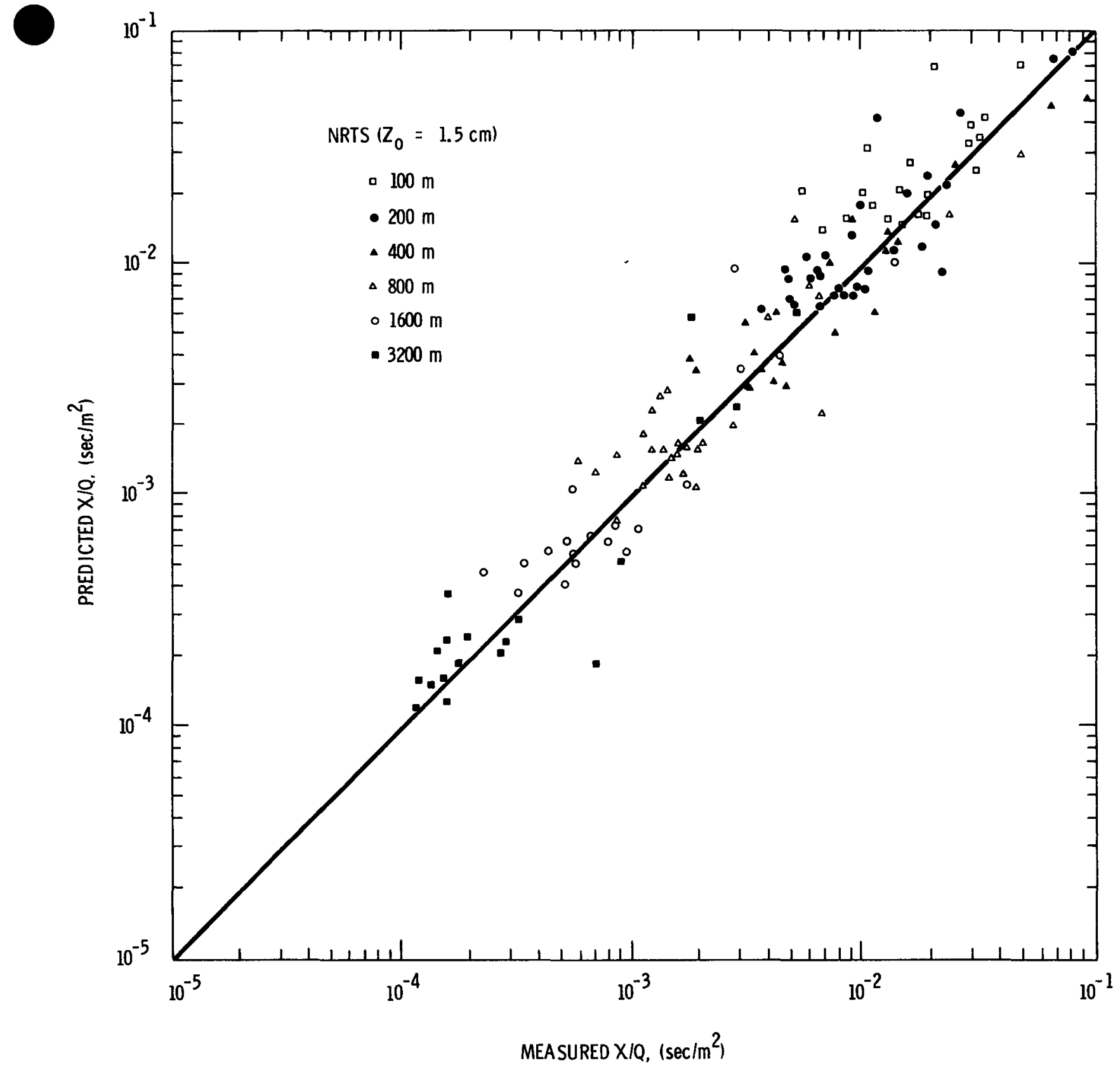

Figure 7.9. Predicted versus measured CWIC for NRTS, Lagrangian similarity model. 


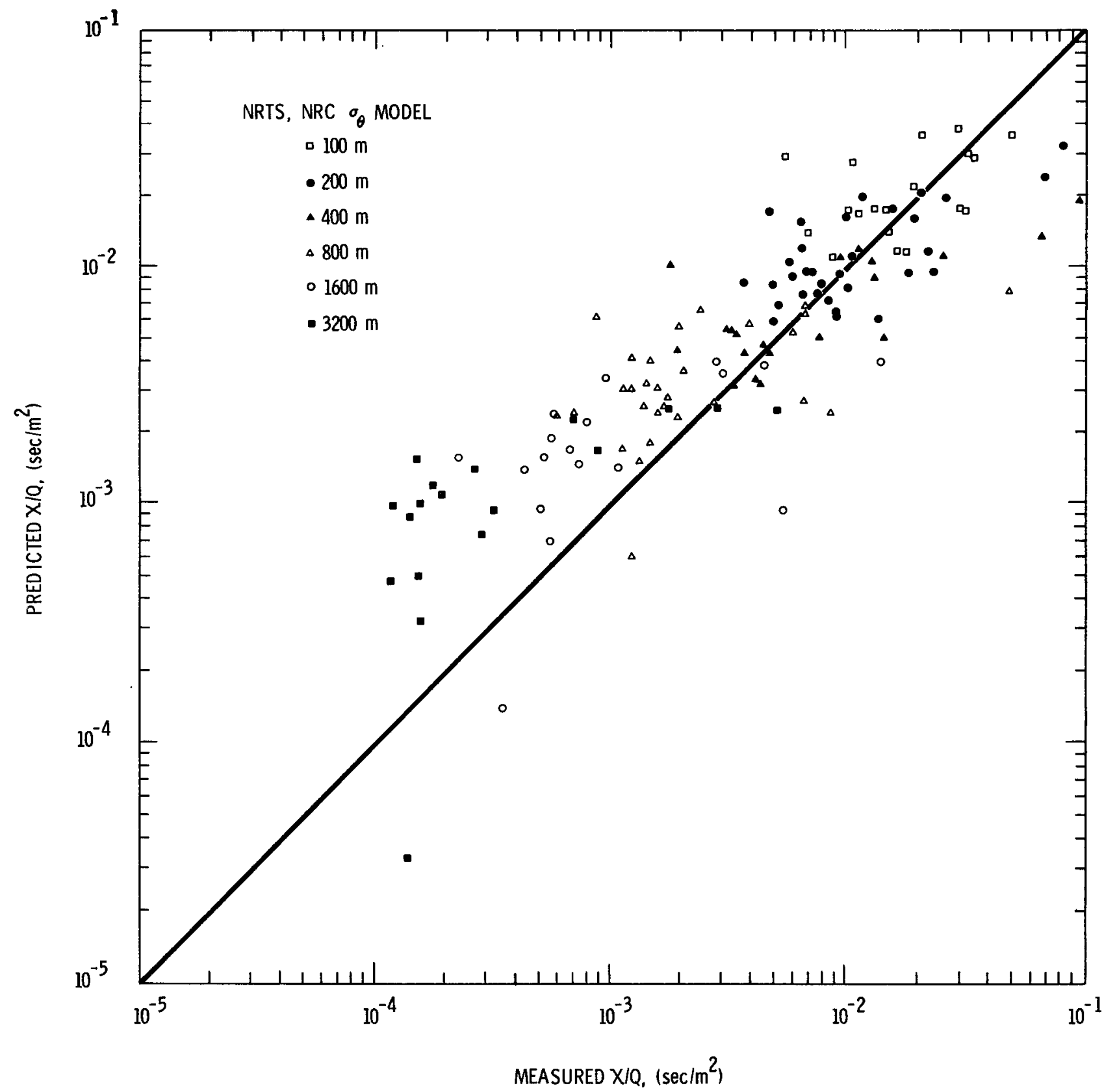

Figure 7.10. Predicted versus measured CWIC for NRTS, NRC $\sigma_{\theta}$ model. 
The NRC $\sigma_{\theta}$ model predictions in this case become worse with increasing distance. These overpredictions may again be partially caused by the use of $u(10 \mathrm{~m})$. For the NRTS data at $800 \mathrm{~m}$ to $3200 \mathrm{~m}, 0.53 \sigma_{z}$ is usualiy greater than $10 \mathrm{~m}$ and hence the NRC wind speed is too small.

\subsection{CONCLUSIONS}

A comparison of data from diffusion experiments to both the recommended diffusion model and the present NRC diffusion model demonstrates that improved predictions are possible with the recommended model, particularly for ground-level releases. In most cases the recommended diffusion model reduces both the scatter and the bias in the predicted values of $\sigma_{y}$ and CWIC.

The differences between measured values of $\sigma_{y}$ and CWIC and those predicted by the NRC models appear, in many cases, to be caused by the use of $\sigma_{\theta}$ and $\Delta T / \Delta z$ to determine a stability class. The NRC $\sigma_{\theta}$ and $\Delta T / \Delta z$ stability classes are poorly correlated with $1 / \mathrm{L}$, as well as with each other. The prediction of $\sigma_{y}$ is improved in the recommended model by basing its prediction directly on $\sigma_{\theta}$, without reference to a stability class, and by accounting for differences in sampling and averaging time among the sets of diffusion data. The prediction of ground-level CWIC is improved by using $1 / \mathrm{L}$, and hence both the wind and temperature gradients, to specify the atmospheric stability, by accounting for the effects of surface roughness on $\sigma_{z}$, and by specifying the advecting wind at a height of $0.53 \sigma_{z}$. 


\section{REFERENCES}

Barad, M. L., ed (1958), Project Prairie Grass, A Field Program in Diffusion, Geophysical Research Paper No. 59, Vols. I and II, AFCRC-TR-58-235, Air Force Cambridge Research Center, Bedford, MA.

Barad, M. L. and J. J. Fuquay, eds (1962), The Green Glow Diffusion Program, Geophysical Research Papers No. 73, Vols. I and II, Hanford Doc. Nos. HW71400 (I) and (II), AFCRL Doc. Nos. FACRL-62-251 (I) and (II), Hanford Laboratories, Richland, WA and Air Force Cambridge Research Laboratories, Bedford, MA.

Businger, J. A., J. C. Wyngaard, Y. Izumi and E. F. Bradely (1971), FluxProfile Relationships in the Atmospheric Surface Layer, J. Atmos. Sci. 28, 181-189.

Carpenter, S. B., J. M. Leavitt, F. W. Thomas, J. A. Frizzola and M. E. Smith (1968), Full-Scale Study of Plume Rise at Large Coal-Fired Electric Generating Stations, J. Air Pol1. Control Assoc., 18, 458-465.

Chatwin, P. C., (1968), The Dispersion of a Puff of Passive Substance in the Constant Stress Region, Quart. J. Roy. Meteor. Soc., 94, 401-411.

Chaudhry, F. H. and R. N. Meroney (1973), Similarity Theory of Diffusion and the Observed Vertical Spread in the Diabatic Surface Layer, Bound. Layer Meteor. 3 , 405-415.

Crawford, T. V. (1965), Moisture Transfer in Free and Forced Convection, Quart. J. Roy. Meteor. Soc., 91, 18-27.

Deardorff, J. W. and G. E. Willis (1975), A Parameterization of Diffusion into the Mixed Layer, J. Appl. Meteor. , 14, 1451-1458.

Draxier, R. R. (1976), Determination of Atmospheric Diffusion Parameters, Atmos. Envir., 10, 99-105.

Dyer, A. J. (1974), A Review of Flux Profile Relationships, Bound. Layer Meteor. , 7, 363-372.

Dyer, A. J. and B. B. Hicks (1970), Flux-Gradient Relationships in the Constant Flux Layer, Quart. J. Roy. Meteor. Soc., 96, 715-721.

Eimutis, E. C. and M. G. Konicek (1972), Derivations of Continuous Functions for the Lateral and Vertical Atmospheric Dispersion Coefficients, Atmos. Envir., 6, 859-863.

Elliot, W. P. (1961), The Vertical Diffusion of Gas from a Continuous Source, Int. J. Air Water Poll., 4, 33 . 
Fuquay, J. J., C. L. Simpson and W. T. Hinds (1964), Prediction of Environmental Exposures From Sources Near the Ground Based on Hanford Experimental Data, J.Appl. Meteor., 3, 761-770.

Galbally, I. E. (1971), Ozone Profiles and Ozone Fluxes in the Atmospheric Surface Layer, Quart. J. Roy. Meteor. Soc., 97, 18-29.

Gartrel1, F. E., F. W: Thomas, S. B. Carpenter, F. Pooler, B. B. Turner and J. M. Leavitt (1964), Full Scale Study of Dispersion of Stack Gases a Summary Report, Tennessee Val7ey Authority and U.S. Public Health Service PB1166679, Chattanooga, TN.

Gifford, F. A. (1976), Turbulent Diffusion-Typing Schemes: A Review, Nucl. Safety, 17, 68-86.

Golder, D. (1972), Relations Among Stability Parameters in the Surface Layer, Bound. Layer Meteor., 3, 47-58.

Harrington, Jr., J. B. (1965) Atmospheric Pollution of Aeroallergens; Meteorological Phase, Atmospheric Diffusion of Ragweed Pollen in Urban Areas: Vol. II, Text, (Vol. III, Tables), 06342-I-F, University of Michigan, College of Engineering, Dept. of Meteorology and Oceanography, Ann Arbor, MI.

Haugen,.D. A. and J. J. Fuquay, eds (1963), The Ocean Breeze and Dry Gulch Diffusion Programs, Vol. I, AFCRL-63-791(1), Hanford Doc. No. HW78435, Air Force Cambridge Research Labs., Bedford, MA and Hanford Laboratories, Richland, WA.

Haugen, D. A., ed (1972), Workshop on Micrometeorology, American Meteorological Society, Boston, MA.

Haugen, D. A., ed (1975), Lectures on Air Pollution and Environmental Impact Analyses, American Meteorological Society, Boston, MA.

Hay, J. S. and F. Pasquil1 (1959), Diffusion from a Continuous Source in Relation to the Spectrum and Scale of Turbulence, Advances in Geophysics, 6, 345-365.

Hess, C. D. and R. H. Clarke (1973), Time Spectra and Cross-Spectra of Kinetic Energy in the Planetary Boundary Layer, Quart. J. Roy. Meteor. Soc., 99, 130-153.

Hinds, W. T. and P. W. Nickola (1967), The Mountain Iron Diffusion Program: Phase I, South Vandenberg: Volume 1, BNWL-572, Vo T. I, Battelle Pacific Northwest Laboratories, Richland, WA. 
Hinds, W. T. and P. W. Nickola (1968), The Mountain Iron Diffusion Program: Phase I, South Vandenberg: Volume II, BNWL-572, Vol. II, Battelle Pacific Northwest Laboratories, Richland, WA.

Horst, T. W. (1977), A Surface Depletion Model for Deposition From a Gaussian Plume, Atmos. Envir., 11, 41-46.

Islitzer, N. A. (1961), Short-Range Atmospheric Dispersion Measurements From an Elevated Source, J. Meteor., 18, 443-450.

Islitzer, N. F. and R. K. Dumbauld (1963), Atmospheric DiffusionDeposition Studies Over Flat Terrain, Int. J. Air Wat. Poll., 7, 999-1022.

Kaimal, J. C., J. C. Wyngaard, Y. Izumi and 0. R. Cote' (1972), Spectral Characteristics of Surface-Layer Turbulence, Quart. J. Roy. Meteor. Soc., 98, 563-589.

Kaimal, J. C., J. C. Wyngaard, D. A. Haugen, 0. R. Coté, Y. Izumi, S. J. Caughey and C. J. Readings (1976), Turbulence Structure in the Convective Boundary Layer, J. App1. Met., 33, 2152-2169.

Lewellen, W. S. and M. E. Teske (1976), Second-Order Closure Modeling of Diffusion into the Atmospheric Boundary Layer, Bound. Layer Meteor., 10, 69-90.

Ludwick, J. D. (1966), Atmospheric Diffusion Studies with Fluorescein and Zinc Sulfide Particulates as Dual Tracers, J. Geophys. Res., 7l, 1553.

Maccready, Jr., P. B., T. B. Smith and M. A. Wolf (1961), Vertical Diffusion From a Low Altitude Line Source, Vols. I and II, Meteorology Research, Inc., ATtadena, CA.

Malhotra, R. C. and J. E. Cermak (1964), Mass Diffusion in Neutral and Unstably Stratified Boundary-Layer Flows, Int. J. Heat Mass Transfer, 7, $169-186$.

Miller, R. L., ed (1966), Victoria Diffusion Trials, Vols. I, II, IIb, MRI66 FR-374, Meteorology Research, Inc., A7tadena, CA.

Monin, A. S. and A. M. Yaglom (1965), Statistical Hydromechanics, Nauka Press, Moscow.

Nickola, P. W. (1965), Field Testing of a Fluorescein-Zinc Sulfide Dual Atmospheric Tracer Technique, BNWL-103, Battelle Northwest, Richland, WA. 
Nickola, P. W. (1977), The Hanford 67-Series: A Volume of Atmospheric Field Diffusion Measurements, PNL-2433, Battelle, Pacific Northwest Laboratories, Richland, WA. 99352.

Nieuwstadt, F. (1978), The Computation of the Friction Velocity $u_{\star}$ and the Temperature Scale $T_{\star}$ from Temperature and Wind Velocity Profîles by Least Square Methods, Bound. Layer Meteor., 10, 69-90.

Ogura, Y. (1959), Diffusion From a Continuous Source in Relation to a Finite Observation Interval, Advances in Geophysics, 6 , 149-159.

Panofsky, H. A. and I. Van der Hoven (1955), Spectra and Cross-Spectra of Velocity Componenets in the Mesometeorological Range, Quart. J. Roy. Meteor. Soc., 81, 603-606.

Pasqui11, F. (1971), Atmospheric Dispersion of Pollution, Quart J. Roy. Meteor. Soc., 97, 369-395.

Pasqui11, F. (1974), Atmospheric Diffusion, 2nd Ed., New York, John Wiley and Sons, $429 \mathrm{pp}$.

Pasquil1, F. (1975), Some Topics Relating to the Modeling of Dispersion in the Boundary Layer, EPA-650/4-75-015, U.S. Environmental Protection Agency, Research Triangle Park, NC.

Pasquill, F. (1976), Atmospheric Dispersion Parameters in Gaussian Plume Model ing Part II, Possible Requirements For Change in the Turner Workbook Values, EPA-600-4/76-030b, U.S. Environmenta] Protection Agency, Research Triangle Park, NC.

Paulson, C. A. (1970), The Mathematical Representation of Wind Speed and Temperature Profiles in the Unstable Atmospheric Surface Layer, J. Appl. Meteor., 9 , 857-861.

Shearer, D. L. and D. H. Minott (1976), Applicability of U.S. Army Test Data to Model Validation Needs of ERDA, Co0-2857-7, The Research Corp. of New England, Wethersfield, CT.

Simpson, C. L. (1961), Some Measurements of the Deposition of Matter and its Relation to Diffusion From a Continuous Point Source in a Stable Atmosphere, HW-69292-Rev, Hanford Atomic Products Operation, Richland, WA.

Sinclair, T. R., L. H. Allen, Jr. and E. R. Lemen (1975), An Analysis of Errors in the Calculation of Energy Flux Densities Above Vegetation by a Bowen-Ratio Profile Method, Bound. Layer Meteor., 8, 129-140.

Singer, I. A. and M. E. Smith (1966), Atmospheric Dispersion at Brookhaven National Laboratory, Int. J. Air Water Poll., 10, 125-135. 
Slade, D. H., ed (1968), Meteorology and Atomic Energy, TID-24190, U.S. Atomic Énergy Commission, Washington, D.C.

Smedman-Högström and U. Högström (1975), Spectral Gap in Surface Layer Measurements, J. Atmos. Sci., 32, 340-350.

Smith, T. B. and B. L. Niemann (1969), Shoreline Diffusion Program, Oceanside, California, Vols. I and II, MRI69 FR-860, Meteorology Research, Inc., Altadena, CA.

Taylor, J. H., ed (1965), Project Sandstorm, An Experimental Program in Atmospheric Diffusion, Environmental Research Papers, No. 134, AFCRL65-649, Air Force Cambridge Research Laboratories, Cambridge, MA.

Thomas, F. W., S. B. Carpenter and W. C. Colbaugh (1970), Plume Rise Estimates for Electric Generating Stations, J. Air Pol1. Control Assoc., 20, 170 .

Thomas, P. W., W. Hübschmann, L. A. König, H. Schüttelkopf, S. Vogt and M. Winter (1976a), Experimental Determination of the Atmospheric Dispersion Parameters Over Rough Terrain, Part 1, Measurements at the Karlsruhe Nuclear Research Center, KFK2285, Central Division for Nuclear Research, M. B. H. Karlsruhe, Federal Republic of Germany.

Thomas, P. and K. Nester (1976b), Experimental Determination of the Atmospheric Dispersion Parameters Over Rough Terrain, Part 2, Evaluation of Measurements, KFK 2286, Central Division for Nuclear Research, M. B. H. KarTsruhe, Federal Republic of Germany.

U.S. Nuclear Regulatory Commission (1972), On-Site Meteorological Programs, Regulatory Guide 1-23, USNRC, Washington, D.C.

Van der Hoven, I. (1976), A Survey of Field Measurements of Atmospheric Diffusion under Low-Wind-Speed Inversion Conditions, Nucl. Safety, 17, 2.

Vogt, K. J., H. Geiss, H. Nordsieck, G. Polster and F. Rohloff (1973), Investigations on the Propogation of Contaminated Air Trails in the Atmosphere, Jül-998-ST, Jülich Nuclear Research Institute, Jülich, Federat Republ ic of Germany.

Vogt, K. J. and H. Geiss (1974), Tracer Experiments on the Dispersion of Plumes Over Terrain of Major Surface Roughness, Jü7-1137-ST, Jülich Nuclear Research Institute, Jülich, Federal Republic of Germany.

Vogt, K. J. (1977a), A New System of Release Height Dependent Diffusion Parameters for the Gaussian Plume Model, Fourth International Clean Air Congress, May 16-20, 1977, Tokyo, Japan.

Vogt, K. J. (1977b), Empirical Investigations of the Diffusion of Waste Air Plumes in the Atmosphere, Nucl. Tech., 34, 43-57. 
Weber, A. H., K. R. McDonald and G. A. Briggs (1977), Turbulence Classification Schemes for Stable and Unstable Conditions, Joint Conference on Applications of Air Pollution Meteorology, Nov 29Dec 2, 1977, Salt Lake City, Utah, American Meteolological Society, Boston, MA. 
Appendix A

Computed Vertical Diffusion Data for Prairie Grass

\begin{tabular}{|c|c|c|c|c|c|c|c|}
\hline \multirow[b]{2}{*}{ Test } & \multirow{2}{*}{$\begin{array}{c}u_{(t}^{\star} \\
\mathrm{cm} / \mathrm{s})\end{array}$} & \multirow{2}{*}{$\stackrel{L}{(m)}$} & \multicolumn{5}{|c|}{ CWIC $/ Q\left(\mathrm{~s} / \mathrm{m}^{2}\right)$} \\
\hline & & & $50 \mathrm{~m}$ & $100 \mathrm{~m}$ & $200 \mathrm{~m}$ & $400 \mathrm{~m}$ & $800 m$ \\
\hline 1 & 17 & $-11^{*}$ & $8.59-2$ & $2.66-2$ & $6.24-3$ & $1.98-3$ & $7.69-4$ \\
\hline 2 & 12 & $-18 *$ & $8.81-2$ & $3.20-2$ & $7.83-3$ & $1.53-3$ & $9.65-4$ \\
\hline 4 & 6.6 & $5.1^{*}$ & $1.42-1$ & $2.60-1$ & $2.29-1$ & $2.23-1$ & $3.77-2$ \\
\hline 5 & 37 & -29 & $4.36-2$ & $2.26-2$ & $1.04-2$ & $3.78-3$ & $1.18-3$ \\
\hline 6 & 42 & -86 & $4.37-2$ & $2.46-2$ & $1.13-2$ & $4.42-3$ & $2.00-3$ \\
\hline 7 & 29 & -8.1 & $5.18-2$ & $2.02-2$ & $7.24-3$ & $1.41-3$ & $2.44-4$ \\
\hline 8 & 29 & -19 & $5.59-2$ & $2.87-2$ & $1.25-2$ & $4.28-3$ & $1.59-3$ \\
\hline 9 & 43 & -34 & $4.01-2$ & $2.25-2$ & $1.08-2$ & $4.42-3$ & $1.48-3$ \\
\hline 10 & 31 & -8.4 & $4.90-2$ & $1.98-2$ & $7.73-3$ & $2.16-3$ & $4.72-4$ \\
\hline $11^{-}$ & 47 & -68 & $3.72-2$ & $2.15-2$ & $1.19-2$ & $5.10-3$ & $1.89-3$ \\
\hline 12 & 50 & -48 & $3.39-2$ & $1.96-2$ & $1.09-2$ & $4.78-3$ & $2.08-3$ \\
\hline 13 & 6.6 & $5.8^{*}$ & $3.76-2$ & $1.25-1$ & $2.22-1$ & $1.73-1$ & $1.32-1$ \\
\hline 14 & 8.2 & $7.6^{*}$ & $1.53-1$ & $2.18-1$ & $1.52-1$ & $9.56-2$ & $2.93-2$ \\
\hline 15 & 22 & -6.6 & $7.39-2$ & $3.54-2$ & $1.41-2$ & $3.91-3$ & $1.19-3$ \\
\hline 16 & 23 & -3.3 & $5.43-2$ & $1.89-2$ & $5.18-3$ & $1.11-3$ & $1.83-4$ \\
\hline 17 & 20 & $50 *$ & $1.05-1$ & $6.44-2$ & $3.39-2$ & $1.76-2$ & $1.02-2$ \\
\hline 18 & 19 & $32 *$ & $1.08-1$ & $7.30-2$ & $4.53-2$ & $2.72-2$ & $1.85-2$ \\
\hline 19 & 37 & $-25^{\star}$ & $4.46-2$ & $2.19-2$ & $8.41-3$ & $2.59-3$ & $5.73-4$ \\
\hline 20 & 58 & -44 & $3.41-2$ & $1.78-2$ & $8.42-3$ & $3.37-3$ & $1.26-3$ \\
\hline 21 & 36 & 147 & $5.79-2$ & $3.57-2$ & $1.87-2$ & $9.81-3$ & $5.21-3$ \\
\hline 22 & 44 & 192 & $4.72-2$ & $2.71-2$ & $1.42-2$ & $7.08-3$ & $3.08-3$ \\
\hline 23 & 37 & 192 & $4.73-2$ & $2.89-2$ & $1.61-2$ & $7.30-3$ & $3.93-3$ \\
\hline 24 & 36 & 217 & $4.66-2$ & $2.73-2$ & $1.51-2$ & $8.13-3$ & $3.85-3$ \\
\hline
\end{tabular}


Appendix A continued

\begin{tabular}{|c|c|c|c|c|c|c|c|}
\hline \multirow[b]{2}{*}{ Test } & \multirow{2}{*}{$\underset{(\mathrm{cm} / \mathrm{s})}{u_{*}^{*}}$} & \multirow{2}{*}{$\underset{(m)}{L}$} & \multicolumn{5}{|c|}{$\mathrm{CWIC} / \mathrm{Q}\left(\mathrm{s} / \mathrm{m}^{2}\right)$} \\
\hline & & & $50 \mathrm{~m}$ & $100 \mathrm{~m}$ & $200 m$ & $400 m$ & $800 m$ \\
\hline 25 & 19 & -5.4 & $7.76-2$ & $2.62-2$ & $7.34-3$ & $2.92-3$ & $6.22-4$ \\
\hline 26 & 41 & -28 & $3.97-2$ & $2.26-2$ & $1.07-2$ & $4.04-3$ & $1.30-3$ \\
\hline 27 & 39 & -32 & $4.35-2$ & $2.36-2$ & $1.17-2$ & $4.61-3$ & $1.79-3$ \\
\hline 28 & 15 & 36 & $1.35-1$ & $7.92-2$ & $5.14-2$ & $2.96-2$ & $1.44-2$ \\
\hline 29 & 22 & $31^{\star}$ & $9.97-2$ & $6.50-2$ & $3.77-2$ & $2.21-2$ & $1.19-2$ \\
\hline 30 & 44 & -39 & $4.37-2$ & $2.43-2$ & $1.16-2$ & $4.21-2$ & $1.12-3$ \\
\hline 31 & 49 & -56 & $3.87-2$ & $2.31-2$ & $1.29-2$ & $6.14-3$ & $4.20-3$ \\
\hline 32 & 13 & $12 *$ & $1.59-1$ & $1.68-1$ & $1.15-1$ & $7.44-2$ & $5.15-2$ \\
\hline 33 & 47 & -65 & $3.26-2$ & $1.97-2$ & $9.32-3$ & $3.69-3$ & $1.24-3$ \\
\hline 34 & 57 & -69 & $3.01-2$ & $1.75-2$ & $9.13-3$ & $4.08-2$ & $1.72-3$ \\
\hline $35 \mathrm{~S}$ & 23 & 54 & $8.80-2$ & $5.74-2$ & $3.19-2$ & $1.81-2$ & $9.60-3$ \\
\hline 35 & 7.8 & $5.2^{*}$ & $1.70-1$ & $1.95-1$ & $1.41-1$ & $8.83-2$ & $2.66-2$ \\
\hline 36 & 11 & $17 *$ & $1.93-1$ & $1.70-1$ & $9.97-2$ & $5.74-2$ & $3.97-2$ \\
\hline 37 & 28 & 93 & $6.07-2$ & $3.86-2$ & $2.07-2$ & $1.15-2$ & $6.63-3$ \\
\hline 38 & 26 & 86 & $7.76-2$ & $4.70-2$ & $2.53-2$ & 1. $38-2$ & $7.76-3$ \\
\hline 39 & 13 & $13 *$ & $1.12-1$ & $6.78-2$ & $3.82-2$ & $1.97-2$ & - \\
\hline 40 & 11 & $13 *$ & $1.15-1$ & $7.38-2$ & $4.29-2$ & - & $1.51-2$ \\
\hline 41 & 23 & 56 & $7.93-2$ & $5.41-2$ & $3.13-2$ & $1.86-2$ & $1.11-2$ \\
\hline 42 & 35 & 110 & $5.14-2$ & $3.06-2$ & $1.69-2$ & $8.85-3$ & $4.96-3$ \\
\hline 43 & 33 & -13 & $5.06-2$ & $2.47-2$ & $1.10-2$ & $3.79-3$ & $1.18-3$ \\
\hline 44 & 38 & -25 & $4.46-2$ & $2.30-2$ & $1.08-2$ & $4.36-3$ & $1.37-3$ \\
\hline 45 & 37 & -89 & $5.20-2$ & $3.09-2$ & $1.64-2$ & $7.21-3$ & $3.51-3$ \\
\hline 46 & 33 & 92 & $6.29-2$ & $3.96-2$ & $2.24-2$ & $1.23-3$ & $6.27-3$ \\
\hline
\end{tabular}


Appendix A continued

\begin{tabular}{|c|c|c|c|c|c|c|c|}
\hline \multirow[b]{2}{*}{ Test } & \multirow{2}{*}{$\underset{(\mathrm{cm} / \mathrm{s})}{u^{\star}}$} & \multirow{2}{*}{$\stackrel{L}{(m)}$} & \multicolumn{5}{|c|}{$\mathrm{CWIC} / \mathrm{Q}\left(\mathrm{s} / \mathrm{m}^{2}\right)$} \\
\hline & & & $50 \mathrm{~m}$ & $100 \mathrm{~m}$ & $200 m$ & $400 \mathrm{~m}$ & $800 m$ \\
\hline 47 & 22 & -5.3 & $5.77-2$ & $2.32-2$ & $7.80-3$ & $2.68-3$ & $5.32-4$ \\
\hline $48 S$ & 21 & -5.2 & $4.78-2$ & $1.74-2$ & $5.38-3$ & $1.49-3$ & $1.78-4$ \\
\hline 48 & 48 & -64 & $2.95-2$ & $1.68-2$ & $8.27-3$ & $3.73-3$ & $1.51-3$ \\
\hline 49 & 43 & -25 & $4.26-2$ & $2.31-2$ & $1.14-2$ & $4.37-3$ & $1.52-3$ \\
\hline 50 & 42 & -31 & $4.10-2$ & $2.20-2$ & $8.98-3$ & $3.79-3$ & $1.11-3$ \\
\hline 51 & 43 & -33 & $4.62-2$ & $2.40-2$ & $9.80-3$ & $3.66-3$ & $8.20-4$ \\
\hline 52 & 30 & -7.9 & $5.16-2$ & $1.88-2$ & $5.56-3$ & $1.10-3$ & $1.58-4$ \\
\hline 53 & 13 & $10 *$ & $1.54-1$ & $1.32-1$ & $8.32-2$ & $5.07-2$ & $3.10-2$ \\
\hline 54 & 23 & 35 & $8.08-2$ & $5.03-2$ & $2.97-2$ & $1.74-2$ & $1.02-2$ \\
\hline 55 & 35 & 112 & $5.31-2$ & $3.20-2$ & $1.76-2$ & $8.06-2$ & $4.42-3$ \\
\hline 56 & 28 & 65 & $6.08-2$ & $4.23-2$ & $2.32-2$ & $1.25-2$ & $7.09-3$ \\
\hline 57 & 44 & -189 & $4.15-2$ & $2.11-2$ & $1.32-2$ & $6.26-3$ & $2.64-3$ \\
\hline 58 & 14 & $15^{*}$ & $1.60-1$ & $1.62-1$ & $1.05-1$ & $6.79-2$ & $4.63-2$ \\
\hline 59 & 16 & $20 *$ & $1.40-1$ & $1.29-1$ & $7.95-2$ & $4.87-2$ & $2.86-2$ \\
\hline 60 & 27 & 50 & $6.12-2$ & $3.80-2$ & $2.27-2$ & $1.17-2$ & $7.02-3$ \\
\hline 61 & 49 & -33 & $3.46-2$ & $2.02-2$ & $1.12-2$ & $5.15-3$ & $1.98-3$ \\
\hline 62 & 32 & -27 & $4.92-2$ & $2.67-2$ & $1.87-2$ & $5.69-3$ & $2.31-3$ \\
\hline 65 & 27 & 54 & $6.21-2$ & $4.22-2$ & $2.44-2$ & $1.46-2$ & $8.59-3$ \\
\hline 66 & 19 & $32 *$ & $1.07-1$ & $8.33-2$ & $5.35-2$ & $3.22-2$ & $1.89-2$ \\
\hline 67 & 27 & 79 & $6.48-2$ & $4.55-2$ & $2.74-2$ & $1.61-2$ & $9.45-3$ \\
\hline 68 & 16 & $31^{*}$ & $1.30-1$ & $1.04-1$ & $6.34-2$ & $4.01-2$ & $2.80-2$ \\
\hline
\end{tabular}

* Computed $L$ varies by more than a factor of 2 between $1 \mathrm{~m}$ and $4 \mathrm{~m}$. 
-

- 


\section{GLOSSARY OF SYMBOLS}

a

b

CWIC

c

$c^{\prime}$

$c_{p}$

g

H

h

K

$k_{h}$

$k_{m}$

k

$F_{E}(n)$

$F_{L}(n)$

$\mathrm{F}$

f

$f_{\max }$

$f_{\min }$

L

n

$\mathrm{p}$
$=$ constant in Eq. 5.1

$=$ constant of vertical distribution, Eq. 5.7

$=$ Crosswind-integrated concentration of contaminant, $x(x, z)$

$=$ constant relating advection wind to $\bar{z}$, Eq. 5.2

$=\sqrt{2 / \pi} \mathrm{c}$ in Eq. 5.16

= specific heat of air at constant pressure

= acceleration due to gravity

= vertical heat flux

= source height

= eddy diffusivity of contaminant

= eddy diffusivity of heat

= eddy diffusivity of momentum

= von Karman constant

$=$ Eulerian spectral distribution of lateral wind component, Chapter 4

= Lagrangian spectral distribution of the lateral wind component

$=$ vertical distribution of contaminant, Eq. 5.6

$=n z / u$, dimensionless frequency, Chapter 4

$=$ dimensionless frequency at which the wind spectrum achieves its maximum value

$=$ dimensionless frequency at which spectral gap occurs and spectrum has a minimum value

$=$ obukhov length $\left[-u_{*}^{3} \rho c_{p} \theta /(\mathrm{kgH})\right]$

= frequency

$=$ coefficient of power law expression for $\sigma_{y}$ and $\sigma_{z}$, Eq. 5.18 
$=$ rate of contaminant emission

$=$ Exponent of power law expression for $\sigma_{y}$ and $\sigma_{z}$, Eq. 5.18

$=\operatorname{Richardson}$ number $\left[g(\partial \theta / \partial z) / \theta(\partial u / \partial z)^{2}\right]$

= exponent of vertical distribution, Eq. 5.7

$=\sigma_{y} / x \sigma_{\theta}$

$=$ temperature

$=$ travel time $[\simeq x / u]$

$=$ time

= averaging time for meteorological measurements

$=$ Lagrangian integral time scale

$=$ wind speed

= friction velocity $\left[(\tau / \rho)^{1 / 2}\right]$

$=$ downwind coordinate

$=$ mean downwind distance of contaminant

= crosswind or lateral coordinate

= vertical coordinate

$=$ mean height of contaminant, Eq. 5.8

$=$ roughness length

= ratio of Lagrangian to Eulerian time scales

$=$ gamma function, $\Gamma(z)=\int_{0}^{\infty} t^{z-1} 0^{-t} d t$

= a difference between measurements at two heights, e.g. $\Delta T$

$=$ potential temperature

$=$ density of air

$=$ standard deviation of the horizontal, crosswind distribution of contaminant

$=$ standard deviation of the vertical distribution of contaminant 


\begin{tabular}{|c|c|c|}
\hline$\sigma_{v}$ & $=$ & $\begin{array}{l}\text { standard deviation of the fluctuations of the } \\
\text { lateral wind component }\end{array}$ \\
\hline$\sigma_{\theta}$ & $=$ & $\begin{array}{l}\text { standard deviation of the fluctuations of the } \\
\text { horizontal wind direction }\end{array}$ \\
\hline$\sigma_{\phi}$ & $=$ & $\begin{array}{l}\text { standard deviation of the fluctuations of the } \\
\text { vertical wind direction }\end{array}$ \\
\hline$\tau$ & $=$ & sampling time or duration of tracer release \\
\hline$\tau$ & $=$ & shear stress, see definition of $u_{\star}$ \\
\hline$\Phi$ & $=$ & diabatic function in Eq. 5.1 \\
\hline$\phi$ & $=$ & diabatic function in Eq. 5.4 \\
\hline$\phi_{h}$ & $=$ & $\begin{array}{l}\text { dimensionless potential temperature gradient } \\
{\left[u_{*} k z\left(\rho c_{p} / H\right) \partial \theta / \partial z\right]}\end{array}$ \\
\hline$\phi_{\mathrm{m}}$ & $=$ & dimensionless wind-shear $\left[\left(k z / u_{*}\right) \partial u / \partial z\right]$ \\
\hline$x(x, y, z)$ & $=$ & contaminant concentration \\
\hline$x(x, z)$ & $=$ & $\begin{array}{l}\text { crosswind-integrated contaminant concentra } \\
\text { Eq. } 5.17\end{array}$ \\
\hline
\end{tabular}

